\title{
Donor-and-acceptor substituted truxenes as multi-functional fluorescent probes
}

\author{
Mao-Sen Yuan, Zhi-Qiang Liu, and Qi Fang*
}

State Key Laboratory of Crystal Materials, Shandong University, 250100 Jinan, P.R. China

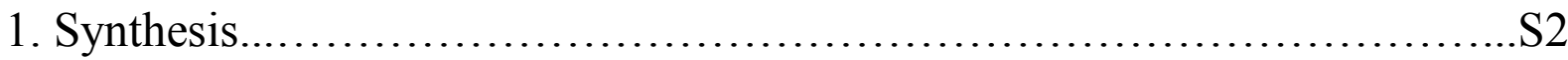

2. Absorption and emission graphics................................. S7

3. Absorption and emission data (Table 3)..............................S10

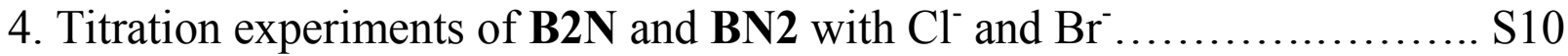

5. Calculation of the association constants of BN2 and B2N with $\mathrm{F}^{-} \ldots \ldots \ldots . . . \mathrm{S} 11$

6. Computational work and the results.................................. 15

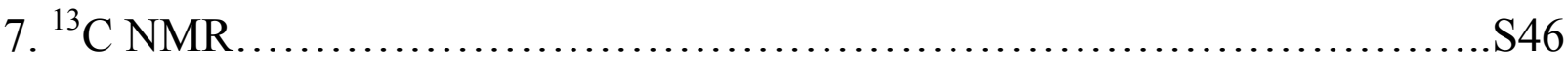




\section{Synthesis}

\subsection{Synthesis of 1-3, 5-7 as precursors of N1, B1, N2, B2, BN2 and B2N}

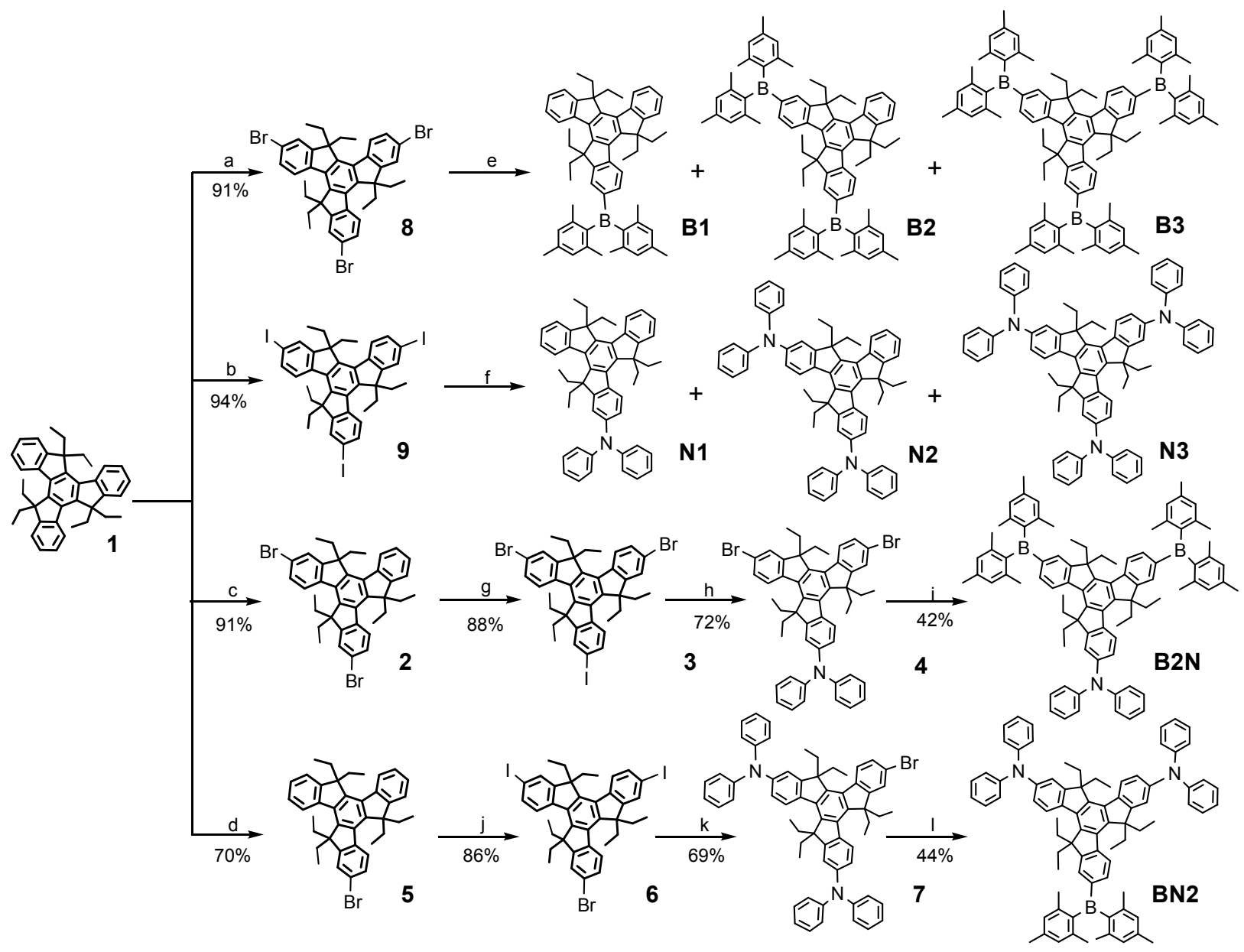

Synthesis of 2-Dimesitylboryl-5,5,10,10',15,15'-hexaethyltruxene (B1) and 2,7-Di(dimesitylboryl)-5,5',10,10',15,15'-hexaethyltruxene (B2). $n$-BuLi (2.8 M solution in $n$-hexane, $4.50 \mathrm{~mL}, 12.6 \mathrm{mmol})$ was added to a stirred solution of compound $\mathbf{8}(1.10 \mathrm{~g}, 1.48$ $\mathrm{mmol})$ in THF $(30 \mathrm{~mL})$ under nitrogen at $-78^{\circ} \mathrm{C}$ over $20 \mathrm{~min}$, and followed by warming to room temperature naturally. After reacting for further $4 \mathrm{~h}$, the reactants were cooled to $-78{ }^{\circ} \mathrm{C}$ again, and dimesitylboron fluoride $(2.20 \mathrm{~g}, 8.2 \mathrm{mmol})$ in THF $(15 \mathrm{~mL})$ was injected over $30 \mathrm{~min}$. The temperature was allowed to naturally rise to room temperature and the mixture continuously stirred for two days. Then the reactants were diluted with ethyl acetate, washed with water and dried over magnesium sulfate. After removing solvents, the crude product was obtained. After 
purified by column chromatography on silica gel, eluting with dichloromethane-petrol ether (1: 20), compounds B1 (0.13 g, 12\%, $\left.R_{f}=0.42\right)$, B2 (0.25 g, 17\%, $\left.R_{f}=0.35\right)$, and B3 (0.45 g, $24 \%$, $\left.R_{f}=0.18\right)$ were obtained. B1: a white powder, mp: $>400{ }^{\circ} \mathrm{C} .{ }^{1} \mathrm{H} \mathrm{NMR}\left(\mathrm{CDCl}_{3}, 400 \mathrm{MHz}, \mathrm{ppm}\right)$ $\delta$ 0.15-0.17 (m, 18H), $2.00(\mathrm{~s}, 18 \mathrm{H}), 2.27(\mathrm{~s}, 6 \mathrm{H}), 2.86-2.93(\mathrm{~m}, 6 \mathrm{H}) 6.78(\mathrm{~s}, 4 \mathrm{H}) 7.20-8.28(\mathrm{~m}$, $11 \mathrm{H}) .{ }^{13} \mathrm{C} \mathrm{NMR}\left(\mathrm{CDCl}_{3}, 100 \mathrm{MHz}, \mathrm{ppm}\right): \delta 152.4,152.3,151.4,144.3,144.2,144.1,140.5$, $140.1,140.0,138.5,138.0,134.7,129.5,127.7,127.6,126.1,125.6,124.2,124.1,123.6,121.8$, $56.4,56.3,56.2,28.9,28.7,23.1,20.8,20.8,8.1,8.0$. UV-vis (THF, $\left.1.0 \times 10^{-5} \mathrm{~mol} / \mathrm{L}\right) \lambda / \mathrm{nm}$ $\left(\varepsilon / \mathrm{dm}^{3} \cdot \mathrm{mol}^{-1} \cdot \mathrm{cm}^{-1}\right): 297$ (25 700), 308 (34 600), 358 (30 300). MALDI-TOF: $\mathrm{m} / z$ $758.9\left(\mathrm{M}^{+}\right)$, 729.7 ([M-29] $]^{+}$). Elemental Anal. Calcd. for $\mathrm{C}_{57} \mathrm{H}_{63} \mathrm{~B}: \mathrm{C}, 90.21 ; \mathrm{H}, 8.37$. Found: C, 90.40; H, 8.46. B2: a white powder, mp: 309-311 ${ }^{\circ} \mathrm{C} .{ }^{1} \mathrm{H}$ NMR $\left(\mathrm{CDCl}_{3}, 400 \mathrm{MHz}, \mathrm{ppm}\right) \delta 0.16-0.18$ (m, $18 \mathrm{H}), 2.07(\mathrm{~s}, 30 \mathrm{H}), 2.34(\mathrm{~s}, 12 \mathrm{H}), 2.93(\mathrm{~m}, 6 \mathrm{H}) 6.86(\mathrm{~s}, 8 \mathrm{H}) 7.30-8.28(\mathrm{~m}, 10 \mathrm{H}) .{ }^{13} \mathrm{C} \mathrm{NMR}$ $\left(\mathrm{CDCl}_{3}, 100 \mathrm{MHz}, \mathrm{ppm}\right): \delta 152.4,151.5,151.4,145.4,145.2,144.0,143.9,143.4,141.6,140.5$, $139.9,138.6,138.3,138.2,138.0,134.7,129.5,129.4,127.7,126.1,125.9,125.7,124.2$, 123.7, $123.6,121.8,56.4,56.3,56.2,29.2,28.9,28.7,23.1,20.8,8.1,8.0$. UV-vis (THF, $1.0 \times 10^{-5}$ $\mathrm{mol} / \mathrm{L}) \lambda / \mathrm{nm}\left(\varepsilon / \mathrm{dm}^{3} \cdot \mathrm{mol}^{-1} \cdot \mathrm{cm}^{-1}\right): 362(75800)$. MALDI-TOF: $m / z 1006.9\left(\mathrm{M}^{+}\right)$. Elemental Anal. Calcd. for $\mathrm{C}_{75} \mathrm{H}_{84} \mathrm{~B}_{2}$ : C, 89.45; H, 8.41. Found: C, 89.46; H, 8.45.

Synthesis of 2 -( $N, N$-diphenylamino)-5,5',10,10' $15,15^{\prime}$-hexaethyltruxene (N1) and 2,7-Di( $N, N$-diphenylamino)-5,5',10,10',15,15'-hexaethyltruxene (N2). A mixture of compound 9 (1.20 g, $1.35 \mathrm{mmol}), \mathrm{K}_{2} \mathrm{CO}_{3}(2.80 \mathrm{~g}, 20.3 \mathrm{mmol})$ powder, fresh $\mathrm{Cu}(0.30 \mathrm{~g}, 4.69$ mmol) powder, 18 - crown - 6 ether $(0.20 \mathrm{~g}, 0.83 \mathrm{mmol})$, diphenylamine $(1.20 \mathrm{~g}, 7.09 \mathrm{mmol})$ and 1,2-Dichlorobenzene $(20 \mathrm{~mL})$ were heated to reflux with stirring under nitrogen atmosphere. After reacting for further $8 \mathrm{~h}$, the mixture were cooled to room temperature and filtered off under suction. Then the filtrate was condensed, and purified by column chromatography on silica gel, eluting with dichloromethane-petrol ether (1:8) and compounds N1 $\left(0.16 \mathrm{~g}, 17 \%, R_{f}=0.56\right), \mathbf{N} 2\left(0.28 \mathrm{~g}, 25 \%, R_{f}=0.40\right)$ and $\mathbf{N} 3\left(0.71 \mathrm{~g}, 52 \%, R_{f}=0.33\right)$ were obtained. N1: a white powder, mp: $210-212{ }^{\circ} \mathrm{C} .{ }^{1} \mathrm{H}$ NMR $\left(\mathrm{CDCl}_{3}, 300 \mathrm{MHz}, \mathrm{ppm}\right) \delta 0.21(\mathrm{~s}$, 18H), 1.91-2.27 (m, 6H), 2.92-2.97(m, 6H), 6.80-7.42 (m, 18H) 8.15-8.35 (m, 3H). ${ }^{13} \mathrm{C}$ NMR $\left(\mathrm{CDCl}_{3}, 100 \mathrm{MHz}, \mathrm{ppm}\right): \delta 153.8,152.4,152.3,147.4,145.9,143.3,140.3,140.2,128.8,125.9$, $125.6,124.8,124.1,123.8,123.7,122.2,121.8,121.5,117.2,56.3,56.2,56.1,29.0,28.8,8.1$. UV-vis (THF, $\left.1.0 \times 10^{-5} \mathrm{~mol} / \mathrm{L}\right) \lambda / \mathrm{nm}\left(\varepsilon / \mathrm{dm}^{3} \cdot \mathrm{mol}^{-1} \cdot \mathrm{cm}^{-1}\right): 280$ (34 000), 294 (39 500), 306 (48 
000), 356 (28 600). MALDI-TOF: $m / z 675.8\left(\mathrm{M}^{+}\right), 646.9\left([\mathrm{M}-29]^{+}\right)$. Elemental Anal. Calcd. for $\mathrm{C}_{51} \mathrm{H}_{51} \mathrm{~N}$ : C, 90.35; H, 7.58; N, 2.07. Found: C, 90.26; H, 7.38; N, 2.16. N2: a white powder, mp: 282 - $284{ }^{\circ} \mathrm{C} .{ }^{1} \mathrm{H}$ NMR $\left(\mathrm{CDCl}_{3}, 300 \mathrm{MHz}, \mathrm{ppm}\right) \delta 0.22$ (s, 18H), 1.89-2.12 (m, 6H), 2.94(s, $6 \mathrm{H}), 6.92-7.41(\mathrm{~m}, 27 \mathrm{H})$ 8.08-8.28 (m, 3H). ${ }^{13} \mathrm{C} \mathrm{NMR}\left(\mathrm{CDCl}_{3}, 100 \mathrm{MHz}, \mathrm{ppm}\right): \delta 153.8,153.7$, $152.3,147.5,145.9,142.6,141.9,140.3,138.1,138.0,135.1,135.0,128.8,125.9$, 125.6, 124.9, $124.8,124.1,123.7,122.2,121.8,121.5,117.2,56.2,56.1,28.8,28.7,8.2,8.1$. UV-vis (THF, $\left.1.0 \times 10^{-5} \mathrm{~mol} / \mathrm{L}\right) \lambda / \mathrm{nm}\left(\varepsilon / \mathrm{dm}^{3} \cdot \mathrm{mol}^{-1} \cdot \mathrm{cm}^{-1}\right): 300$ (41 100), 360 (55 700). MALDI-TOF: $\mathrm{m} / z 844.5$ $\left(\mathrm{M}^{+}\right)$. Elemental Anal. Calcd. for $\mathrm{C}_{63} \mathrm{H}_{60} \mathrm{~N}_{2}$ : C, 89.53; H, 7.16; N, 3.31. Found: C, 89.69; H, $7.14 ; \mathrm{N}, 3.15$.

2,7-Dibromo-5,5' $\mathbf{1 0}^{\prime}, \mathbf{1 0}^{\prime}, \mathbf{1 5 , 1 5} \mathbf{1 5}^{\prime}$-hexaethyltruxene (2). To a stirred solution of 4 (2.00 g, 3.92 mmol) in 1,2-propanediol carbonate $(20 \mathrm{~mL}), \mathrm{N}$-bromosuccinimide (NBS) was added (1.42 g, $7.98 \mathrm{mmol}$ ) stepwise at $60{ }^{\circ} \mathrm{C}$ room temperature. After reacting for $2 \mathrm{~h}$ at the temperature, the mixture were poured into $100 \mathrm{~mL}$ water and extracted with dichloromethane. Then drying over magnesium sulfate, the crude product was afforded after removing solvent. After recrystalled with 1,2-propanediol carbonate and ethanol repeatly, compound 3 yielded $(2.40 \mathrm{~g}, 91 \%)$ as a white crystal, mp: $259-261{ }^{\circ} \mathrm{C} .{ }^{1} \mathrm{H}$ NMR $\left(\mathrm{CDCl}_{3}, 400 \mathrm{MHz}, \mathrm{ppm}\right) \delta$ 0.17-0.23 (m, 18H), 2.06-2.17 (m, 6H), 2.89-3.05 (m, 6H), 7.39-7.58 (m, 7H), 8.16-8.32 (m, 3H). UV-vis (THF, $\left.1.0 \times 10^{-5} \mathrm{~mol} / \mathrm{L}\right) \lambda / \mathrm{nm}\left(\varepsilon / \mathrm{dm}^{3} \cdot \mathrm{mol}^{-1} \cdot \mathrm{cm}^{-1}\right): 281$ (50 700), 300 (46 500), 312 (64 800). TOF-MS-EI: $m / z 668.0\left(M^{+}\right), 639.0\left([M-29]^{+}\right), 590.0\left([M-78]^{+}\right), 561\left([M-107]^{+}\right)$.

2,7-Dibromo-12-iodo-5,5',10,10',15,15'-hexaethyltruxene (3). A mixture of compound 1 $(2.10 \mathrm{~g}, 3.15 \mathrm{mmol})$ and $25 \mathrm{~mL}$ solvent $\left(\mathrm{CH}_{3} \mathrm{COOH}: \mathrm{H}_{2} \mathrm{SO}_{4}: \mathrm{H}_{2} \mathrm{O}: \mathrm{CCl}_{4}=100: 5: 20: 8\right)$ was heated to $40{ }^{\circ} \mathrm{C}$ with stirring. After adding $\mathrm{HIO}_{3}(0.19 \mathrm{~g}, 1.10 \mathrm{mmol})$ and $\mathrm{I}_{2}(0.53 \mathrm{~g}, 2.09 \mathrm{mmol})$ to the mixture, the mixture was heated to $80{ }^{\circ} \mathrm{C}$ and stirred for $4 \mathrm{~h}$ at the temperature. Afterward, the mixture was cooled to room temperature and filtered off under suction, washed with water. Then the residue refluxed in methanol for $2 \mathrm{~h}$ and followed by cooling to room temperature, filtered off under suction and 2 was afforded (2.20 g, $88 \%$ ) as a yellow powder, mp: 333 - 335 ${ }^{\circ} \mathrm{C} .{ }^{1} \mathrm{H}$ NMR $(\mathrm{CDCl} 3,400 \mathrm{MHz}, \mathrm{ppm}) \delta$ 0.19-0.22 (t, 18H, $\left.J=7.18\right), 2.06-2.14(\mathrm{~m}, 6 \mathrm{H})$, 2.88-2.93 (m, 6H), 7.50-8.17 (m, 9H). UV-vis (THF, $\left.1.0 \times 10^{-5} \mathrm{~mol} / \mathrm{L}\right) \lambda / \mathrm{nm}\left(\varepsilon / \mathrm{dm}^{3} \cdot \mathrm{mol}^{-1} \cdot \mathrm{cm}^{-1}\right)$ : 284 (62 800), 301 (58 500), 313 (101 900). TOF-MS-EI: $m / z 794.0\left(M^{+}\right), 765.0\left([M-29]^{+}\right)$. 
2,7-Dibromo-12-( $N, N$-diphenylamino)-5,5',10,10',15,15'-hexaethyltruxene (4). A mixture of compound 2 (1.80 g, $2.27 \mathrm{mmol}), \mathrm{K}_{2} \mathrm{CO}_{3}(2.00 \mathrm{~g}, 14.5 \mathrm{mmol})$ powder, fresh $\mathrm{Cu}(0.13 \mathrm{~g}, 2.03$ mmol) powder, 18 - crown - 6 ether $(0.20 \mathrm{~g}, 0.83 \mathrm{mmol})$, diphenylamine $(0.50 \mathrm{~g}, 2.96 \mathrm{mmol})$ and 1,2-Dichlorobenzene $(20 \mathrm{~mL})$ were heated to reflux with stirring under nitrogen atmosphere. After reacting for further $8 \mathrm{~h}$, the mixture were cooled to room temperature and filtered off under suction. Then the filtrate was condensed, and purified by column chromatography on silica gel, eluting with dichloromethane-petrol ether (1: 10) and compound 3 was obtained $(1.36 \mathrm{~g}, 72 \%)$ as a white powder, mp: $243-245{ }^{\circ} \mathrm{C} .{ }^{1} \mathrm{H}$ NMR $\left(\mathrm{CDCl}_{3}, 400 \mathrm{MHz}\right.$, ppm) $\delta 0.20-0.26(\mathrm{~m}, 18 \mathrm{H}), 1.94-2.10(\mathrm{~m}, 6 \mathrm{H}), 2.81-2.94(\mathrm{~m}, 6 \mathrm{H}), 6.99-7.56(\mathrm{~m}, 16 \mathrm{H}) 8.10-8.14$ (m, 3H). UV-vis (THF, $\left.1.0 \times 10^{-5} \mathrm{~mol} / \mathrm{L}\right) \lambda / \mathrm{nm}\left(\varepsilon / \mathrm{dm}^{3} \cdot \mathrm{mol}^{-1} \cdot \mathrm{cm}^{-1}\right): 285(45800), 301$ (44 100), 313 (52 700), 360 (29 000). MALDI-TOF: $m / z 835.5\left(M^{+}\right), 806.4\left([M-29]^{+}\right), 755.5\left([M-80]^{+}\right)$.

2-Bromo-5,5',10,10',15,15'-hexaethyltruxene (5). To a stirred solution of 4 (1.00 g, 1.96 mmol) in 1,2-propanediol carbonate $(15 \mathrm{~mL}), \mathrm{N}$-Bromosuccinimide (NBS) was added (0.35 g, $1.97 \mathrm{mmol}$ ) stepwise at $60{ }^{\circ} \mathrm{C}$ room temperature. After reacting for $2 \mathrm{~h}$ at the temperature, the mixture were cooled and filtrated to remove undissolved $\mathbf{1}$, and the filtrate was concentrated to the half. Afterward, the mixture were poured into $50 \mathrm{~mL}$ water and extracted with dichloromethane. Then drying over magnesium sulfate, the crude product was afforded after removing solvent. After recrystalled with ethanol, compound 5 yielded $(0.81 \mathrm{~g}, 70 \%)$ as a white crystal, mp: $232-234{ }^{\circ} \mathrm{C} .{ }^{1} \mathrm{H}$ NMR $\left(\mathrm{CDCl}_{3}, 300 \mathrm{MHz}, \mathrm{ppm}\right) \delta 0.19$ (s, 18H), 2.08 (s, 6H), 2.86-3.03 (m, 6H), 7.25-8.30 (m, 11H). UV-vis (THF, $\left.1.0 \times 10^{-5} \mathrm{~mol} / \mathrm{L}\right) \lambda / \mathrm{nm}\left(\varepsilon / \mathrm{dm}^{3} \cdot \mathrm{mol}^{-1} \cdot \mathrm{cm}^{-1}\right)$ : 278 (42 200), 294 (41 600), 306 (70 800). TOF-MS-EI: $m / z 588.0\left(\mathrm{M}^{+}\right), 559.0\left([M-29]^{+}\right), 510.0$ $\left([M-78]^{+}\right)$.

2-Bromo-7,12-diiodo-5,5',10,10',15,15'-hexaethyltruxene (6). A mixture of compound 5 (0.70 g, $1.19 \mathrm{mmol})$ and $15 \mathrm{~mL}$ solvent $\left(\mathrm{CH}_{3} \mathrm{COOH}: \mathrm{H}_{2} \mathrm{SO}_{4}: \mathrm{H}_{2} \mathrm{O}: \mathrm{CCl}_{4}=100: 5: 20: 8\right)$ was heated to $40{ }^{\circ} \mathrm{C}$ with stirring. After adding $\mathrm{HIO}_{3}(0.11 \mathrm{~g}, 0.60 \mathrm{mmol})$ and $\mathrm{I}_{2}(0.30 \mathrm{~g}, 1.19 \mathrm{mmol})$ to the mixture, the mixture was heated to $80{ }^{\circ} \mathrm{C}$ and stirred for $4 \mathrm{~h}$ at the temperature. Afterward, the mixture was cooled to room temperature and filtered off under suction, washed with water. Then the residue refluxed in methanol for $2 \mathrm{~h}$ and followed by cooling to room temperature, filtered off under suction and 6 was afforded $(0.86 \mathrm{~g}, 86 \%)$ as a yellow powder, mp: 330 - 332 ${ }^{\circ} \mathrm{C} .{ }^{1} \mathrm{H}$ NMR $\left(\mathrm{CDCl}_{3}, 400 \mathrm{MHz}, \mathrm{ppm}\right) \delta 0.18(\mathrm{~s}, 18 \mathrm{H}), 2.04-2.10(\mathrm{~m}, 6 \mathrm{H}), 2.85-3.03(\mathrm{~m}, 6 \mathrm{H})$, 
7.38-8.30 (m, 9H). UV-vis (THF, $\left.1.0 \times 10^{-5} \mathrm{~mol} / \mathrm{L}\right) \lambda / \mathrm{nm}\left(\varepsilon / \mathrm{dm}^{3} \cdot \mathrm{mol}^{-1} \cdot \mathrm{cm}^{-1}\right): 286(52900), 303$ (58 300), 316 (94 400). TOF-MS-EI: $m / z 840.0\left(M^{+}\right), 811.0\left([M-29]^{+}\right)$.

2-Bromo-7,12-di( $N, N$-diphenylamino)-5,5',10,10',15,15'-hexaethyltruxene (7). A mixture of compound 6 (0.75 g, $0.89 \mathrm{mmol}), \mathrm{K}_{2} \mathrm{CO}_{3}(1.00 \mathrm{~g}, 7.25 \mathrm{mmol})$ powder, fresh $\mathrm{Cu}(0.07 \mathrm{~g}, 1.09$ mmol) powder, 18 - crown - 6 ether $(0.15 \mathrm{~g}, 0.62 \mathrm{mmol})$, diphenylamine $(0.31 \mathrm{~g}, 1.83 \mathrm{mmol})$ and 1,2-Dichlorobenzene $(15 \mathrm{~mL})$ were heated to reflux with stirring under nitrogen atmosphere. After reacting for further $8 \mathrm{~h}$, the mixture were cooled to room temperature and filtered off under suction. Then the filtrate was condensed, and purified by column chromatography on silica gel, eluting with dichloromethane-petrol ether (1:10) and compound 7 was obtained $(0.56 \mathrm{~g}, 69 \%)$ as a white powder, mp: $232-235{ }^{\circ} \mathrm{C} .{ }^{1} \mathrm{H}$ NMR $\left(\mathrm{CDCl}_{3}, 400 \mathrm{MHz}\right.$, ppm) $\delta$ 0.20-0.28 (m, 18H), 1.90-2.08 (m, 6H), 2.81-2.94(m, 6H), 7.00-7.54 (m, 26H) 8.08-8.13 (m, 3H). UV-vis (THF, $\left.1.0 \times 10^{-5} \mathrm{~mol} / \mathrm{L}\right) \lambda / \mathrm{nm}\left(\varepsilon / \mathrm{dm}^{3} \cdot \mathrm{mol}^{-1} \cdot \mathrm{cm}^{-1}\right): 301$ (52 000), 362 (51 800$)$. MALDI-TOF: $m / z 924.8\left(\mathrm{M}^{+}\right), 895.6\left([M-29]^{+}\right), 844.7\left([M-80]^{+}\right), 844.7\left([M-109]^{+}\right)$. 


\section{Absorption and emission graphics}
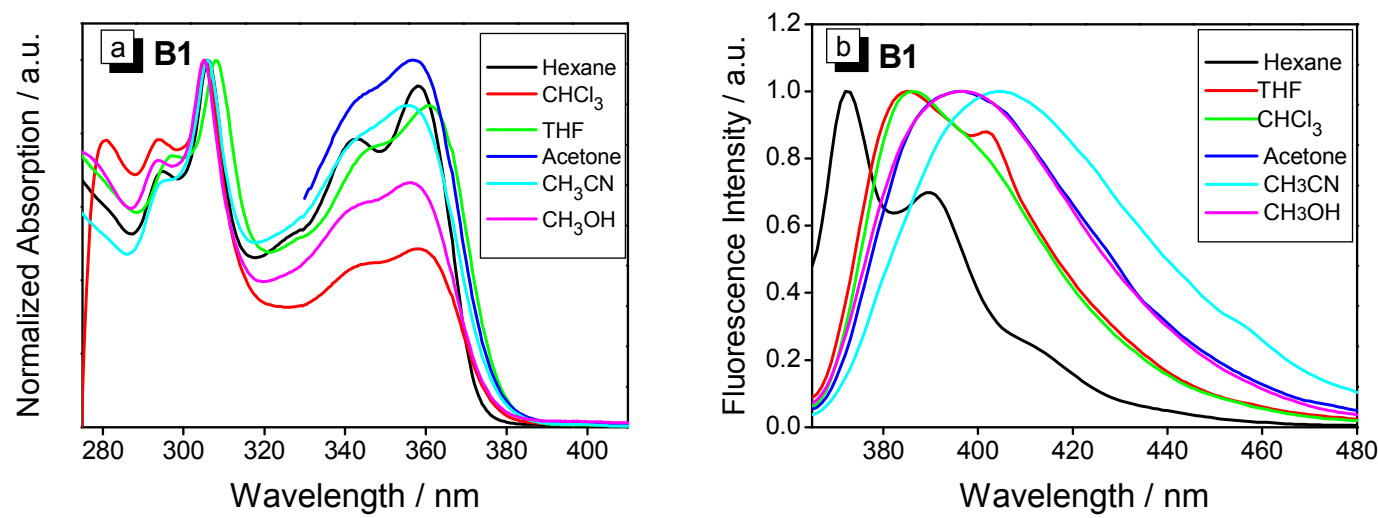

Figure S1 Normalized absorption (a) and emission (b) spectra of B1 $\left(c=1.0 \times 10^{-5} \mathrm{~mol} / \mathrm{L}, \lambda_{\mathrm{ex}}\right.$ $=360 \mathrm{~nm}$ ) in different solvents.
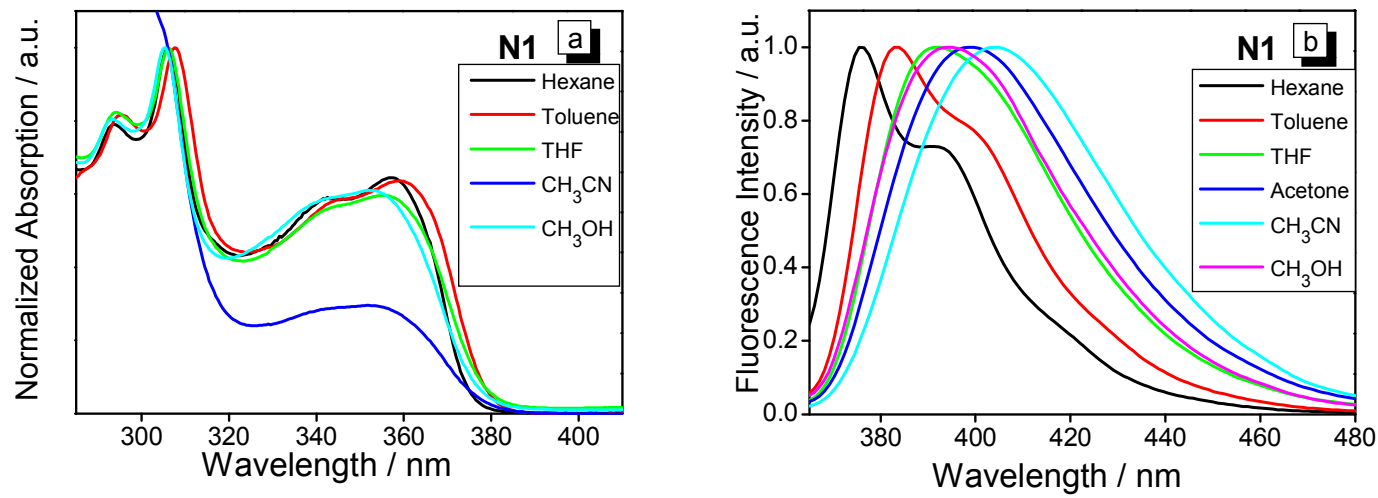

Figure S2 Normalized absorption (a) and emission (b) spectra of N1 ( $c=1.0 \times 10^{-5} \mathrm{~mol} / \mathrm{L}, \lambda_{\mathrm{ex}}$ $=360 \mathrm{~nm}$ ) in different solvents.
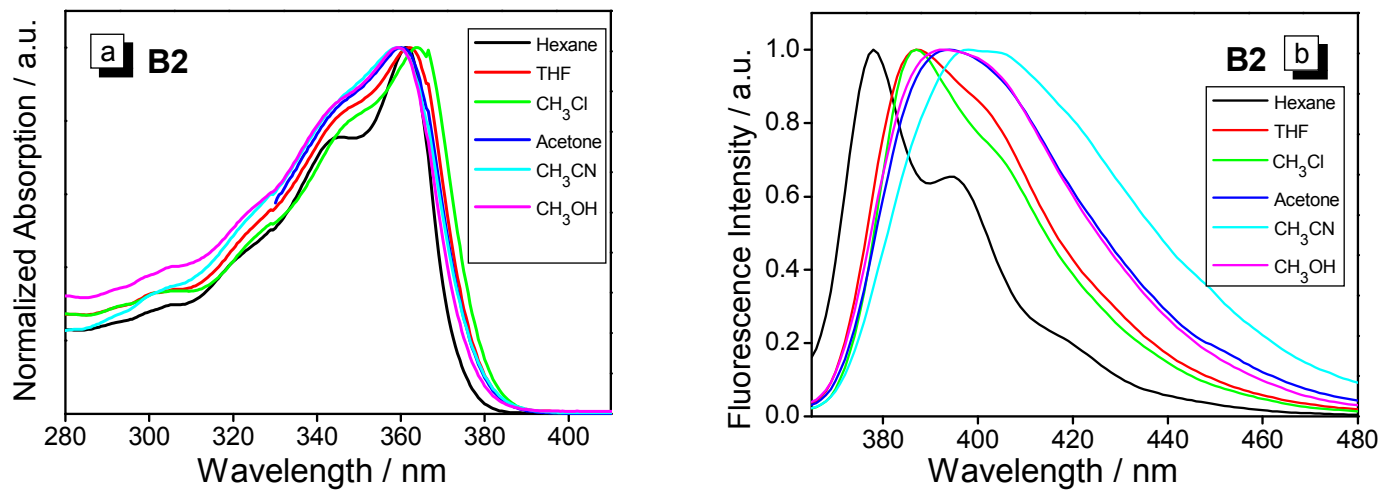

Figure S3 Normalized absorption (a) and emission (b) spectra of $\mathbf{B 2}\left(c=1.0 \times 10^{-5} \mathrm{~mol} / \mathrm{L}, \lambda_{\mathrm{ex}}\right.$ $=360 \mathrm{~nm}$ ) in different solvents. 

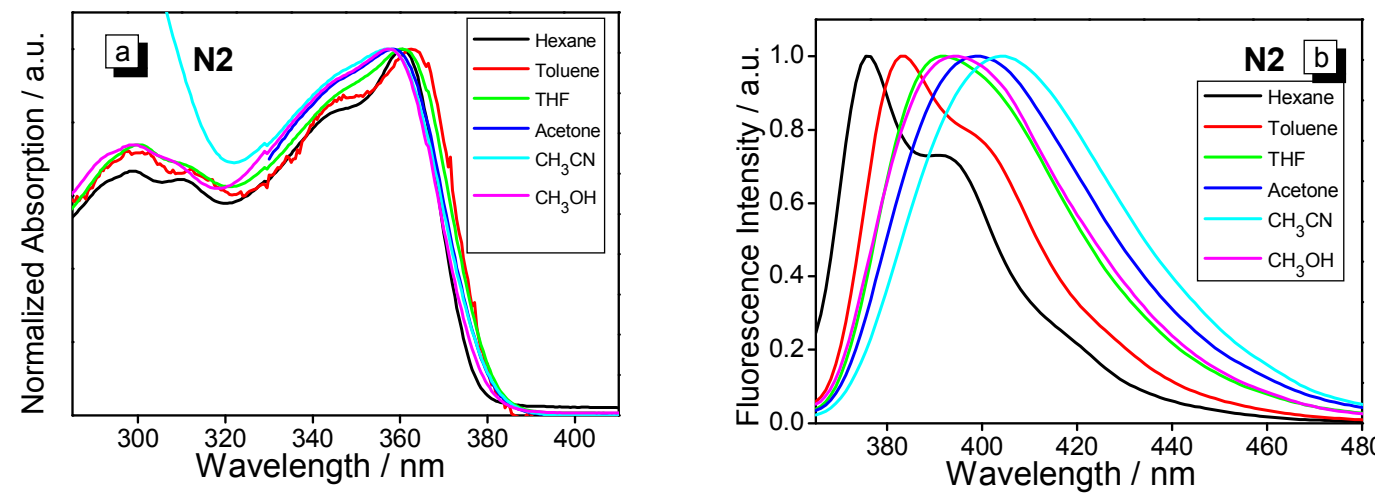

Figure S4 Normalized absorption (a) and emission (b) spectra of N2 $\left(c=1.0 \times 10^{-5} \mathrm{~mol} / \mathrm{L}, \lambda_{\mathrm{ex}}\right.$ $=360 \mathrm{~nm}$ ) in different solvents.
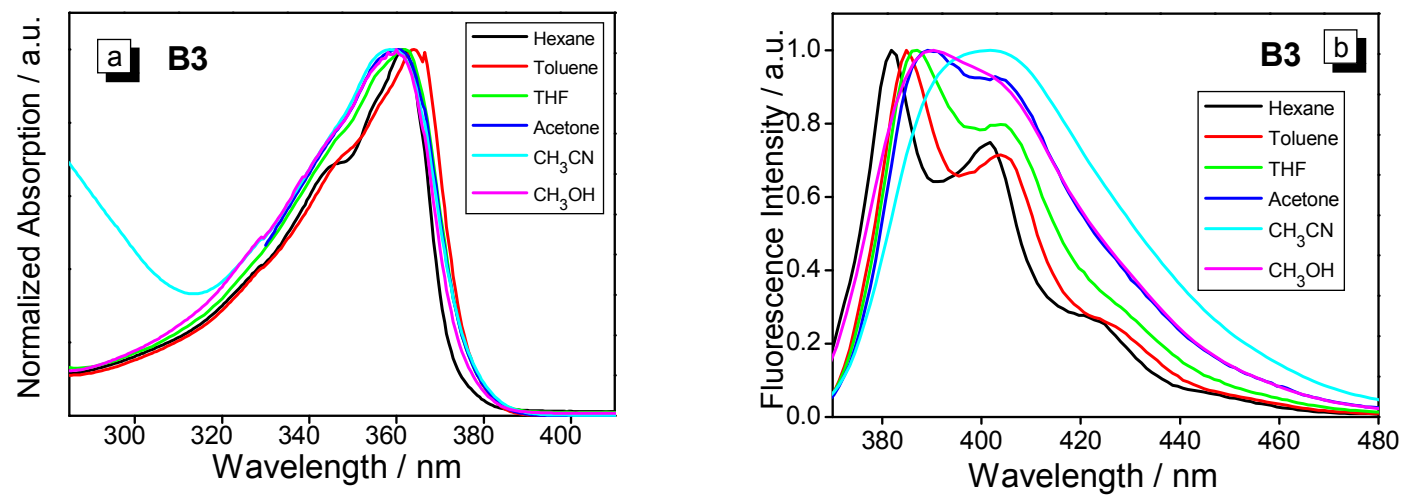

Figure S5 Normalized absorption (a) and emission (b) spectra of B3 $\left(c=1.0 \times 10^{-5} \mathrm{~mol} / \mathrm{L}, \lambda_{\mathrm{ex}}\right.$ $=360 \mathrm{~nm}$ ) in different solvents.
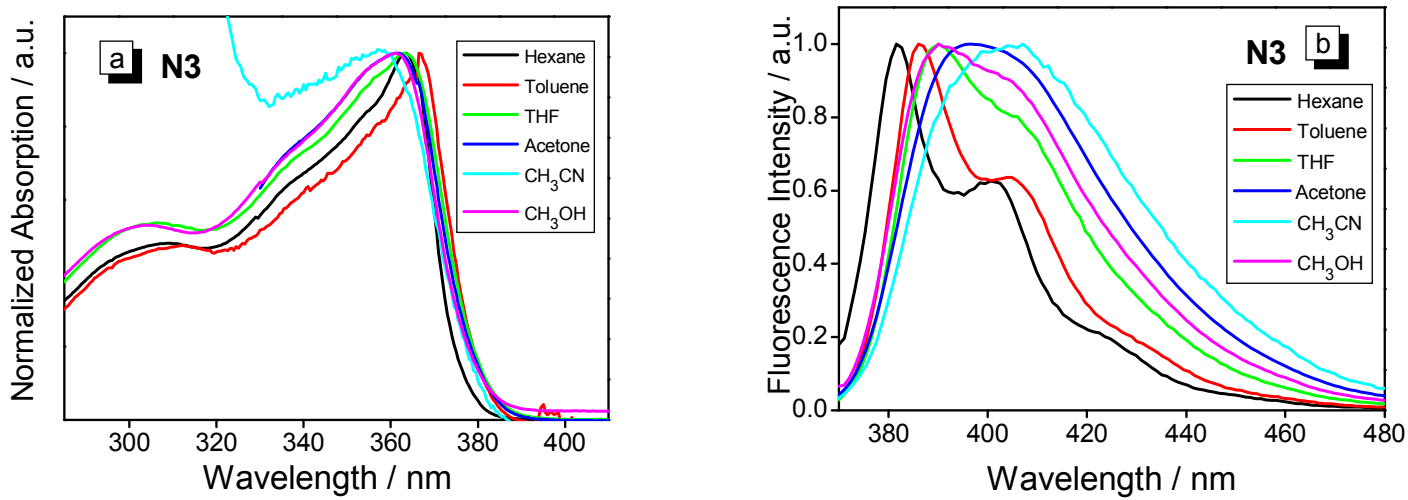

Figure S6 Normalized absorption (a) and emission (b) spectra of $\mathbf{N 3}\left(c=1.0 \times 10^{-5} \mathrm{~mol} / \mathrm{L}, \lambda_{\mathrm{ex}}\right.$ $=360 \mathrm{~nm}$ ) in different solvents. 

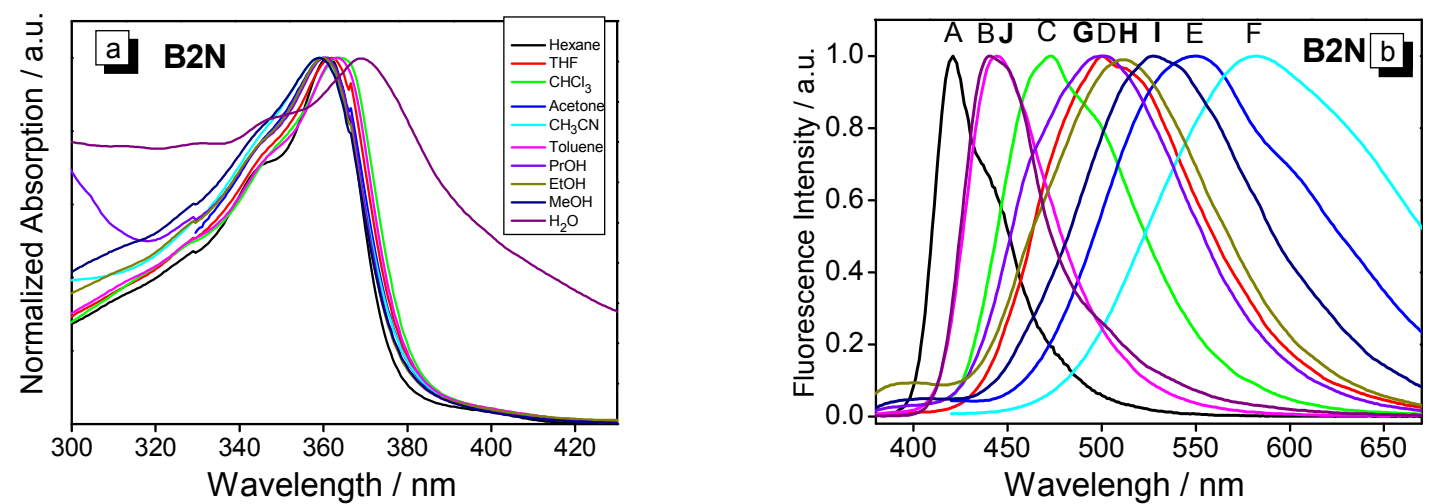

Figure S7 Normalized absorption (a) and emission (b) spectra of B2N $\left(c=1.0 \times 10^{-5} \mathrm{~mol} / \mathrm{L}\right.$, $\left.\lambda_{\mathrm{ex}}=360 \mathrm{~nm}\right)$ in different solvents.
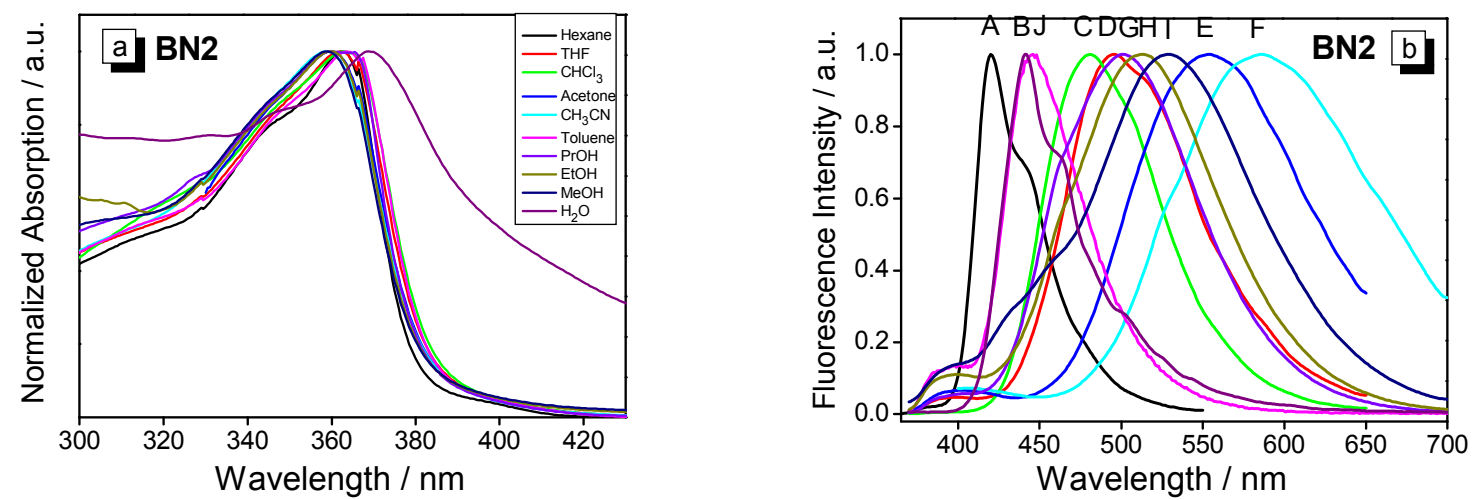

Figure S8 Normalized absorption (a) and emission (b) spectra of BN2 $\left(c=1.0 \times 10^{-5} \mathrm{~mol} / \mathrm{L}\right.$, $\left.\lambda_{\mathrm{ex}}=360 \mathrm{~nm}\right)$ in different solvents.

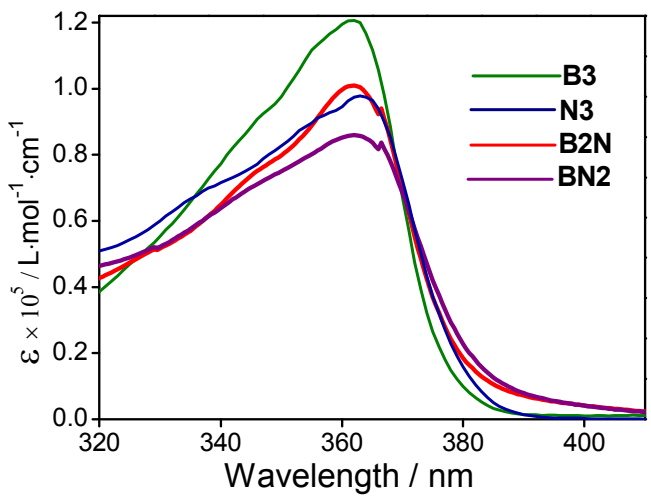

Figure S9 Absorption of B3, N3, BN2 and B2N in THF with $c=1.0 \times 10^{-5} \mathrm{~mol} / \mathrm{L}$. 


\section{Absorption and emission data}

Table 3. Absorption and emission data of BN2, B2N, N1, N2, N3, B1, B2, B3 and 6DMN-GlyOMe ${ }^{6}$ in various solvents.

\begin{tabular}{|c|c|c|c|c|c|c|c|c|c|c|c|c|c|c|c|c|c|c|c|c|}
\hline & \multicolumn{2}{|c|}{ hexane } & \multicolumn{2}{|c|}{ toluene } & \multicolumn{2}{|c|}{$\mathrm{CHCl}_{3}$} & \multicolumn{2}{|c|}{ THF } & \multicolumn{2}{|c|}{ acetone } & \multicolumn{2}{|c|}{$\mathrm{CH}_{3} \mathrm{CN}$} & \multicolumn{2}{|c|}{$n-\mathrm{PrOH}$} & \multicolumn{2}{|c|}{$\mathrm{EtOH}$} & \multicolumn{2}{|c|}{$\mathrm{MeOH}$} & \multicolumn{2}{|c|}{$\mathrm{H}_{2} \mathrm{O}$} \\
\hline & $\lambda_{\mathrm{abs}}$ & $\lambda_{\mathrm{em}}$ & $\lambda_{\mathrm{abs}}$ & $\lambda_{\mathrm{em}}$ & $\lambda_{\mathrm{abs}}$ & $\lambda_{\mathrm{em}}$ & $\lambda_{\mathrm{abs}}$ & $\lambda_{\mathrm{em}}$ & $\lambda_{\mathrm{abs}}$ & $\lambda_{\mathrm{em}}$ & $\lambda_{\mathrm{abs}}$ & $\lambda_{\mathrm{em}}$ & $\lambda_{\mathrm{abs}}$ & $\lambda_{\mathrm{em}}$ & $\lambda_{\mathrm{abs}}$ & $\lambda_{\mathrm{em}}$ & $\lambda_{\mathrm{abs}}$ & $\lambda_{\mathrm{em}}$ & $\lambda_{\mathrm{abs}}$ & $\lambda_{\mathrm{em}}$ \\
\hline BN2 & 362 & 420 & 363 & 446 & 363 & 481 & 362 & 496 & 360 & 554 & 359 & 586 & 360 & 501 & 360 & 513 & 359 & 529 & 368 & 442 \\
\hline B2N & 361 & 421 & 363 & 444 & 364 & 473 & 362 & 500 & 360 & 550 & 359 & 582 & 360 & 501 & 360 & 512 & 359 & 528 & 369 & 441 \\
\hline 6DMN-GlyOM ${ }^{a}$ & - & - & 373 & 491 & 380 & 509 & 373 & 510 & 375 & 532 & 380 & 549 & - & - & 379 & 584 & 382 & 589 & 388 & 592 \\
\hline N1 & 356 & 378 & 360 & 383 & - & - & 356 & 392 & 354 & 399 & 353 & 404 & - & - & - & - & 352 & 394 & - & - \\
\hline $\mathbf{N} 2$ & 360 & 379 & 362 & 384 & - & - & 360 & 389 & 358 & 396 & 357 & 402 & - & - & - & - & 357 & 391 & - & - \\
\hline $\mathbf{N 3}$ & 364 & 382 & 366 & 386 & - & - & 363 & 390 & 361 & 395 & 359 & 404 & - & - & - & - & 360 & 391 & - & - \\
\hline B1 & 358 & 372 & - & - & 361 & 387 & 358 & 385 & 357 & 395 & 356 & 404 & - & - & - & - & 357 & 398 & - & - \\
\hline B2 & 362 & 378 & - & - & 362 & 387 & 364 & 387 & 360 & 394 & 359 & 398 & - & - & - & - & 359 & 394 & - & - \\
\hline B3 & 362 & 382 & 364 & 385 & - & - & 362 & 387 & 360 & 396 & 359 & 403 & - & - & - & - & 359 & 397 & - & - \\
\hline$\Delta f$ & \multicolumn{2}{|c|}{0.001} & \multicolumn{2}{|c|}{0.013} & \multicolumn{2}{|c|}{0.150} & \multicolumn{2}{|c|}{0.210} & \multicolumn{2}{|c|}{0.284} & \multicolumn{2}{|c|}{0.306} & \multicolumn{2}{|c|}{0.250} & \multicolumn{2}{|c|}{0.290} & \multicolumn{2}{|c|}{0.307} & \multicolumn{2}{|c|}{0.320} \\
\hline
\end{tabular}

\section{Titration experiments of $\mathrm{B} 2 \mathrm{~N}$ and $\mathrm{BN} 2$ with $\mathrm{Cl}^{-}$and $\mathrm{Br}^{-}$}
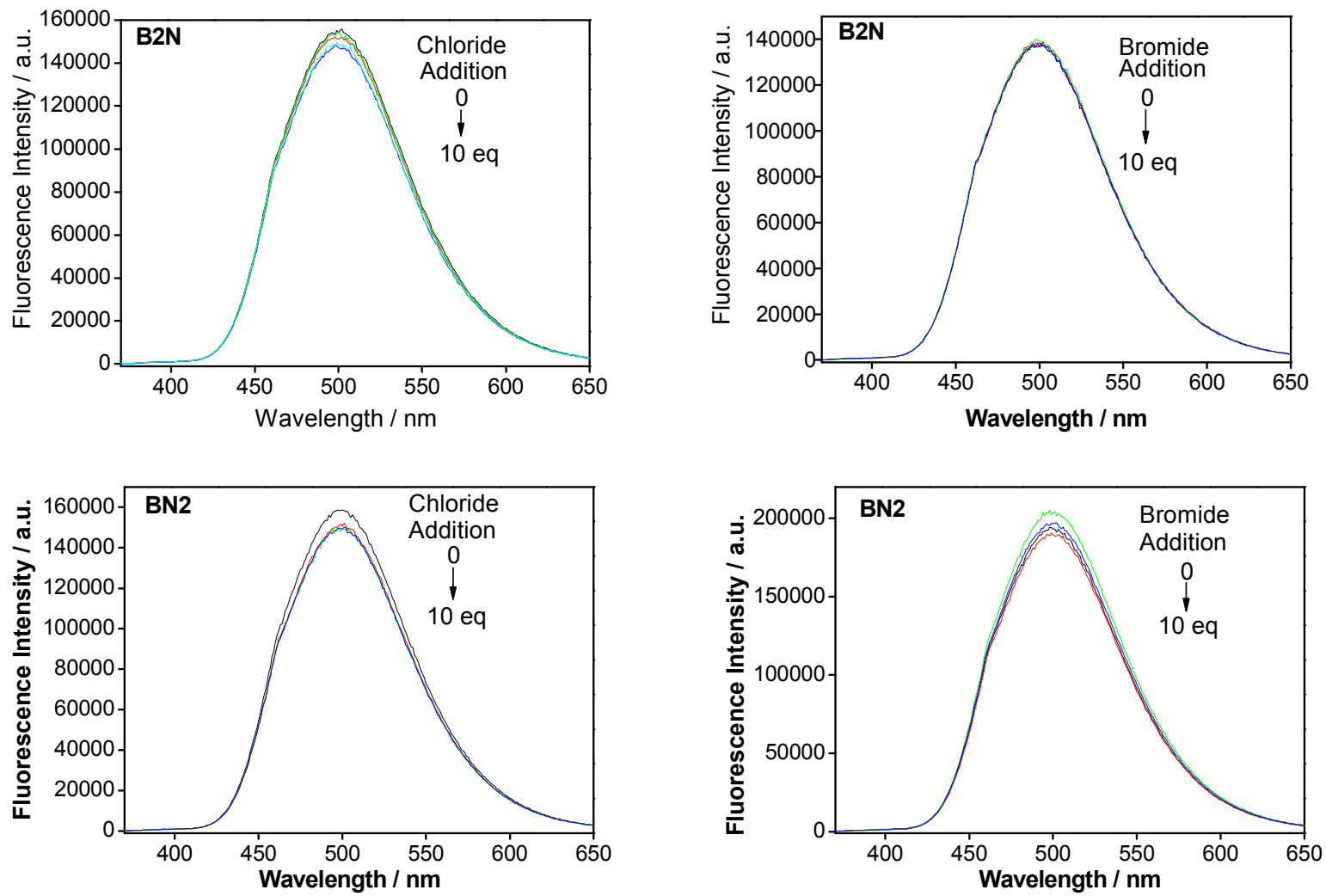

Figure S10 Fluorescence spectral changes of B2N and BN2 in THF with $c=1.0 \times 10^{-5} \mathrm{~mol} / \mathrm{L}$ upon the addition of ten equiv chloride and bromide anions with THF solutions of their $\mathrm{Bu}_{4} \mathrm{~N}^{+}$ salts. 


\section{Calculation of the association constant ${ }^{20}$}

\section{(5a) BN2}

A THF solution of BN2 $\left(2 \mathrm{~mL}, 1 \times 10^{-5} \mathrm{M}\right)$ was placed in the cell and was titrated with incremental amounts of fluoride anions by addition of a solution of $n \mathrm{Bu}_{4} \mathrm{NF}$ in THF $\left(4 \times 10^{-4}\right.$ M). The absorption at $362 \mathrm{~nm}$ was monitored.

$$
\mathrm{BN} 2 \quad+\quad \mathrm{F}^{-} \quad \Leftrightarrow \quad \text { FBN2 }
$$

$\mathrm{I}=\mathrm{K}_{\mathrm{s}}[\mathrm{BN} 2]+\mathrm{K}_{\mathrm{p}}[\mathrm{FBN} 2]$

$\mathrm{I}_{0}=\mathrm{K}_{\mathrm{s}}[\mathrm{BN} 2]_{0}$

$[\mathrm{BN} 2]_{0}=[\mathrm{BN} 2]+[\mathrm{FBN} 2]$

$\mathrm{K}=[\mathrm{FBN} 2] /[\mathrm{BN} 2][\mathrm{F}]$

$\mathrm{I} / \mathrm{I}_{0}=\left(1+\left(\mathrm{K}_{\mathrm{p}} / \mathrm{K}_{\mathrm{s}}\right) \mathrm{K}[\mathrm{F}]\right) /(1+\mathrm{K}[\mathrm{F}]) \quad \longrightarrow\left(1-\mathrm{I} / \mathrm{I}_{0}\right) /[\mathrm{F}]=\mathrm{K}\left(\mathrm{I} / \mathrm{I}_{0}\right)-\mathrm{K}\left(\mathrm{K}_{\mathrm{p}} / \mathrm{K}_{\mathrm{s}}\right)$

From the slop of $\left(1-\mathrm{I} / \mathrm{I}_{0}\right) /[\mathrm{F}]$ vs $\mathrm{I} / \mathrm{I}_{0}$, the binding constant of $\mathbf{B N 2} \mathrm{K}=2.5 \times 10^{5} \mathrm{M}^{-1}$ was obtained.

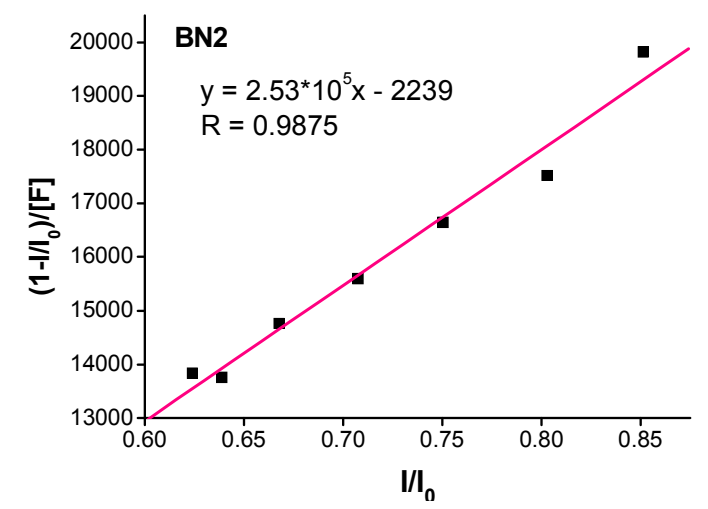

Figure S11 The fitting result of absorption titration data of BN2 with fluoride ion.

\section{(5b) $\mathrm{B} 2 \mathrm{~N}$}

A THF solution of B2N $\left(2 \mathrm{~mL}, 1 \times 10^{-5} \mathrm{M}\right)$ was placed in the cell and was titrated with incremental amounts of fluoride anions by addition of a solution of $n \mathrm{Bu}_{4} \mathrm{NF}$ in THF $\left(4 \times 10^{-4} \mathrm{M}\right)$. The absorption at $362 \mathrm{~nm}$ was monitored.

$\begin{array}{cccc}\mathrm{B} 2 \mathrm{~N} & + & 2 \mathrm{~F}^{-} & \Leftrightarrow\end{array} \quad \mathrm{F} 2 \mathrm{~B} 2 \mathrm{~N}$




$$
\mathrm{I}_{0}=\mathrm{K}_{\mathrm{s}}[\mathrm{B} 2 \mathrm{~N}]_{0}
$$$$
[\mathrm{B} 2 \mathrm{~N}]_{0}=[\mathrm{B} 2 \mathrm{~N}]+[\mathrm{F} 2 \mathrm{~B} 2 \mathrm{~N}]
$$$$
\mathrm{K}=[\mathrm{F} 2 \mathrm{~B} 2 \mathrm{~N}] /[\mathrm{B} 2 \mathrm{~N}][\mathrm{F}]^{2}
$$$$
\mathrm{I} / \mathrm{I}_{0}=\left(1+\left(\mathrm{K}_{\mathrm{p}} / \mathrm{K}_{\mathrm{s}}\right) \mathrm{K}[\mathrm{F}]^{2}\right) /\left(1+\mathrm{K}[\mathrm{F}]^{2}\right) \longrightarrow \quad\left(1-\mathrm{I} / \mathrm{I}_{0}\right) /[\mathrm{F}]^{2}=\mathrm{K}\left(\mathrm{I} / \mathrm{I}_{0}\right)-\mathrm{K}\left(\mathrm{K}_{\mathrm{p}} / \mathrm{K}_{\mathrm{s}}\right)
$$$$
\mathrm{K}_{1}=3.1 \times 10^{5} \mathrm{M}^{-1} \text { and } \mathrm{K}_{2} \text { (total) }=6.9 \times 10^{9} \mathrm{M}^{-2}
$$
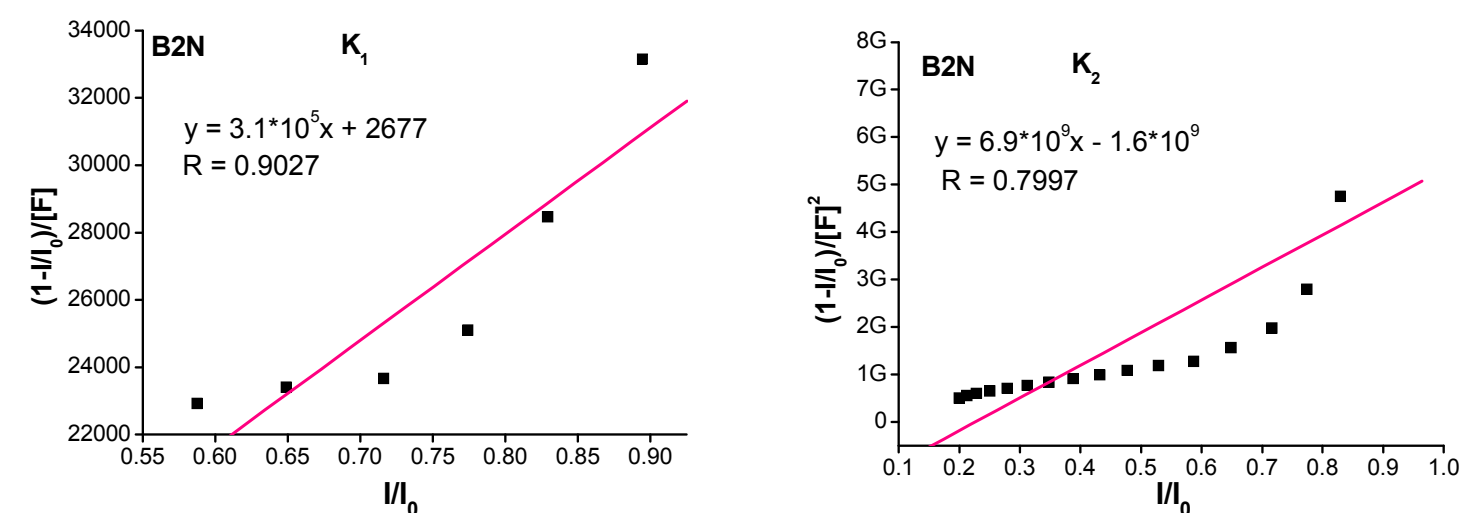

Figure S12 The fitting result of absorption titration data of B2N with fluoride ion.

\section{(5c) B3}

A THF solution of $\mathbf{B 3}\left(2 \mathrm{~mL}, 1 \times 10^{-5} \mathrm{M}\right)$ was placed in the cell and was titrated with incremental amounts of fluoride anions by addition of a solution of $n \mathrm{Bu}_{4} \mathrm{NF}$ in THF $\left(4 \times 10^{-4} \mathrm{M}\right)$. The absorption at $360 \mathrm{~nm}$ was monitored.
B3
$+\quad 3 \mathrm{~F}^{-}$
$\Leftrightarrow$
F3B3

$\mathrm{I}=\mathrm{K}_{\mathrm{s}}[\mathrm{B} 3]+\mathrm{K}_{\mathrm{p}}[\mathrm{F} 3 \mathrm{~B} 3]$

$\mathrm{I}_{0}=\mathrm{K}_{\mathrm{s}}[\mathrm{B} 3]_{0}$

$[\mathrm{B} 3]_{0}=[\mathrm{B} 3]+[\mathrm{F} 3 \mathrm{~B} 3]$

$\mathrm{K}=[\mathrm{F} 3 \mathrm{~B} 3] /[\mathrm{B} 3][\mathrm{F}]^{3}$

$\mathrm{I} / \mathrm{I}_{0}=\left(1+\left(\mathrm{K}_{\mathrm{p}} / \mathrm{K}_{\mathrm{s}}\right) \mathrm{K}[\mathrm{F}]^{3}\right) /\left(1+\mathrm{K}[\mathrm{F}]^{3}\right) \longrightarrow \quad\left(1-\mathrm{I} / \mathrm{I}_{0}\right) /[\mathrm{F}]^{3}=\mathrm{K}\left(\mathrm{I} / \mathrm{I}_{0}\right)-\mathrm{K}\left(\mathrm{K}_{\mathrm{p}} / \mathrm{K}_{\mathrm{s}}\right)$

$\mathrm{K}_{1}=3.9 \times 10^{5} \mathrm{M}^{-1}, \mathrm{~K}_{2 \text { (total) }}=9.7 \times 10^{9} \mathrm{M}^{-2}$ and $\mathrm{K}_{3 \text { (total) }}=1.6 \times 10^{14} \mathrm{M}^{-3}$ 

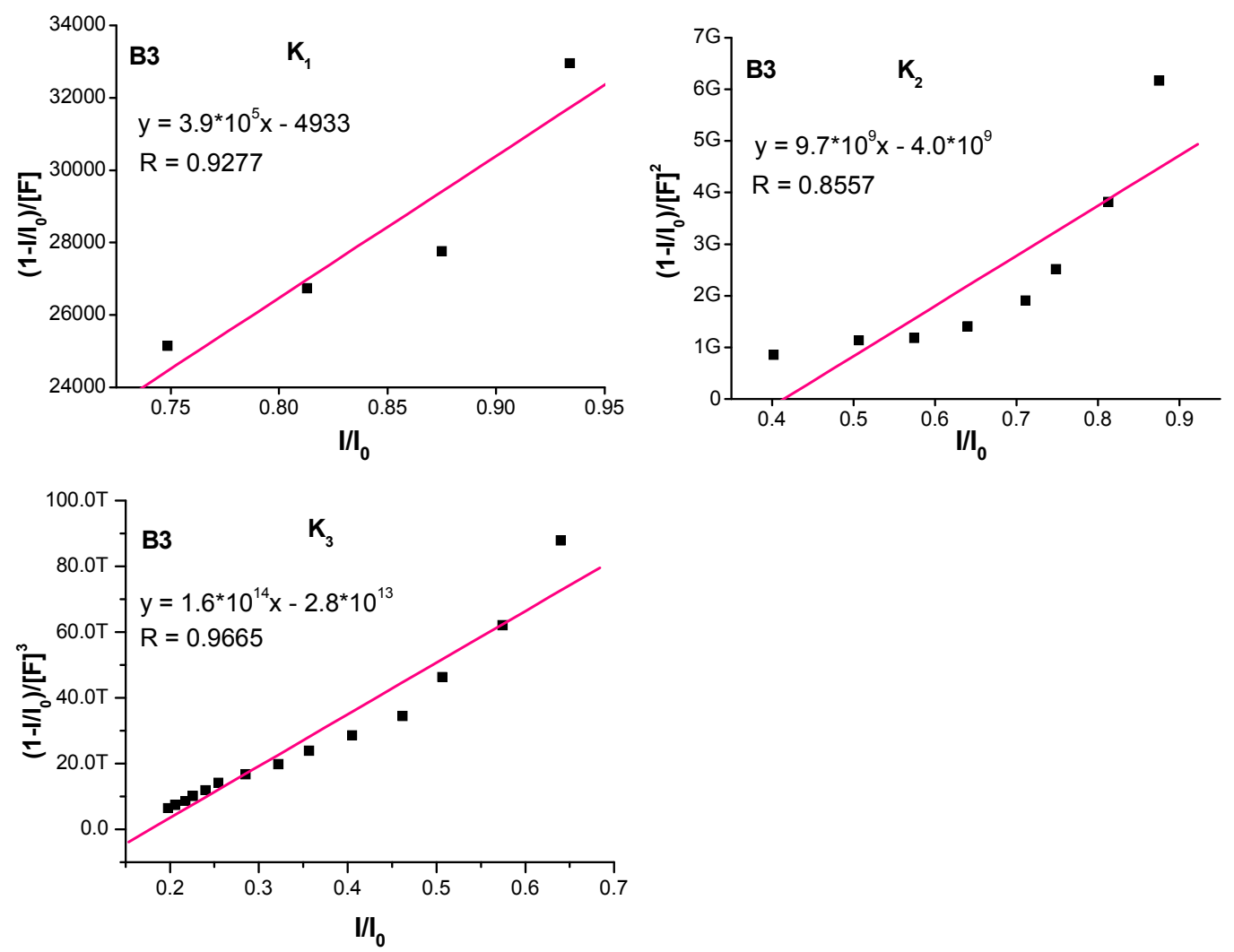

Figure S13 The fitting result of absorption titration data of B3 with fluoride ion.

(5d) B2
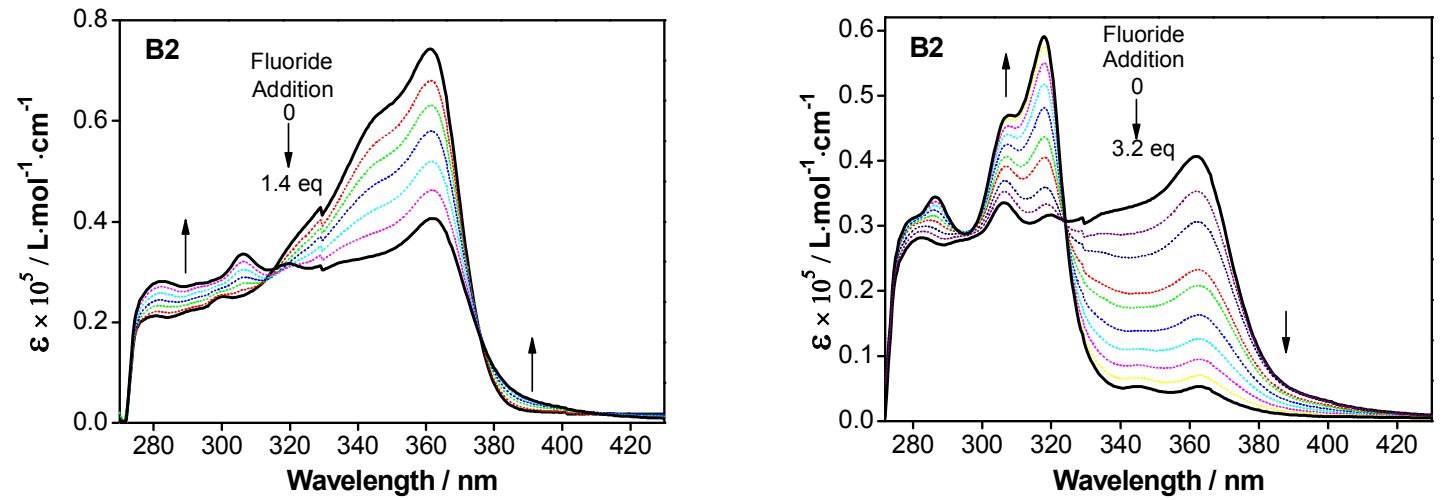

Figure S14 Absorption spectra for the titration of B2 $\left(c=1.0 \times 10^{-5} \mathrm{~mol} / \mathrm{L}\right.$ in THF) with $n$-Bu ${ }_{4} \mathrm{NF}$ solution of THF.

A THF solution of $\mathbf{B 2}\left(2 \mathrm{~mL}, 1 \times 10^{-5} \mathrm{M}\right)$ was placed in the cell and was titrated with incremental amounts of fluoride anions by addition of a solution of $n \mathrm{Bu}_{4} \mathrm{NF}$ in THF $\left(4 \times 10^{-4} \mathrm{M}\right)$. The absorption at $362 \mathrm{~nm}$ was monitored.

$\mathrm{K}_{1}=2.9 \times 10^{5} \mathrm{M}^{-1}$ and $\mathrm{K}_{2 \text { (total) }}=8.0 \times 10^{9} \mathrm{M}^{-2}$ 

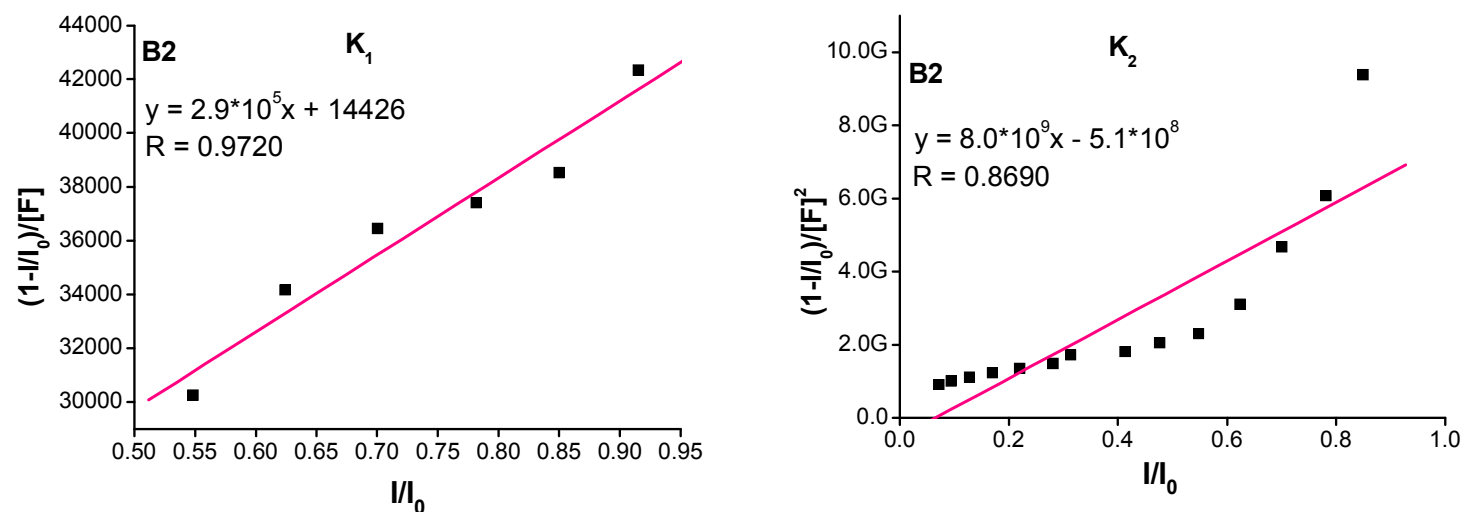

Figure S15 The fitting result of absorption titration data of B2 with fluoride ion.

\section{(5e) B1}

A THF solution of $\mathbf{B} 1\left(2 \mathrm{~mL}, 1 \times 10^{-5} \mathrm{M}\right)$ was placed in the cell and was titrated with incremental amounts of fluoride anions by addition of a solution of $n \mathrm{Bu}_{4} \mathrm{NF}$ in THF $\left(4 \times 10^{-4} \mathrm{M}\right)$. The absorption at $362 \mathrm{~nm}$ was monitored.

$\mathrm{K}_{1}=3.1 \times 10^{5} \mathrm{M}^{-1}$
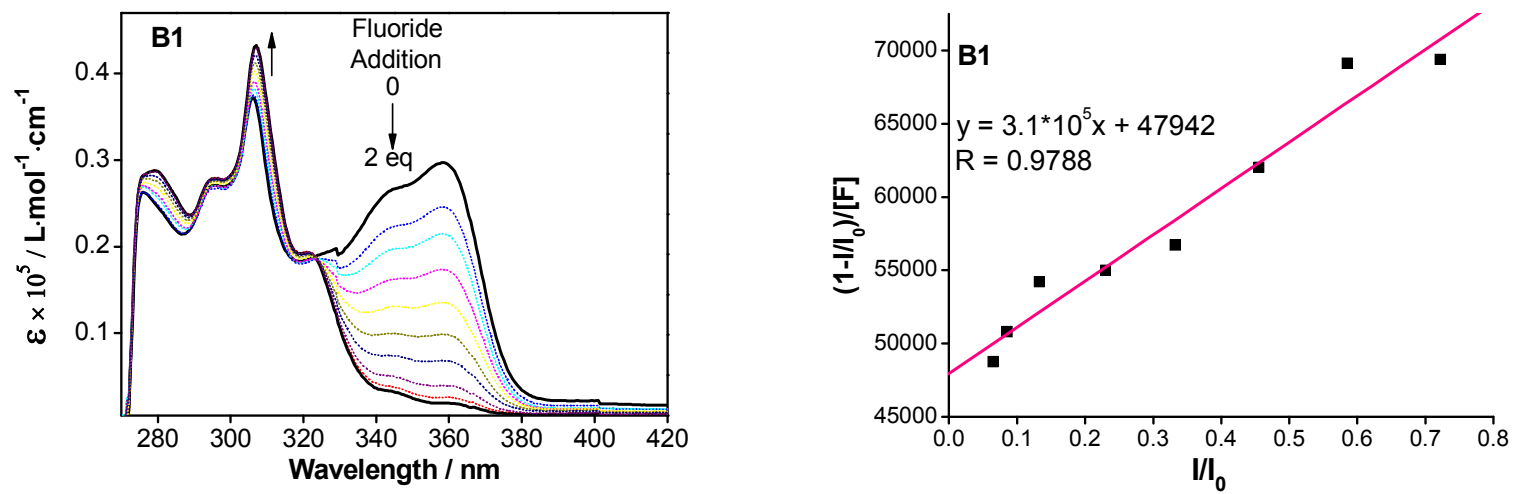

Figure S16 Absorption spectra for the titration of B1 $\left(c=1.0 \times 10^{-5} \mathrm{~mol} / \mathrm{L}\right.$ in THF) with $n$ - $\mathrm{Bu}_{4} \mathrm{NF}$ solution of THF (left) and the fitting result of absorption titration data of $\mathbf{B 1}$ with fluoride ion (right). 


\section{Computational work and the results}

By using the Gaussian 03 program, ${ }^{26}$ the Time-Dependent Density Functional Theory (TDDFT) and the B3LYP scheme in conjunction with the 6-31G(d) basis set has been used to carry out the study of compounds B3, N3, B2N and BN2.

Because the molecular size of $\mathbf{B 2 N}$ and $\mathbf{B N 2}$ are too large and to keep the computation feasible, all ethyl groups in the 5, 10, 15 positions of truxene and the methyl groups in the mesityl groups have been replaced by H-atom and the resultant compounds are named $\mathbf{B 3}^{\prime}, \mathbf{N 3}^{\prime}$, $\mathbf{B 2 N}^{\prime}$ and $\mathbf{B N 2}{ }^{\prime}$ respectively.

The molecular geometry for electronic structure calculation was obtained by DFT-B3LYO/6-31G(d) optimization. The starting geometry for optimization was based on the X-ray structures of $\mathbf{B 3}$ of our previous work. ${ }^{161}$ The optimized structure of $\mathbf{B 3}^{\prime}$ is well agreement with the X-ray structure of B3, indicating the optimized geometries are quite reliable. Vibration analysis was performed at the same DFT-B3LYO/6-31G(d) level in order to check the stable minimum on the potential energy surface. 


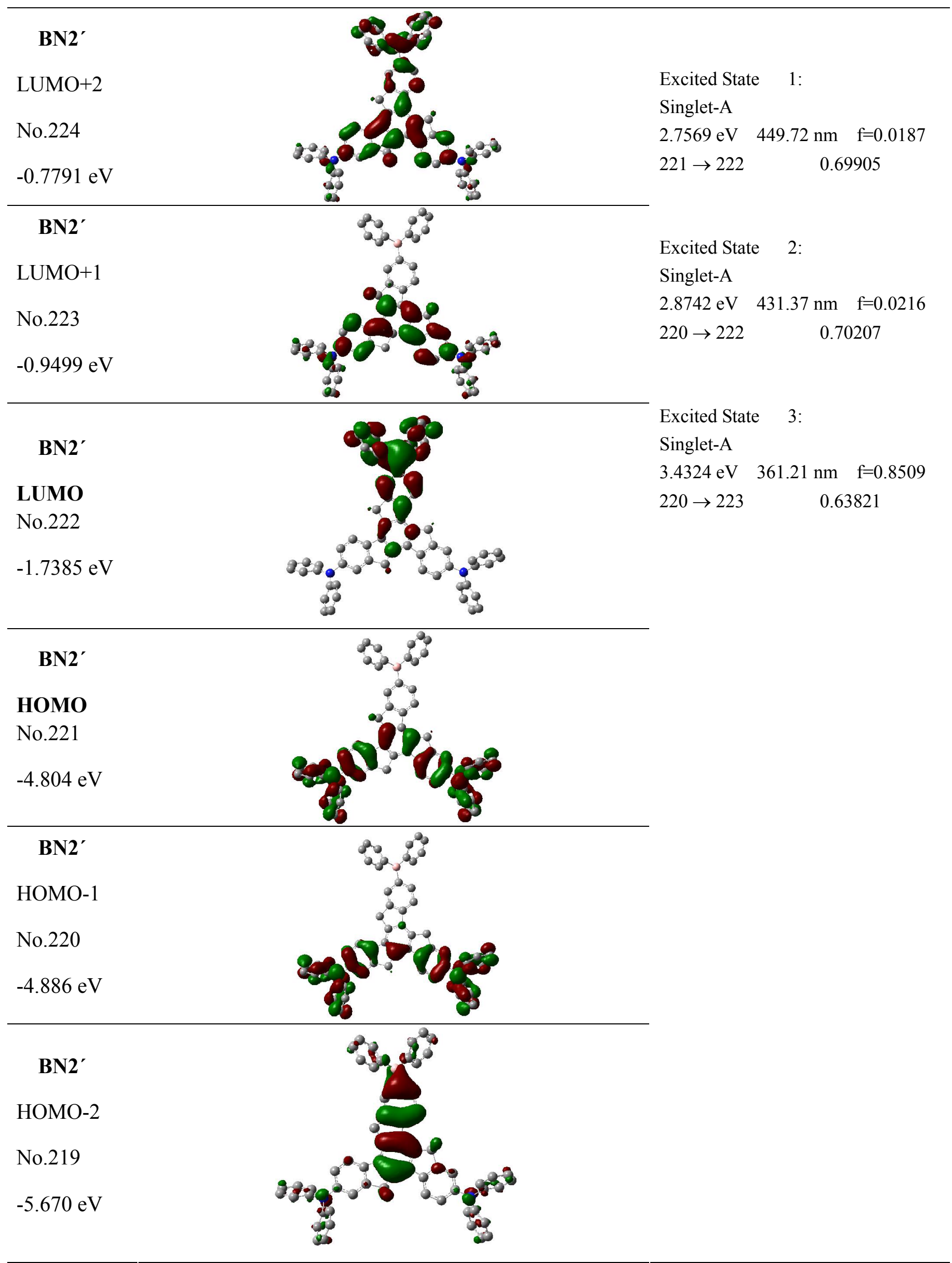




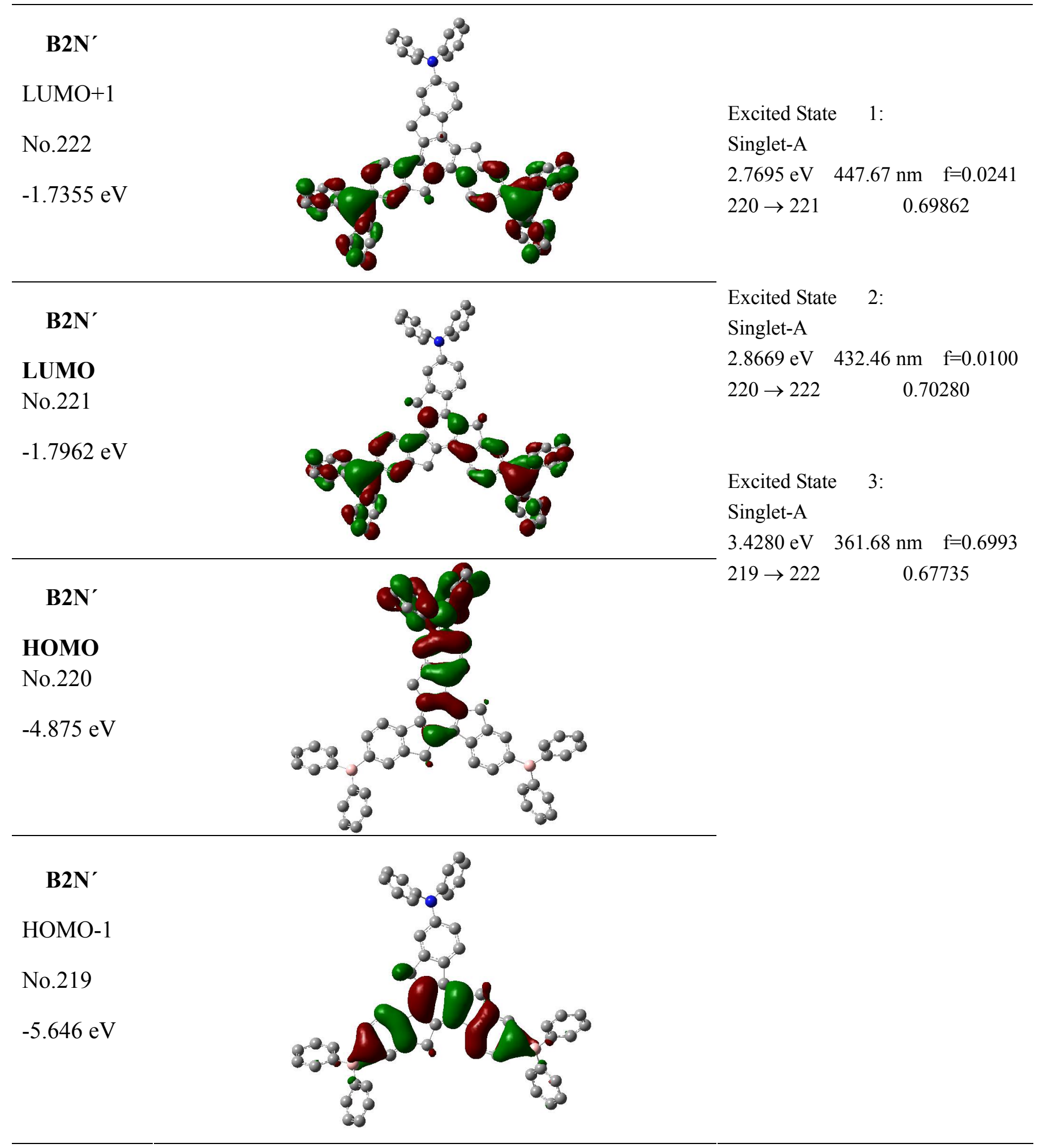




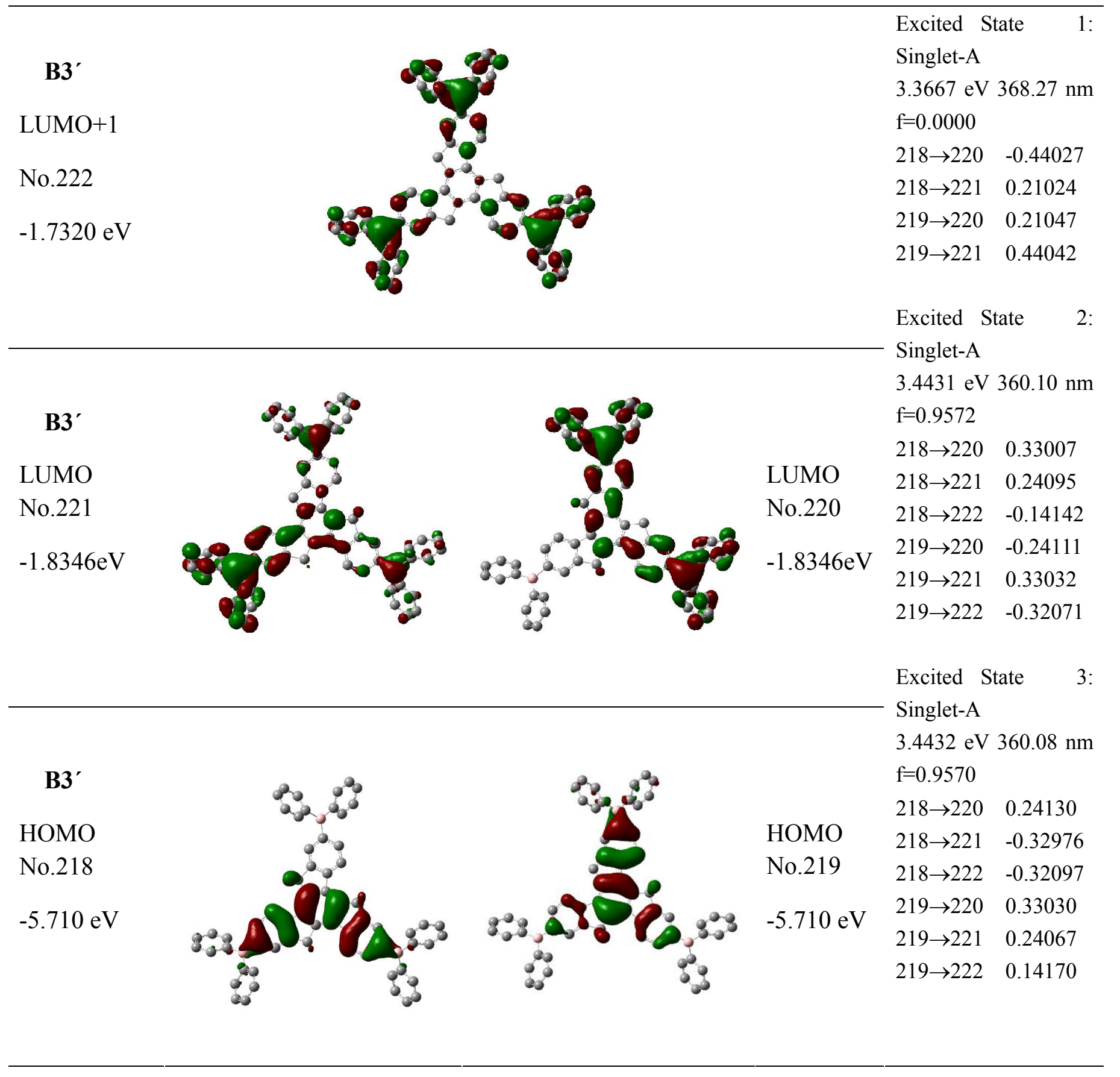




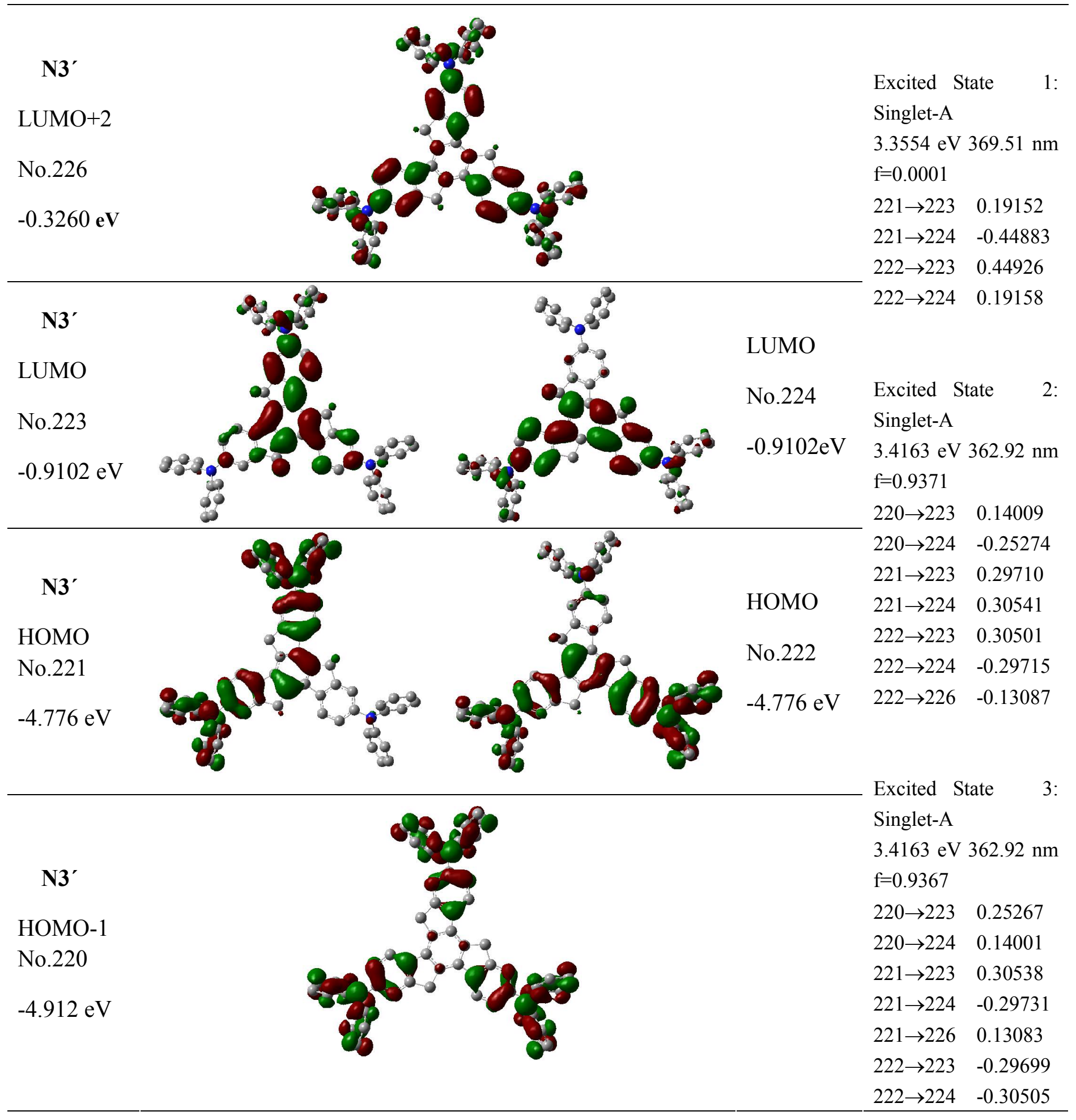

Figure S17 The graphic representations of the frontier molecular orbits of B3', N3', BN2' and $\mathbf{B} 2 \mathbf{N}^{\prime}$. 
The computed total energy of $\mathbf{B 2} \mathbf{N}^{\prime}$ is -2532.38 hartree $(-68906.06 \mathrm{eV})$.

The Z-matrix of $\mathbf{B 2} \mathbf{N}^{\prime}$ is as follows:

\begin{tabular}{|c|c|c|c|c|c|c|c|}
\hline C & 1 & B1 & & & & & \\
\hline $\mathrm{H}$ & 2 & B2 & 1 & $\mathrm{~A} 1$ & & & \\
\hline C & 2 & B3 & 1 & $\mathrm{~A} 2$ & 3 & D1 & 0 \\
\hline C & 4 & B4 & 2 & A3 & 1 & D2 & 0 \\
\hline $\mathrm{H}$ & 5 & B5 & 4 & A4 & 2 & D3 & 0 \\
\hline C & 5 & B6 & 4 & A5 & 2 & D4 & 0 \\
\hline $\mathrm{H}$ & 7 & B7 & 5 & A6 & 4 & D5 & 0 \\
\hline C & 7 & B8 & 5 & $\mathrm{~A} 7$ & 4 & D6 & 0 \\
\hline C & 9 & B9 & 7 & A8 & 5 & D7 & 0 \\
\hline C & 10 & B10 & 9 & A9 & 7 & D8 & 0 \\
\hline C & 1 & B11 & 2 & $\mathrm{~A} 10$ & 4 & D9 & 0 \\
\hline C & 4 & B12 & 2 & A11 & 1 & D10 & 0 \\
\hline C & 13 & B13 & 4 & $\mathrm{~A} 12$ & 2 & D11 & 0 \\
\hline C & 14 & B14 & 13 & $\mathrm{~A} 13$ & 4 & D12 & 0 \\
\hline $\mathrm{H}$ & 15 & B15 & 14 & $\mathrm{~A} 14$ & 13 & D13 & 0 \\
\hline C & 15 & B16 & 14 & A15 & 13 & D14 & 0 \\
\hline C & 17 & B17 & 15 & A16 & 14 & D15 & 0 \\
\hline $\mathrm{H}$ & 18 & B18 & 17 & $\mathrm{~A} 17$ & 15 & D16 & 0 \\
\hline C & 18 & B19 & 17 & A18 & 15 & D17 & 0 \\
\hline C & 13 & B20 & 4 & A19 & 2 & D18 & 0 \\
\hline C & 21 & B21 & 13 & A20 & 4 & D19 & 0 \\
\hline C & 22 & B22 & 21 & A21 & 13 & D20 & 0 \\
\hline $\mathrm{H}$ & 23 & B23 & 22 & A22 & 21 & D21 & 0 \\
\hline C & 23 & B24 & 22 & A23 & 21 & D22 & 0 \\
\hline C & 25 & B25 & 23 & A24 & 22 & D23 & 0 \\
\hline $\mathrm{H}$ & 26 & B26 & 25 & A25 & 23 & D24 & 0 \\
\hline C & 26 & B27 & 25 & A26 & 23 & D25 & 0 \\
\hline C & 12 & B28 & 1 & A27 & 2 & D26 & 0 \\
\hline C & 29 & B29 & 12 & A28 & 1 & D27 & 0 \\
\hline $\mathrm{H}$ & 30 & B30 & 29 & A29 & 12 & D28 & 0 \\
\hline C & 30 & B31 & 29 & $\mathrm{~A} 30$ & 12 & D29 & 0 \\
\hline C & 32 & B32 & 30 & A31 & 29 & D30 & 0 \\
\hline $\mathrm{H}$ & 33 & B33 & 32 & A32 & 30 & D31 & 0 \\
\hline C & 33 & B34 & 32 & A33 & 30 & D32 & 0 \\
\hline $\mathrm{H}$ & 35 & B35 & 33 & A34 & 32 & D33 & 0 \\
\hline C & 35 & B36 & 33 & A35 & 32 & D34 & 0 \\
\hline C & 37 & B37 & 35 & A36 & 33 & D35 & 0 \\
\hline C & 38 & B38 & 37 & A37 & 35 & D36 & 0 \\
\hline C & 29 & B39 & 12 & A38 & 1 & D37 & 0 \\
\hline C & 32 & B40 & 30 & A39 & 29 & D38 & 0 \\
\hline C & 41 & B41 & 32 & A40 & 30 & D39 & 0 \\
\hline
\end{tabular}




\begin{tabular}{|c|c|c|c|c|c|c|}
\hline C & 42 & B42 & 41 & A41 & 32 & D40 \\
\hline $\mathrm{H}$ & 43 & B43 & 42 & A42 & 41 & D41 \\
\hline C & 43 & B44 & 42 & A43 & 41 & D42 \\
\hline C & 45 & B45 & 43 & A44 & 42 & D43 \\
\hline $\mathrm{H}$ & 46 & B46 & 45 & A45 & 43 & D44 \\
\hline C & 46 & B47 & 45 & A46 & 43 & D45 \\
\hline C & 41 & B48 & 32 & A47 & 30 & D46 \\
\hline C & 49 & B49 & 41 & A48 & 32 & D47 \\
\hline C & 50 & B50 & 49 & A49 & 41 & D48 \\
\hline $\mathrm{H}$ & 51 & B51 & 50 & A50 & 49 & D49 \\
\hline C & 51 & B52 & 50 & A51 & 49 & D50 \\
\hline C & 53 & B53 & 51 & A52 & 50 & D51 \\
\hline $\mathrm{H}$ & 54 & B54 & 53 & A53 & 51 & D52 \\
\hline C & 54 & B55 & 53 & A54 & 51 & D53 \\
\hline B & 32 & B56 & 30 & A55 & 29 & D54 \\
\hline C & 11 & B57 & 10 & A56 & 9 & D55 \\
\hline C & 58 & B58 & 11 & A57 & 10 & D56 \\
\hline $\mathrm{H}$ & 59 & B59 & 58 & A58 & 11 & D57 \\
\hline C & 59 & B60 & 58 & A59 & 11 & D58 \\
\hline C & 61 & B61 & 59 & A60 & 58 & D59 \\
\hline $\mathrm{H}$ & 62 & B62 & 61 & A61 & 59 & D60 \\
\hline C & 62 & B63 & 61 & A62 & 59 & D61 \\
\hline $\mathrm{H}$ & 64 & B64 & 62 & A63 & 61 & D62 \\
\hline C & 64 & B65 & 62 & A64 & 61 & D63 \\
\hline C & 11 & B66 & 10 & A65 & 9 & D64 \\
\hline C & 67 & B67 & 11 & A66 & 10 & D65 \\
\hline C & 11 & B68 & 10 & A67 & 9 & D66 \\
\hline C & 61 & B69 & 59 & A68 & 58 & D67 \\
\hline C & 70 & B70 & 61 & A69 & 59 & D68 \\
\hline C & 71 & B71 & 70 & A70 & 61 & D69 \\
\hline $\mathrm{H}$ & 72 & B72 & 71 & A71 & 70 & D70 \\
\hline C & 72 & B73 & 71 & A72 & 70 & D71 \\
\hline C & 74 & B74 & 72 & A73 & 71 & D72 \\
\hline $\mathrm{H}$ & 75 & B75 & 74 & A74 & 72 & D73 \\
\hline C & 75 & B76 & 74 & A75 & 72 & D74 \\
\hline C & 70 & B77 & 61 & A76 & 59 & D75 \\
\hline C & 78 & B78 & 70 & A77 & 61 & D76 \\
\hline C & 79 & B79 & 78 & A78 & 70 & D77 \\
\hline $\mathrm{H}$ & 80 & B80 & 79 & A79 & 78 & D78 \\
\hline C & 80 & B81 & 79 & A80 & 78 & D79 \\
\hline C & 82 & B82 & 80 & A81 & 79 & D80 \\
\hline $\mathrm{H}$ & 83 & B83 & 82 & A82 & 80 & D81 \\
\hline C & 83 & B84 & 82 & A83 & 80 & D82 \\
\hline B & 61 & B85 & 59 & A84 & 58 & D83 \\
\hline
\end{tabular}




$\begin{array}{llllllll}\mathrm{H} & 74 & \mathrm{~B} 86 & 72 & \mathrm{~A} 85 & 71 & \mathrm{D} 84 & 0 \\ \mathrm{H} & 71 & \mathrm{~B} 87 & 70 & \mathrm{~A} 86 & 61 & \mathrm{D} 85 & 0 \\ \mathrm{H} & 77 & \mathrm{~B} 88 & 75 & \mathrm{~A} 87 & 74 & \mathrm{D} 86 & 0 \\ \mathrm{H} & 82 & \mathrm{~B} 89 & 80 & \mathrm{~A} 88 & 79 & \mathrm{D} 87 & 0 \\ \mathrm{H} & 85 & \mathrm{~B} 90 & 83 & \mathrm{~A} 89 & 82 & \mathrm{D} 88 & 0 \\ \mathrm{H} & 79 & \mathrm{~B} 91 & 78 & \mathrm{~A} 90 & 70 & \mathrm{D} 89 & 0 \\ \mathrm{H} & 69 & \mathrm{~B} 92 & 11 & \mathrm{~A} 91 & 10 & \mathrm{D} 90 & 0 \\ \mathrm{H} & 69 & \mathrm{~B} 93 & 11 & \mathrm{~A} 92 & 10 & \mathrm{D} 91 & 0 \\ \mathrm{H} & 25 & \mathrm{~B} 94 & 23 & \mathrm{~A} 93 & 22 & \mathrm{D} 92 & 0 \\ \mathrm{H} & 22 & \mathrm{~B} 95 & 21 & \mathrm{~A} 94 & 13 & \mathrm{D} 93 & 0 \\ \mathrm{H} & 28 & \mathrm{~B} 96 & 26 & \mathrm{~A} 95 & 25 & \mathrm{D} 94 & 0 \\ \mathrm{H} & 20 & \mathrm{~B} 97 & 18 & \mathrm{~A} 96 & 17 & \mathrm{D} 95 & 0 \\ \mathrm{H} & 14 & \mathrm{~B} 98 & 13 & \mathrm{~A} 97 & 4 & \mathrm{D} 96 & 0 \\ \mathrm{H} & 17 & \mathrm{~B} 99 & 15 & \mathrm{~A} 98 & 14 & \mathrm{D} 97 & 0 \\ \mathrm{H} & 53 & \mathrm{~B} 100 & 51 & \mathrm{~A} 99 & 50 & \mathrm{D} 98 & 0 \\ \mathrm{H} & 50 & \mathrm{~B} 101 & 49 & \mathrm{~A} 100 & 41 & \mathrm{D} 99 & 0 \\ \mathrm{H} & 56 & \mathrm{~B} 102 & 54 & \mathrm{~A} 101 & 53 & \mathrm{D} 100 & 0 \\ \mathrm{H} & 40 & \mathrm{~B} 103 & 29 & \mathrm{~A} 102 & 12 & \mathrm{D} 101 & 0 \\ \mathrm{H} & 40 & \mathrm{~B} 104 & 29 & \mathrm{~A} 103 & 12 & \mathrm{D} 102 & 0 \\ \mathrm{H} & 12 & \mathrm{~B} 105 & 1 & \mathrm{~A} 104 & 2 & \mathrm{D} 103 & 0 \\ \mathrm{H} & 12 & \mathrm{~B} 106 & 1 & \mathrm{~A} 105 & 2 & \mathrm{D} 104 & 0 \\ \mathrm{H} & 45 & \mathrm{~B} 107 & 43 & \mathrm{~A} 106 & 42 & \mathrm{D} 105 & 0 \\ \mathrm{H} & 48 & \mathrm{~B} 108 & 46 & \mathrm{~A} 107 & 45 & \mathrm{D} 106 & 0 \\ \mathrm{H} & \mathrm{B} 109 & 41 & \mathrm{~A} 108 & 32 & \mathrm{D} 107 & 0 \\ \mathrm{~N} & 4 & \mathrm{~B} 110 & 2 & \mathrm{~A} 109 & 1 & \mathrm{D} 108 & 0\end{array}$

Variables:

\begin{tabular}{llll} 
B1 & 1.38582 & B18 & 1.08693 \\
B2 & 1.08602 & B19 & 1.39358 \\
B3 & 1.40814 & B20 & 2.46157 \\
B4 & 1.40695 & B21 & 1.40445 \\
B5 & 1.08515 & B22 & 1.39355 \\
B6 & 1.39335 & B23 & 1.08693 \\
B7 & 1.08563 & B24 \\
B8 & 1.39843 & B25 & 1.3965 \\
B9 & 1.46847 & B26 \\
B10 & 1.39486 & B27 & 1.39645 \\
B11 & 1.51519 & B28 & 1.08693 \\
B12 & 2.46055 & B29 & 1.3935 \\
B13 & 1.40439 & B30 & 4.68229 \\
B14 & 1.39345 & B31 & 1.38599 \\
B15 & 1.08693 & B32 & 1.08733 \\
B16 & 1.39662 & B33 & 1.41637 \\
B17 & 1.39654 & B34 & 1.41489 \\
\hline
\end{tabular}




\begin{tabular}{|c|c|c|c|}
\hline B35 & 1.08575 & B79 & 1.39436 \\
\hline B36 & 1.39894 & B80 & 1.0872 \\
\hline B37 & 1.46872 & B81 & 1.39671 \\
\hline B38 & 1.39498 & B82 & 1.39665 \\
\hline B39 & 1.51525 & B83 & 1.08719 \\
\hline B40 & 2.71796 & B84 & 1.39435 \\
\hline B41 & 1.41224 & B85 & 1.56628 \\
\hline B42 & 1.39436 & B86 & 1.08726 \\
\hline B43 & 1.08721 & B87 & 1.08677 \\
\hline B44 & 1.39669 & B88 & 1.08672 \\
\hline B45 & 1.39663 & B89 & 1.08726 \\
\hline B46 & 1.08719 & B90 & 1.0867 \\
\hline B47 & 1.39435 & B91 & 1.08673 \\
\hline B48 & 2.71665 & B92 & 1.09955 \\
\hline B49 & 1.41225 & B93 & 1.09959 \\
\hline B50 & 1.3943 & B94 & 1.0863 \\
\hline B51 & 1.0872 & B95 & 1.08543 \\
\hline B52 & 1.3967 & B96 & 1.08537 \\
\hline B53 & 1.39668 & B97 & 1.08543 \\
\hline B54 & 1.0872 & B98 & 1.08546 \\
\hline B55 & 1.39435 & B99 & 1.0863 \\
\hline B56 & 1.56634 & B100 & 1.08727 \\
\hline B57 & 2.36793 & B101 & 1.08667 \\
\hline B58 & 1.38594 & B102 & 1.08672 \\
\hline B59 & 1.08733 & B103 & 1.09951 \\
\hline B60 & 1.41633 & B104 & 1.09949 \\
\hline B61 & 1.4149 & B105 & 1.09945 \\
\hline B62 & 1.08642 & B106 & 1.0993 \\
\hline B63 & 1.3939 & B107 & 1.08726 \\
\hline B64 & 1.08572 & B108 & 1.08667 \\
\hline B65 & 1.39904 & B109 & 1.08675 \\
\hline B66 & 1.41662 & B110 & 1.42029 \\
\hline B67 & 1.39473 & $\mathrm{~A} 1$ & 121.02512 \\
\hline B68 & 1.51332 & A2 & 119.40377 \\
\hline B69 & 2.71702 & A3 & 119.30722 \\
\hline B70 & 1.41217 & A4 & 119.04546 \\
\hline B71 & 1.39437 & A5 & 121.09753 \\
\hline B72 & 1.08721 & A6 & 119.29616 \\
\hline B73 & 1.39669 & A7 & 119.55976 \\
\hline B74 & 1.39672 & A8 & 131.99946 \\
\hline B75 & 1.0872 & A9 & 131.68034 \\
\hline B76 & 1.39437 & A10 & 128.67711 \\
\hline B77 & 2.7166 & A11 & 96.3033 \\
\hline B78 & 1.41221 & A12 & 97.06916 \\
\hline
\end{tabular}




\begin{tabular}{|c|c|c|c|}
\hline A13 & 120.31826 & A57 & 167.80299 \\
\hline A14 & 119.3256 & A58 & 119.94432 \\
\hline A15 & 120.56607 & A59 & 120.79878 \\
\hline A16 & 119.24272 & A60 & 117.24611 \\
\hline A17 & 120.09358 & A61 & 118.77824 \\
\hline A18 & 120.59998 & A62 & 122.51843 \\
\hline A19 & 60.06381 & A63 & 119.94921 \\
\hline A20 & 96.67269 & A64 & 118.96183 \\
\hline A21 & 120.31045 & A65 & 120.09639 \\
\hline A22 & 119.2827 & A66 & 120.02984 \\
\hline A23 & 120.61671 & A67 & 129.87185 \\
\hline A24 & 119.20261 & A68 & 95.18167 \\
\hline A25 & 120.10517 & A69 & 95.67767 \\
\hline A26 & 120.60394 & A70 & 121.7347 \\
\hline A27 & 145.42846 & A71 & 120.0073 \\
\hline A28 & 136.10593 & A72 & 119.93423 \\
\hline A29 & 119.90351 & A73 & 119.73402 \\
\hline A30 & 120.80851 & A74 & 120.05047 \\
\hline A31 & 117.22586 & A75 & 119.94911 \\
\hline A32 & 118.77545 & A76 & 60.0448 \\
\hline A33 & 122.53185 & A77 & 95.43439 \\
\hline A34 & 119.93554 & A78 & 121.71635 \\
\hline A35 & 118.97122 & A79 & 120.00083 \\
\hline A36 & 131.61205 & A80 & 119.94787 \\
\hline A37 & 131.55091 & A81 & 119.74377 \\
\hline A38 & 94.64215 & A82 & 120.05741 \\
\hline A39 & 95.16053 & A83 & 119.92991 \\
\hline A40 & 95.69812 & A84 & 121.41348 \\
\hline A41 & 121.73953 & A85 & 120.13509 \\
\hline A42 & 120.01141 & A86 & 119.11257 \\
\hline A43 & 119.93773 & A87 & 119.15577 \\
\hline A44 & 119.73616 & A88 & 120.12023 \\
\hline A45 & 120.05435 & A89 & 119.13253 \\
\hline A46 & 119.94351 & A90 & 119.11772 \\
\hline A47 & 60.02139 & A91 & 112.05831 \\
\hline A48 & 95.41567 & A92 & 112.03881 \\
\hline A49 & 121.73033 & A93 & 120.39681 \\
\hline A50 & 120.00641 & A94 & 119.41197 \\
\hline A51 & 119.93964 & A95 & 120.24633 \\
\hline A52 & 119.73949 & A96 & 120.31775 \\
\hline A53 & 120.05654 & A97 & 119.34369 \\
\hline A54 & 119.93899 & A98 & 120.38229 \\
\hline A55 & 121.45237 & A99 & 120.12934 \\
\hline A56 & 168.46705 & A100 & 119.11881 \\
\hline
\end{tabular}




\begin{tabular}{|c|c|c|c|}
\hline A101 & 119.15602 & D36 & 0.14322 \\
\hline A102 & 111.87564 & D37 & 0.35207 \\
\hline A103 & 111.98192 & D38 & -165.43988 \\
\hline A104 & 111.77956 & D39 & 55.05137 \\
\hline A105 & 111.97109 & D40 & -164.75132 \\
\hline A106 & 120.12804 & D41 & -178.92496 \\
\hline A107 & 119.13426 & D42 & 1.39571 \\
\hline A108 & 119.1139 & D43 & -0.69589 \\
\hline A109 & 120.3101 & D44 & 179.69832 \\
\hline D1 & -179.90864 & D45 & -0.66808 \\
\hline D2 & 0.29129 & D46 & -153.011 \\
\hline D3 & -179.46736 & D47 & -152.02467 \\
\hline D4 & 0.61004 & D48 & -164.48223 \\
\hline D5 & 179.92054 & D49 & -178.98927 \\
\hline D6 & -0.96295 & D50 & 1.39595 \\
\hline D7 & 179.9461 & D51 & -0.71304 \\
\hline D8 & 0.31787 & D52 & 179.68897 \\
\hline D9 & -179.78924 & D53 & -0.66068 \\
\hline D10 & -160.46705 & D54 & 179.18079 \\
\hline D11 & 70.91111 & D55 & 0.82161 \\
\hline D12 & -159.28606 & D56 & 2.07604 \\
\hline D13 & 179.99383 & D57 & -1.73296 \\
\hline D14 & -0.9654 & D58 & 179.15968 \\
\hline D15 & 0.41843 & D59 & -0.81016 \\
\hline D16 & 179.60612 & D60 & -179.83245 \\
\hline D17 & 0.52935 & D61 & -0.74686 \\
\hline D18 & -145.03005 & D62 & -178.85878 \\
\hline D19 & -145.19209 & D63 & 1.49893 \\
\hline D20 & -159.99481 & D64 & -179.49927 \\
\hline D21 & 179.9871 & D65 & -0.07236 \\
\hline D22 & -0.93806 & D66 & 0.45999 \\
\hline D23 & 0.49392 & D67 & -165.08826 \\
\hline D24 & 179.51803 & D68 & 55.49483 \\
\hline D25 & 0.44973 & D69 & -164.66643 \\
\hline D26 & 178.49345 & D70 & -178.91521 \\
\hline D27 & 179.90486 & D71 & 1.42941 \\
\hline D28 & -179.25856 & D72 & -0.70963 \\
\hline D29 & 1.5939 & D73 & 179.70085 \\
\hline D30 & -0.77509 & D74 & -0.68958 \\
\hline D31 & -179.73118 & D75 & -152.78252 \\
\hline D32 & -0.64499 & D76 & -152.06145 \\
\hline D33 & -178.90346 & D77 & -164.72088 \\
\hline D34 & 1.4296 & D78 & -178.92796 \\
\hline D35 & 179.11442 & D79 & 1.43043 \\
\hline
\end{tabular}




$\begin{array}{lrrr}\text { D80 } & -0.66319 & \text { D95 } & 179.1323 \\ \text { D81 } & 179.62782 & \text { D96 } & 20.68906 \\ \text { D82 } & -0.72496 & \text { D97 } & -179.53533 \\ \text { D83 } & 179.3385 & \text { D98 } & 179.29232 \\ \text { D84 } & 179.30561 & \text { D99 } & 16.27243 \\ \text { D85 } & 16.20559 & \text { D100 } & -179.45844 \\ \text { D86 } & -179.34196 & \text { D101 } & -120.23023 \\ \text { D87 } & 179.31212 & \text { D102 } & 120.70609 \\ \text { D88 } & -179.42589 & \text { D103 } & -60.68604 \\ \text { D89 } & 16.06954 & \text { D104 } & 58.3528 \\ \text { D90 } & 59.4514 & \text { D105 } & 179.32946 \\ \text { D91 } & -59.80959 & \text { D106 } & -179.39241 \\ \text { D92 } & -179.52431 & \text { D107 } & 16.1293 \\ \text { D93 } & 19.94784 & \text { D108 } & -179.71576 \\ \text { D94 } & 179.14976 & & \end{array}$

The computed total energy of BN2' is -2562.25 hartree $(-69718.82 \mathrm{eV})$. The Z-matrix of BN2' is as follows:

$\begin{array}{llllllll}\mathrm{C} & 1 & \mathrm{~B} 1 & & & & & \\ \mathrm{H} & 2 & \mathrm{~B} 2 & 1 & \mathrm{~A} 1 & & & \\ \mathrm{C} & 2 & \mathrm{~B} 3 & 1 & \mathrm{~A} 2 & 3 & \mathrm{D} 1 & 0 \\ \mathrm{C} & 4 & \mathrm{~B} 4 & 2 & \mathrm{~A} 3 & 1 & \mathrm{D} 2 & 0 \\ \mathrm{H} & 5 & \mathrm{~B} 5 & 4 & \mathrm{~A} 4 & 2 & \mathrm{D} 3 & 0 \\ \mathrm{C} & 5 & \mathrm{~B} 6 & 4 & \mathrm{~A} 5 & 2 & \mathrm{D} 4 & 0 \\ \mathrm{H} & 7 & \mathrm{~B} 7 & 5 & \mathrm{~A} 6 & 4 & \mathrm{D} 5 & 0 \\ \mathrm{C} & 7 & \mathrm{~B} 8 & 5 & \mathrm{~A} 7 & 4 & \mathrm{D} 6 & 0 \\ \mathrm{C} & 9 & \mathrm{~B} 9 & 7 & \mathrm{~A} 8 & 5 & \mathrm{D} 7 & 0 \\ \mathrm{C} & 10 & \mathrm{~B} 10 & 9 & \mathrm{~A} 9 & 7 & \mathrm{D} 8 & 0 \\ \mathrm{C} & 1 & \mathrm{~B} 11 & 2 & \mathrm{~A} 10 & 4 & \mathrm{D} 9 & 0 \\ \mathrm{C} & 4 & \mathrm{~B} 12 & 2 & \mathrm{~A} 11 & 1 & \mathrm{D} 10 & 0 \\ \mathrm{C} & 13 & \mathrm{~B} 13 & 4 & \mathrm{~A} 12 & 2 & \mathrm{D} 11 & 0 \\ \mathrm{C} & 14 & \mathrm{~B} 14 & 13 & \mathrm{~A} 13 & 4 & \mathrm{D} 12 & 0 \\ \mathrm{H} & 15 & \mathrm{~B} 15 & 14 & \mathrm{~A} 14 & 13 & \mathrm{D} 13 & 0 \\ \mathrm{C} & 15 & \mathrm{~B} 16 & 14 & \mathrm{~A} 15 & 13 & \mathrm{D} 14 & 0 \\ \mathrm{C} & 17 & \mathrm{~B} 17 & 15 & \mathrm{~A} 16 & 14 & \mathrm{D} 15 & 0 \\ \mathrm{H} & 18 & \mathrm{~B} 18 & 17 & \mathrm{~A} 17 & 15 & \mathrm{D} 16 & 0 \\ \mathrm{C} & 18 & \mathrm{~B} 19 & 17 & \mathrm{~A} 18 & 15 & \mathrm{D} 17 & 0 \\ \mathrm{C} & 13 & \mathrm{~B} 20 & 4 & \mathrm{~A} 19 & 2 & \mathrm{D} 18 & 0 \\ \mathrm{C} & 21 & \mathrm{~B} 21 & 13 & \mathrm{~A} 20 & 4 & \mathrm{D} 19 & 0 \\ \mathrm{C} & 22 & \mathrm{~B} 22 & 21 & \mathrm{~A} 21 & 13 & \mathrm{D} 20 & 0 \\ \mathrm{C} & 23 & \mathrm{~B} 23 & 22 & \mathrm{~A} 22 & 21 & \mathrm{D} 21 & 0 \\ \mathrm{C} & 23 & \mathrm{~B} 24 & 22 & \mathrm{~A} 23 & 21 & \mathrm{D} 22 & 0 \\ & 25 & \mathrm{~B} 25 & 23 & \mathrm{~A} 24 & 22 & \mathrm{D} 23 & 0 \\ \mathrm{C} & 26 & \mathrm{~B} 26 & 25 & \mathrm{~A} 25 & 23 & \mathrm{D} 24 & 0\end{array}$




\begin{tabular}{|c|c|c|c|c|c|c|}
\hline C & 26 & B27 & 25 & A26 & 23 & D25 \\
\hline C & 12 & B28 & 1 & A27 & 2 & D26 \\
\hline C & 29 & B29 & 12 & A28 & 1 & D27 \\
\hline $\mathrm{H}$ & 30 & B30 & 29 & A29 & 12 & D28 \\
\hline C & 30 & B31 & 29 & A30 & 12 & D29 \\
\hline C & 32 & B32 & 30 & A31 & 29 & D30 \\
\hline $\mathrm{H}$ & 33 & B33 & 32 & A32 & 30 & D31 \\
\hline C & 33 & B34 & 32 & A33 & 30 & D32 \\
\hline $\mathrm{H}$ & 35 & B35 & 33 & A34 & 32 & D33 \\
\hline C & 35 & B36 & 33 & A35 & 32 & D34 \\
\hline C & 37 & B37 & 35 & A36 & 33 & D35 \\
\hline C & 38 & B38 & 37 & A37 & 35 & D36 \\
\hline C & 29 & B39 & 12 & A38 & 1 & D37 \\
\hline C & 32 & B40 & 30 & A39 & 29 & D38 \\
\hline C & 41 & B41 & 32 & A40 & 30 & D39 \\
\hline C & 42 & B42 & 41 & A41 & 32 & D40 \\
\hline $\mathrm{H}$ & 43 & B43 & 42 & A42 & 41 & D41 \\
\hline C & 43 & B44 & 42 & A43 & 41 & D42 \\
\hline C & 45 & B45 & 43 & A44 & 42 & D43 \\
\hline $\mathrm{H}$ & 46 & B46 & 45 & A45 & 43 & D44 \\
\hline C & 46 & B47 & 45 & A46 & 43 & D45 \\
\hline C & 41 & B48 & 32 & A47 & 30 & D46 \\
\hline C & 49 & B49 & 41 & A48 & 32 & D47 \\
\hline C & 50 & B50 & 49 & A49 & 41 & D48 \\
\hline $\mathrm{H}$ & 51 & B51 & 50 & A50 & 49 & D49 \\
\hline C & 51 & B52 & 50 & A51 & 49 & D50 \\
\hline C & 53 & B53 & 51 & A52 & 50 & D51 \\
\hline $\mathrm{H}$ & 54 & B54 & 53 & A53 & 51 & D52 \\
\hline C & 54 & B55 & 53 & A54 & 51 & D53 \\
\hline B & 32 & B56 & 30 & A55 & 29 & D54 \\
\hline C & 11 & B57 & 10 & A56 & 9 & D55 \\
\hline C & 58 & B58 & 11 & A57 & 10 & D56 \\
\hline $\mathrm{H}$ & 59 & B59 & 58 & A58 & 11 & D57 \\
\hline C & 59 & B60 & 58 & A59 & 11 & D58 \\
\hline C & 61 & B61 & 59 & A60 & 58 & D59 \\
\hline $\mathrm{H}$ & 62 & B62 & 61 & A61 & 59 & D60 \\
\hline C & 62 & B63 & 61 & A62 & 59 & D61 \\
\hline $\mathrm{H}$ & 64 & B64 & 62 & A63 & 61 & D62 \\
\hline C & 64 & B65 & 62 & A64 & 61 & D63 \\
\hline C & 11 & B66 & 10 & A65 & 9 & D64 \\
\hline C & 67 & B67 & 11 & A66 & 10 & D65 \\
\hline C & 11 & B68 & 10 & A67 & 9 & D66 \\
\hline C & 61 & B69 & 59 & A68 & 58 & D67 \\
\hline C & 70 & B70 & 61 & A69 & 59 & D68 \\
\hline
\end{tabular}




\begin{tabular}{|c|c|c|c|c|c|c|}
\hline C & 71 & B71 & 70 & A70 & 61 & D69 \\
\hline $\mathrm{H}$ & 72 & B72 & 71 & A71 & 70 & D70 \\
\hline C & 72 & B73 & 71 & A72 & 70 & D71 \\
\hline C & 74 & B74 & 72 & A73 & 71 & D72 \\
\hline $\mathrm{H}$ & 75 & B75 & 74 & A74 & 72 & D73 \\
\hline C & 75 & B76 & 74 & A75 & 72 & D74 \\
\hline C & 70 & B77 & 61 & A76 & 59 & D75 \\
\hline C & 78 & B78 & 70 & A77 & 61 & D76 \\
\hline C & 79 & B79 & 78 & A78 & 70 & D77 \\
\hline $\mathrm{H}$ & 80 & B80 & 79 & A79 & 78 & D78 \\
\hline C & 80 & B81 & 79 & A80 & 78 & D79 \\
\hline C & 82 & B82 & 80 & A81 & 79 & D80 \\
\hline $\mathrm{H}$ & 83 & B83 & 82 & A82 & 80 & D81 \\
\hline C & 83 & B84 & 82 & A83 & 80 & D82 \\
\hline $\mathrm{H}$ & 74 & B85 & 72 & A84 & 71 & D83 \\
\hline $\mathrm{H}$ & 71 & B86 & 70 & A85 & 61 & D84 \\
\hline $\mathrm{H}$ & 77 & B87 & 75 & A86 & 74 & D85 \\
\hline $\mathrm{H}$ & 82 & B88 & 80 & A87 & 79 & D86 \\
\hline $\mathrm{H}$ & 85 & B89 & 83 & A88 & 82 & D87 \\
\hline $\mathrm{H}$ & 79 & B90 & 78 & A89 & 70 & D88 \\
\hline $\mathrm{H}$ & 69 & B91 & 11 & A90 & 10 & D89 \\
\hline $\mathrm{H}$ & 69 & B92 & 11 & A91 & 10 & D90 \\
\hline $\mathrm{H}$ & 25 & B93 & 23 & A92 & 22 & D91 \\
\hline $\mathrm{H}$ & 22 & B94 & 21 & A93 & 13 & D92 \\
\hline $\mathrm{H}$ & 28 & B95 & 26 & A94 & 25 & D93 \\
\hline $\mathrm{H}$ & 20 & B96 & 18 & A95 & 17 & D94 \\
\hline $\mathrm{H}$ & 14 & B97 & 13 & A96 & 4 & D95 \\
\hline $\mathrm{H}$ & 17 & B98 & 15 & A97 & 14 & D96 \\
\hline $\mathrm{H}$ & 53 & B99 & 51 & A98 & 50 & D97 \\
\hline $\mathrm{H}$ & 50 & B100 & 49 & A99 & 41 & D98 \\
\hline $\mathrm{H}$ & 56 & B101 & 54 & A100 & 53 & D99 \\
\hline $\mathrm{H}$ & 40 & B102 & 29 & A101 & 12 & D100 \\
\hline $\mathrm{H}$ & 40 & B103 & 29 & A102 & 12 & D101 \\
\hline $\mathrm{H}$ & 12 & B104 & 1 & A103 & 2 & D102 \\
\hline $\mathrm{H}$ & 12 & B105 & 1 & A104 & 2 & D103 \\
\hline $\mathrm{H}$ & 45 & B106 & 43 & A105 & 42 & D104 \\
\hline $\mathrm{H}$ & 48 & B107 & 46 & A106 & 45 & D105 \\
\hline $\mathrm{H}$ & 42 & B108 & 41 & A107 & 32 & D106 \\
\hline$N$ & 4 & B109 & 2 & A108 & 1 & D107 \\
\hline$N$ & 61 & B110 & 59 & A109 & 58 & D108 \\
\hline
\end{tabular}

Variables:

B1

B2
1.38584

1.08605
B3

B4
1.40809

1.40687 


\begin{tabular}{|c|c|c|c|}
\hline B5 & 1.08518 & B49 & 1.41226 \\
\hline B6 & 1.39339 & B50 & 1.39433 \\
\hline B7 & 1.08562 & B51 & 1.08721 \\
\hline B8 & 1.39846 & B52 & 1.39668 \\
\hline B9 & 1.46849 & B53 & 1.39666 \\
\hline B10 & 1.39438 & B54 & 1.08721 \\
\hline B11 & 1.51531 & B55 & 1.39439 \\
\hline B12 & 2.46117 & B56 & 1.5659 \\
\hline B13 & 1.4045 & B57 & 2.36887 \\
\hline B14 & 1.39352 & B58 & 1.38582 \\
\hline B15 & 1.08697 & B59 & 1.08602 \\
\hline B16 & 1.39653 & $\mathrm{~B} 60$ & 1.4081 \\
\hline B17 & 1.39656 & B61 & 1.40701 \\
\hline B18 & 1.08696 & B62 & 1.08514 \\
\hline B19 & 1.39357 & B63 & 1.39346 \\
\hline B20 & 2.46226 & B64 & 1.0856 \\
\hline B21 & 1.40453 & B65 & 1.39834 \\
\hline B22 & 1.39353 & B66 & 1.41658 \\
\hline B23 & 1.08698 & B67 & 1.39436 \\
\hline B24 & 1.39649 & B68 & 1.51413 \\
\hline B25 & 1.39652 & B69 & 2.46137 \\
\hline B26 & 1.08697 & B70 & 1.40446 \\
\hline B27 & 1.39352 & B71 & 1.39356 \\
\hline B28 & 4.68328 & B72 & 1.08698 \\
\hline B29 & 1.38595 & B73 & 1.39655 \\
\hline B30 & 1.08732 & B74 & 1.3965 \\
\hline B31 & 1.41664 & B75 & 1.08696 \\
\hline B32 & 1.41505 & B76 & 1.39357 \\
\hline B33 & 1.08637 & B77 & 2.46163 \\
\hline B34 & 1.39388 & B78 & 1.40444 \\
\hline B35 & 1.08579 & B79 & 1.39353 \\
\hline B36 & 1.39902 & B80 & 1.08696 \\
\hline B37 & 1.46849 & B81 & 1.39649 \\
\hline B38 & 1.39438 & B82 & 1.39656 \\
\hline B39 & 1.51537 & B83 & 1.08696 \\
\hline B40 & 2.71846 & B84 & 1.39348 \\
\hline B41 & 1.41211 & B85 & 1.08628 \\
\hline B42 & 1.39447 & B86 & 1.08536 \\
\hline B43 & 1.08722 & B87 & 1.08538 \\
\hline B44 & 1.39675 & B88 & 1.08628 \\
\hline B45 & 1.39662 & B89 & 1.08535 \\
\hline B46 & 1.0872 & B90 & 1.08534 \\
\hline B47 & 1.3943 & B91 & 1.0995 \\
\hline B48 & 2.71522 & B92 & 1.09959 \\
\hline
\end{tabular}




\begin{tabular}{|c|c|c|c|}
\hline B93 & 1.08627 & A27 & 145.35905 \\
\hline B94 & 1.08533 & A28 & 136.08581 \\
\hline B95 & 1.08534 & A29 & 119.87619 \\
\hline B96 & 1.08532 & A30 & 120.83739 \\
\hline B97 & 1.08538 & A31 & 117.18987 \\
\hline B98 & 1.08627 & A32 & 118.77979 \\
\hline B99 & 1.08727 & A33 & 122.54193 \\
\hline B100 & 1.08673 & A34 & 119.93356 \\
\hline B101 & 1.08666 & A35 & 118.98725 \\
\hline B102 & 1.09972 & A36 & 131.5956 \\
\hline B103 & 1.09959 & A37 & 131.52593 \\
\hline B104 & 1.09942 & A38 & 94.65113 \\
\hline B105 & 1.09935 & A39 & 94.99108 \\
\hline B106 & 1.08727 & A40 & 96.02278 \\
\hline B107 & 1.08672 & A41 & 121.72191 \\
\hline B108 & 1.08676 & A42 & 119.99723 \\
\hline B109 & 1.42062 & A43 & 119.95154 \\
\hline B110 & 1.42077 & A44 & 119.73044 \\
\hline A1 & 121.01557 & A45 & 120.05695 \\
\hline A2 & 119.41466 & A46 & 119.93382 \\
\hline A3 & 119.30329 & A47 & 60.05202 \\
\hline A4 & 119.042 & A48 & 95.34808 \\
\hline A5 & 121.09158 & A49 & 121.73822 \\
\hline A6 & 119.27995 & A50 & 120.00276 \\
\hline A7 & 119.57968 & A51 & 119.94102 \\
\hline A8 & 131.99952 & A52 & 119.73127 \\
\hline A9 & 131.66295 & A53 & 120.05255 \\
\hline A10 & 128.67915 & A54 & 119.94564 \\
\hline A11 & 96.47254 & A55 & 121.45155 \\
\hline A12 & 96.91184 & A56 & 168.41667 \\
\hline A13 & 120.3066 & A57 & 167.23305 \\
\hline A14 & 119.29891 & A58 & 121.00773 \\
\hline A15 & 120.59697 & A59 & 119.41409 \\
\hline A16 & 119.22408 & A60 & 119.30028 \\
\hline A17 & 120.10117 & A61 & 119.04737 \\
\hline A18 & 120.60078 & A62 & 121.09656 \\
\hline A19 & 60.02959 & A63 & 119.31017 \\
\hline A20 & 96.69021 & A64 & 119.57104 \\
\hline A21 & 120.31433 & A65 & 120.18211 \\
\hline A22 & 119.27343 & A66 & 119.89617 \\
\hline A23 & 120.61999 & A67 & 129.83863 \\
\hline A24 & 119.19348 & A68 & 96.42512 \\
\hline A25 & 120.10586 & A69 & 96.85142 \\
\hline A26 & 120.61637 & A70 & 120.31965 \\
\hline
\end{tabular}




\begin{tabular}{|c|c|c|c|}
\hline A71 & 119.28591 & D6 & -1.00945 \\
\hline A72 & 120.60635 & D7 & -179.86253 \\
\hline A73 & 119.20501 & D8 & 0.12828 \\
\hline A74 & 120.10516 & D9 & 179.99735 \\
\hline A75 & 120.60965 & D10 & -160.15477 \\
\hline A76 & 60.04869 & D11 & 70.73746 \\
\hline A77 & 96.7168 & D12 & -159.74614 \\
\hline A78 & 120.31616 & D13 & -179.96631 \\
\hline A79 & 119.28345 & D14 & -0.8836 \\
\hline A80 & 120.60428 & D15 & 0.45175 \\
\hline A81 & 119.20865 & D16 & 179.53448 \\
\hline A82 & 120.10167 & D17 & 0.43253 \\
\hline A83 & 120.6123 & D18 & -144.84839 \\
\hline A84 & 120.39713 & D19 & -145.0442 \\
\hline A85 & 119.38093 & D20 & -160.09431 \\
\hline A86 & 120.28524 & D21 & -179.99296 \\
\hline A87 & 120.39957 & D22 & -0.90027 \\
\hline A88 & 120.2929 & D23 & 0.46399 \\
\hline A89 & 119.37915 & D24 & 179.51405 \\
\hline A90 & 112.10781 & D25 & 0.42688 \\
\hline A91 & 112.08917 & D26 & 178.89914 \\
\hline A92 & 120.40611 & $\mathrm{D} 27$ & 179.52703 \\
\hline A93 & 119.41026 & D28 & -179.38357 \\
\hline A94 & 120.2887 & D29 & 1.52629 \\
\hline A95 & 120.31822 & D30 & -0.78525 \\
\hline A96 & 119.36986 & D31 & -179.64021 \\
\hline A97 & 120.38449 & D32 & -0.55983 \\
\hline A98 & 120.13522 & D33 & -178.95937 \\
\hline A99 & 119.12747 & D34 & 1.33281 \\
\hline A100 & 119.15352 & D35 & 179.20004 \\
\hline A101 & 111.84786 & D36 & 0.09107 \\
\hline A102 & 111.9171 & D37 & -0.01427 \\
\hline A103 & 111.71648 & D38 & -165.72356 \\
\hline A104 & 111.90849 & D39 & 54.77592 \\
\hline A105 & 120.12576 & D40 & -164.48418 \\
\hline A106 & 119.11855 & D41 & -178.94608 \\
\hline A107 & 119.09611 & D42 & 1.36714 \\
\hline A108 & 120.32615 & D43 & -0.71182 \\
\hline A109 & 120.30026 & D44 & 179.74737 \\
\hline D1 & -179.96037 & D45 & -0.65704 \\
\hline D2 & 0.33944 & D46 & -153.83012 \\
\hline D3 & -179.36656 & D47 & -151.88377 \\
\hline D4 & 0.6427 & D48 & -164.43355 \\
\hline D5 & 179.92757 & D49 & -178.94599 \\
\hline
\end{tabular}




\begin{tabular}{lrlr} 
D50 & 1.44396 & D80 & 0.44758 \\
D51 & -0.66347 & D81 & 179.53248 \\
D52 & 179.60843 & D82 & 0.43468 \\
D53 & -0.70143 & D83 & -179.55255 \\
D54 & 179.28065 & D84 & 20.25327 \\
D55 & -0.16252 & D85 & 179.0749 \\
D56 & -2.12926 & D86 & -179.5474 \\
D57 & 2.02331 & D87 & 179.11731 \\
D58 & -178.01746 & D88 & 20.04569 \\
D59 & 0.43773 & D89 & 59.87663 \\
D60 & -179.45277 & D90 & -59.45866 \\
D61 & 0.52026 & D91 & -179.54294 \\
D62 & 179.9575 & D92 & 19.92069 \\
D63 & -0.99611 & D93 & 179.12146 \\
D64 & -179.71496 & D94 & 179.15924 \\
D65 & -0.05731 & D95 & 20.28671 \\
D66 & 0.18309 & D96 & -179.55181 \\
D67 & -160.04724 & D97 & 179.28619 \\
D68 & 70.36598 & D98 & 16.34743 \\
D69 & -159.79208 & D99 & -179.51034 \\
D70 & -179.96367 & D100 & -120.22401 \\
D71 & -0.88941 & D101 & 120.76406 \\
D72 & 0.4196 & D102 & -60.4574 \\
D73 & 179.56315 & D103 & 58.50757 \\
D74 & 0.47987 & D104 & 179.34266 \\
D75 & -145.14183 & D105 & -179.30743 \\
D76 & -144.66297 & D106 & 16.40454 \\
D77 & -159.92633 & D107 & -179.60922 \\
D78 & -179.98887 & D108 & -179.44116 \\
D79 & -0.88814 & & \\
& & & \\
\hline
\end{tabular}

The computed total energy of B3' is -2502.51 hartree $(-68093.90 \mathrm{eV})$. The Z-matrix of B3' is as follows:

$$
\text { c }
$$

$\mathrm{H}$

2

C

$\mathrm{H}$

C

$\mathrm{H}$

C

C

C

$\begin{array}{lllllll}1 & \text { B1 } & & & & & \\ 2 & \text { B2 } & 1 & \text { A1 } & & & \\ 2 & \text { B3 } & 1 & \text { A2 } & 3 & \text { D1 } & 0 \\ 4 & \text { B4 } & 2 & \text { A3 } & 1 & \text { D2 } & 0 \\ 5 & \text { B5 } & 4 & \text { A4 } & 2 & \text { D3 } & 0 \\ 5 & \text { B6 } & 4 & \text { A5 } & 2 & \text { D4 } & 0 \\ 7 & \text { B7 } & 5 & \text { A6 } & 4 & \text { D5 } & 0 \\ 7 & \text { B8 } & 5 & \text { A7 } & 4 & \text { D6 } & 0 \\ 9 & \text { B9 } & 7 & \text { A8 } & 5 & \text { D7 } & 0 \\ 10 & \text { B10 } & 9 & \text { A9 } & 7 & \text { D8 } & 0\end{array}$




\begin{tabular}{|c|c|c|c|c|c|c|}
\hline C & 1 & B11 & 2 & A10 & 4 & D9 \\
\hline C & 4 & B12 & 2 & A11 & 1 & D10 \\
\hline C & 13 & B13 & 4 & $\mathrm{~A} 12$ & 2 & D11 \\
\hline C & 14 & B14 & 13 & $\mathrm{~A} 13$ & 4 & D12 \\
\hline $\mathrm{H}$ & 15 & B15 & 14 & A14 & 13 & D13 \\
\hline C & 15 & B16 & 14 & A15 & 13 & D14 \\
\hline C & 17 & B17 & 15 & A16 & 14 & D15 \\
\hline $\mathrm{H}$ & 18 & B18 & 17 & $\mathrm{~A} 17$ & 15 & D16 \\
\hline C & 18 & B19 & 17 & A18 & 15 & D17 \\
\hline C & 13 & B20 & 4 & A19 & 2 & D18 \\
\hline C & 21 & B21 & 13 & A20 & 4 & D19 \\
\hline C & 22 & B22 & 21 & A21 & 13 & D20 \\
\hline $\mathrm{H}$ & 23 & B23 & 22 & A22 & 21 & D21 \\
\hline C & 23 & B24 & 22 & A23 & 21 & D22 \\
\hline C & 25 & B25 & 23 & A24 & 22 & D23 \\
\hline $\mathrm{H}$ & 26 & B26 & 25 & A25 & 23 & D24 \\
\hline C & 26 & B27 & 25 & A26 & 23 & D25 \\
\hline B & 4 & B28 & 2 & A27 & 1 & D26 \\
\hline C & 12 & B29 & 1 & A28 & 2 & D27 \\
\hline C & 30 & B30 & 12 & A29 & 1 & D28 \\
\hline $\mathrm{H}$ & 31 & B31 & 30 & A30 & 12 & D29 \\
\hline C & 31 & B32 & 30 & A31 & 12 & D30 \\
\hline C & 33 & B33 & 31 & A32 & 30 & D31 \\
\hline $\mathrm{H}$ & 34 & B34 & 33 & A33 & 31 & D32 \\
\hline C & 34 & B35 & 33 & A34 & 31 & D33 \\
\hline $\mathrm{H}$ & 36 & B36 & 34 & A35 & 33 & D34 \\
\hline C & 36 & B37 & 34 & A36 & 33 & D35 \\
\hline C & 38 & B38 & 36 & A37 & 34 & D36 \\
\hline C & 39 & B39 & 38 & A38 & 36 & D37 \\
\hline C & 30 & B40 & 12 & A39 & 1 & D38 \\
\hline C & 33 & B41 & 31 & A40 & 30 & D39 \\
\hline C & 42 & B42 & 33 & A41 & 31 & D40 \\
\hline C & 43 & B43 & 42 & A42 & 33 & D41 \\
\hline $\mathrm{H}$ & 44 & B44 & 43 & A43 & 42 & D42 \\
\hline C & 44 & B45 & 43 & A44 & 42 & D43 \\
\hline C & 46 & B46 & 44 & A45 & 43 & D44 \\
\hline $\mathrm{H}$ & 47 & B47 & 46 & A46 & 44 & D45 \\
\hline C & 47 & B48 & 46 & A47 & 44 & D46 \\
\hline C & 42 & B49 & 33 & A48 & 31 & D47 \\
\hline C & 50 & B50 & 42 & A49 & 33 & D48 \\
\hline C & 51 & B51 & 50 & A50 & 42 & D49 \\
\hline $\mathrm{H}$ & 52 & B52 & 51 & A51 & 50 & D50 \\
\hline C & 52 & B53 & 51 & A52 & 50 & D51 \\
\hline C & 54 & B54 & 52 & A53 & 51 & D52 \\
\hline
\end{tabular}




\begin{tabular}{|c|c|c|c|c|c|c|}
\hline $\mathrm{H}$ & 55 & B55 & 54 & A54 & 52 & D53 \\
\hline C & 55 & B56 & 54 & A55 & 52 & D54 \\
\hline B & 33 & B57 & 31 & A56 & 30 & D55 \\
\hline C & 11 & B58 & 10 & A57 & 9 & D56 \\
\hline C & 59 & B59 & 11 & A58 & 10 & D57 \\
\hline $\mathrm{H}$ & 60 & B60 & 59 & A59 & 11 & D58 \\
\hline C & 60 & B61 & 59 & A60 & 11 & D59 \\
\hline C & 62 & B62 & 60 & A61 & 59 & D60 \\
\hline $\mathrm{H}$ & 63 & B63 & 62 & A62 & 60 & D61 \\
\hline C & 63 & B64 & 62 & A63 & 60 & D62 \\
\hline $\mathrm{H}$ & 65 & B65 & 63 & A64 & 62 & D63 \\
\hline C & 65 & B66 & 63 & A65 & 62 & D64 \\
\hline C & 11 & B67 & 10 & A66 & 9 & D65 \\
\hline C & 68 & B68 & 11 & A67 & 10 & D66 \\
\hline C & 11 & B69 & 10 & A68 & 9 & D67 \\
\hline C & 62 & B70 & 60 & A69 & 59 & D68 \\
\hline C & 71 & B71 & 62 & A70 & 60 & D69 \\
\hline C & 72 & B72 & 71 & A71 & 62 & D70 \\
\hline $\mathrm{H}$ & 73 & B73 & 72 & A72 & 71 & D71 \\
\hline C & 73 & B74 & 72 & A73 & 71 & D72 \\
\hline C & 75 & B75 & 73 & A74 & 72 & D73 \\
\hline $\mathrm{H}$ & 76 & B76 & 75 & A75 & 73 & D74 \\
\hline C & 76 & B77 & 75 & A76 & 73 & D75 \\
\hline C & 71 & B78 & 62 & A77 & 60 & D76 \\
\hline C & 79 & B79 & 71 & A78 & 62 & D77 \\
\hline C & 80 & B80 & 79 & A79 & 71 & D78 \\
\hline $\mathrm{H}$ & 81 & B81 & 80 & A80 & 79 & D79 \\
\hline C & 81 & B82 & 80 & A81 & 79 & D80 \\
\hline C & 83 & B83 & 81 & A82 & 80 & D81 \\
\hline $\mathrm{H}$ & 84 & B84 & 83 & A83 & 81 & D82 \\
\hline C & 84 & B85 & 83 & A84 & 81 & D83 \\
\hline B & 62 & B86 & 60 & A85 & 59 & D84 \\
\hline $\mathrm{H}$ & 75 & B87 & 73 & A86 & 72 & D85 \\
\hline $\mathrm{H}$ & 72 & B88 & 71 & A87 & 62 & D86 \\
\hline $\mathrm{H}$ & 78 & B89 & 76 & A88 & 75 & D87 \\
\hline $\mathrm{H}$ & 83 & B90 & 81 & A89 & 80 & D88 \\
\hline $\mathrm{H}$ & 86 & B91 & 84 & A90 & 83 & D89 \\
\hline $\mathrm{H}$ & 80 & B92 & 79 & A91 & 71 & D90 \\
\hline $\mathrm{H}$ & 70 & B93 & 11 & A92 & 10 & D91 \\
\hline $\mathrm{H}$ & 70 & B94 & 11 & A93 & 10 & D92 \\
\hline $\mathrm{H}$ & 25 & B95 & 23 & A94 & 22 & D93 \\
\hline $\mathrm{H}$ & 22 & B96 & 21 & A95 & 13 & D94 \\
\hline $\mathrm{H}$ & 28 & B97 & 26 & A96 & 25 & D95 \\
\hline $\mathrm{H}$ & 20 & B98 & 18 & A97 & 17 & D96 \\
\hline
\end{tabular}




$\begin{array}{llllllll}\mathrm{H} & 14 & \mathrm{~B} 99 & 13 & \mathrm{~A} 98 & 4 & \mathrm{D} 97 & 0 \\ \mathrm{H} & 17 & \mathrm{~B} 100 & 15 & \mathrm{~A} 99 & 14 & \mathrm{D} 98 & 0 \\ \mathrm{H} & 54 & \mathrm{~B} 101 & 52 & \mathrm{~A} 100 & 51 & \mathrm{D} 99 & 0 \\ \mathrm{H} & 51 & \mathrm{~B} 102 & 50 & \mathrm{~A} 101 & 42 & \mathrm{D} 100 & 0 \\ \mathrm{H} & 57 & \mathrm{~B} 103 & 55 & \mathrm{~A} 102 & 54 & \mathrm{D} 101 & 0 \\ \mathrm{H} & 41 & \mathrm{~B} 104 & 30 & \mathrm{~A} 103 & 12 & \mathrm{D} 102 & 0 \\ \mathrm{H} & 41 & \mathrm{~B} 105 & 30 & \mathrm{~A} 104 & 12 & \mathrm{D} 103 & 0 \\ \mathrm{H} & 12 & \mathrm{~B} 106 & 1 & \mathrm{~A} 105 & 2 & \mathrm{D} 104 & 0 \\ \mathrm{H} & 12 & \mathrm{~B} 107 & 1 & \mathrm{~A} 106 & 2 & \mathrm{D} 105 & 0 \\ \mathrm{H} & 46 & \mathrm{~B} 108 & 44 & \mathrm{~A} 107 & 43 & \mathrm{D} 106 & 0 \\ \mathrm{H} & 49 & \mathrm{~B} 109 & 47 & \mathrm{~A} 108 & 46 & \mathrm{D} 107 & 0 \\ \mathrm{H} & 43 & \mathrm{~B} 110 & 42 & \mathrm{~A} 109 & 33 & \mathrm{D} 108 & 0\end{array}$

B1

1.38608

B31

1.08726

1.08726

B32

1.41632

B33

1.41632

B3

1.41491

B34

1.41491

1.08636

1.08636

B35

1.39396

1.39396

B36

1.0857

1.0857

B37

1.3988

1.3988

B38

1.46862

1.46862

1.39545

1.39544

B39

1.51526

1.51526

2.71797

2.71797

B41

1.41221

1.41221

1.39436

1.39436

1.0872

1.0872

1.39672

1.39672

1.39667

1.39667

1.08718

1.08718

1.39432

B18

1.39432

2.7156

2.7156

1.41229

1.41229

1.39428

1.39428

1.08718

1.08718

1.39669

1.39669

1.39668

1.39667

1.08719

1.08719

1.39434

1.39434

1.5668

1.5668

2.36807

4.6824

1.38608

1.38608

1.08726 


\begin{tabular}{|c|c|c|c|}
\hline B61 & 1.41632 & B105 & 1.09938 \\
\hline B62 & 1.41491 & B106 & 1.09938 \\
\hline B63 & 1.08636 & B107 & 1.09938 \\
\hline B64 & 1.39396 & B108 & 1.08725 \\
\hline B65 & 1.0857 & B109 & 1.08669 \\
\hline B66 & 1.3988 & B110 & 1.08677 \\
\hline B67 & 1.41595 & A1 & 119.89188 \\
\hline B68 & 1.39545 & A2 & 120.81818 \\
\hline B69 & 1.51347 & A3 & 117.21768 \\
\hline B70 & 2.71797 & A4 & 118.79251 \\
\hline B71 & 1.41221 & A5 & 122.53415 \\
\hline B72 & 1.39436 & A6 & 119.93371 \\
\hline B73 & 1.0872 & A7 & 118.95918 \\
\hline B74 & 1.39672 & A8 & 131.60577 \\
\hline B75 & 1.39667 & A9 & 131.54963 \\
\hline B76 & 1.08718 & A10 & 129.27057 \\
\hline B77 & 1.39432 & A11 & 94.94449 \\
\hline B78 & 2.7156 & $\mathrm{~A} 12$ & 95.91511 \\
\hline B79 & 1.41229 & A13 & 121.72495 \\
\hline B80 & 1.39428 & A14 & 120.01207 \\
\hline B81 & 1.08718 & A15 & 119.93972 \\
\hline B82 & 1.39669 & A16 & 119.74206 \\
\hline B83 & 1.39667 & A17 & 120.05524 \\
\hline B84 & 1.08719 & A18 & 119.93661 \\
\hline B85 & 1.39434 & A19 & 60.05611 \\
\hline B86 & 1.5668 & A20 & 95.36546 \\
\hline B87 & 1.08725 & A21 & 121.74032 \\
\hline B88 & 1.08677 & A22 & 120.00252 \\
\hline B89 & 1.08669 & A23 & 119.93651 \\
\hline B90 & 1.08725 & A24 & 119.73686 \\
\hline B91 & 1.08672 & A25 & 120.05186 \\
\hline B92 & 1.08673 & A26 & 119.94555 \\
\hline B93 & 1.09938 & A27 & 121.39134 \\
\hline B94 & 1.09938 & A28 & 145.39061 \\
\hline B95 & 1.08725 & A29 & 136.11934 \\
\hline B96 & 1.08673 & A30 & 119.89189 \\
\hline B97 & 1.08672 & A31 & 120.81818 \\
\hline B98 & 1.08669 & A32 & 117.21768 \\
\hline B99 & 1.08677 & A33 & 118.79249 \\
\hline B100 & 1.08725 & A34 & 122.53414 \\
\hline B101 & 1.08725 & A35 & 119.93372 \\
\hline B102 & 1.08673 & A36 & 118.95919 \\
\hline B103 & 1.08672 & A37 & 131.60578 \\
\hline B104 & 1.09938 & A38 & 131.5496 \\
\hline
\end{tabular}




\begin{tabular}{|c|c|c|c|}
\hline A39 & 94.60911 & A83 & 120.05186 \\
\hline A40 & 94.94449 & A84 & 119.94554 \\
\hline A41 & 95.91511 & A85 & 121.39134 \\
\hline A42 & 121.72493 & A86 & 120.12505 \\
\hline A43 & 120.012 & A87 & 119.12822 \\
\hline A44 & 119.93971 & A88 & 119.16586 \\
\hline A45 & 119.7421 & A89 & 120.13482 \\
\hline A46 & 120.05529 & A90 & 119.12099 \\
\hline A47 & 119.93656 & A91 & 119.12161 \\
\hline A48 & 60.05608 & A92 & 112.06233 \\
\hline A49 & 95.36542 & A93 & 111.99276 \\
\hline A50 & 121.74031 & A94 & 120.13486 \\
\hline A51 & 120.00252 & A95 & 119.12161 \\
\hline A52 & 119.9365 & A96 & 119.12102 \\
\hline A53 & 119.73684 & A97 & 119.1659 \\
\hline A54 & 120.05183 & A98 & 119.12822 \\
\hline A55 & 119.94557 & A99 & 120.12504 \\
\hline A56 & 121.39134 & A100 & 120.13488 \\
\hline A57 & 168.58113 & A101 & 119.12166 \\
\hline A58 & 167.8061 & A102 & 119.121 \\
\hline A59 & 119.89188 & A103 & 111.89418 \\
\hline A60 & 120.81819 & A104 & 111.97216 \\
\hline A61 & 117.21766 & A105 & 111.97212 \\
\hline A62 & 118.79251 & A106 & 111.89417 \\
\hline A63 & 122.53414 & A107 & 120.125 \\
\hline A64 & 119.93368 & A108 & 119.16585 \\
\hline A65 & 118.95921 & A109 & 119.12823 \\
\hline A66 & 120.00537 & D1 & -179.13321 \\
\hline A67 & 119.99461 & D2 & -0.75132 \\
\hline A68 & 129.98617 & D3 & -179.70432 \\
\hline A69 & 94.9445 & D4 & -0.58421 \\
\hline A70 & 95.91512 & D5 & -178.98603 \\
\hline A71 & 121.72495 & D6 & 1.3295 \\
\hline A72 & 120.01206 & D7 & 179.13247 \\
\hline A73 & 119.9397 & D8 & 0.05476 \\
\hline A74 & 119.74208 & D9 & -179.03492 \\
\hline A75 & 120.05522 & D10 & -165.62083 \\
\hline A76 & 119.93659 & D11 & 54.91039 \\
\hline A77 & 60.0561 & D12 & -164.43372 \\
\hline A78 & 95.36545 & D13 & -178.94128 \\
\hline A79 & 121.7403 & D14 & 1.38193 \\
\hline A80 & 120.0025 & D15 & -0.72892 \\
\hline A81 & 119.93649 & D16 & 179.74951 \\
\hline A82 & 119.73688 & D17 & -0.65365 \\
\hline
\end{tabular}




\begin{tabular}{|c|c|c|c|}
\hline D18 & -153.55445 & D62 & -0.58421 \\
\hline D19 & -151.96453 & D63 & -178.98603 \\
\hline D20 & -164.51565 & D64 & 1.3295 \\
\hline D21 & -178.94453 & D65 & -179.93729 \\
\hline D22 & 1.42622 & D66 & 0.06285 \\
\hline D23 & -0.65506 & D67 & -0.07265 \\
\hline D24 & 179.61778 & D68 & -165.62083 \\
\hline D25 & -0.71379 & D69 & 54.9104 \\
\hline D26 & 179.31016 & D70 & -164.43371 \\
\hline D27 & -179.43774 & D71 & -178.94124 \\
\hline D28 & 179.31733 & D72 & 1.38189 \\
\hline D29 & -179.43891 & D73 & -0.7289 \\
\hline D30 & 1.42788 & D74 & 179.74944 \\
\hline D31 & -0.75132 & D75 & -0.65366 \\
\hline D32 & -179.70433 & D76 & -153.55447 \\
\hline D33 & -0.58421 & D77 & -151.96455 \\
\hline D34 & -178.98603 & D78 & -164.51566 \\
\hline D35 & 1.3295 & D79 & -178.94452 \\
\hline D36 & 179.13247 & $\mathrm{D} 80$ & 1.42626 \\
\hline D37 & 0.05476 & D81 & -0.65508 \\
\hline D38 & -0.32323 & D82 & 179.61778 \\
\hline D39 & -165.62084 & D83 & -0.71374 \\
\hline D40 & 54.9104 & D84 & 179.31016 \\
\hline D41 & -164.43373 & D85 & 179.32196 \\
\hline D42 & -178.94127 & D86 & 16.46873 \\
\hline D43 & 1.38189 & D87 & -179.30535 \\
\hline D44 & -0.72885 & D88 & 179.3097 \\
\hline D45 & 179.74948 & D89 & -179.49827 \\
\hline D46 & -0.65373 & D90 & 16.26776 \\
\hline D47 & -153.55446 & D91 & 59.51743 \\
\hline D48 & -151.96457 & D92 & -59.72606 \\
\hline D49 & -164.51567 & D93 & 179.30975 \\
\hline D50 & -178.94447 & D94 & 16.26778 \\
\hline D51 & 1.42626 & D95 & -179.49833 \\
\hline D52 & -0.65505 & D96 & -179.30532 \\
\hline D53 & 179.61775 & D97 & 16.46875 \\
\hline D54 & -0.71379 & D98 & 179.32193 \\
\hline D55 & 179.31016 & D99 & 179.30971 \\
\hline D56 & 0.05483 & D100 & 16.26784 \\
\hline D57 & 1.77775 & D101 & -179.49827 \\
\hline D58 & -1.45842 & D102 & -120.17642 \\
\hline D59 & 179.40837 & D103 & 120.7099 \\
\hline D60 & -0.75132 & D104 & -58.96826 \\
\hline D61 & -179.70433 & D105 & 60.14543 \\
\hline
\end{tabular}


$\begin{array}{lrrr}\text { D106 } & 179.32191 & \text { D108 } & 16.46874 \\ \text { D107 } & -179.30527 & & \end{array}$

The computed total energy of N3' is -2592.12 hartree $(-70531.59 \mathrm{eV})$.

The Z-matrix of $\mathbf{N 3}^{\prime}$ is as follows:

\begin{tabular}{|c|c|c|c|c|c|c|}
\hline C & 1 & B1 & & & & \\
\hline $\mathrm{H}$ & 2 & B2 & 1 & A1 & & \\
\hline C & 2 & B3 & 1 & A2 & 3 & D1 \\
\hline C & 4 & B4 & 2 & A3 & 1 & D2 \\
\hline $\mathrm{H}$ & 5 & B5 & 4 & A4 & 2 & D3 \\
\hline C & 5 & B6 & 4 & A5 & 2 & D4 \\
\hline $\mathrm{H}$ & 7 & B7 & 5 & A6 & 4 & D5 \\
\hline C & 7 & B8 & 5 & A7 & 4 & D6 \\
\hline C & 9 & B9 & 7 & A8 & 5 & D7 \\
\hline C & 10 & B10 & 9 & A9 & 7 & D8 \\
\hline C & 1 & B11 & 2 & A10 & 4 & D9 \\
\hline C & 4 & B12 & 2 & A11 & 1 & D10 \\
\hline C & 13 & B13 & 4 & A12 & 2 & D11 \\
\hline C & 14 & B14 & 13 & A13 & 4 & D12 \\
\hline $\mathrm{H}$ & 15 & B15 & 14 & $\mathrm{~A} 14$ & 13 & D13 \\
\hline C & 15 & B16 & 14 & A15 & 13 & D14 \\
\hline C & 17 & B17 & 15 & A16 & 14 & D15 \\
\hline $\mathrm{H}$ & 18 & B18 & 17 & A17 & 15 & D16 \\
\hline C & 18 & B19 & 17 & A18 & 15 & D17 \\
\hline C & 4 & B20 & 2 & A19 & 1 & D18 \\
\hline C & 21 & B21 & 4 & A20 & 2 & D19 \\
\hline C & 22 & B22 & 21 & A21 & 4 & D20 \\
\hline $\mathrm{H}$ & 23 & B23 & 22 & A22 & 21 & D21 \\
\hline C & 23 & B24 & 22 & A23 & 21 & D22 \\
\hline C & 25 & B25 & 23 & A24 & 22 & D23 \\
\hline $\mathrm{H}$ & 26 & B26 & 25 & A25 & 23 & D24 \\
\hline C & 26 & B27 & 25 & A26 & 23 & D25 \\
\hline C & 12 & B28 & 1 & A27 & 2 & D26 \\
\hline C & 29 & B29 & 12 & A28 & 1 & D27 \\
\hline $\mathrm{H}$ & 30 & B30 & 29 & A29 & 12 & D28 \\
\hline C & 30 & B31 & 29 & A30 & 12 & D29 \\
\hline$C$ & 32 & B32 & 30 & A31 & 29 & D30 \\
\hline $\mathrm{H}$ & 33 & B33 & 32 & A32 & 30 & D31 \\
\hline C & 33 & B34 & 32 & A33 & 30 & D32 \\
\hline $\mathrm{H}$ & 35 & B35 & 33 & A34 & 32 & D33 \\
\hline C & 35 & B36 & 33 & A35 & 32 & D34 \\
\hline C & 37 & B37 & 35 & A36 & 33 & D35 \\
\hline C & 38 & B38 & 37 & A37 & 35 & D36 \\
\hline
\end{tabular}




\begin{tabular}{|c|c|c|c|c|c|c|}
\hline C & 29 & B39 & 12 & A38 & 1 & D37 \\
\hline C & 32 & B40 & 30 & A39 & 29 & D38 \\
\hline C & 41 & B41 & 32 & A40 & 30 & D39 \\
\hline C & 42 & B42 & 41 & A41 & 32 & D40 \\
\hline $\mathrm{H}$ & 43 & B43 & 42 & A42 & 41 & D41 \\
\hline C & 43 & B44 & 42 & A43 & 41 & D42 \\
\hline C & 45 & B45 & 43 & A44 & 42 & D43 \\
\hline $\mathrm{H}$ & 46 & B46 & 45 & A45 & 43 & D44 \\
\hline C & 46 & B47 & 45 & A46 & 43 & D45 \\
\hline C & 32 & B48 & 30 & A47 & 29 & D46 \\
\hline C & 49 & B49 & 32 & A48 & 30 & D47 \\
\hline C & 50 & B50 & 49 & A49 & 32 & D48 \\
\hline $\mathrm{H}$ & 51 & B51 & 50 & A50 & 49 & D49 \\
\hline C & 51 & B52 & 50 & A51 & 49 & D50 \\
\hline C & 53 & B53 & 51 & A52 & 50 & D51 \\
\hline $\mathrm{H}$ & 54 & B54 & 53 & A53 & 51 & D52 \\
\hline C & 54 & B55 & 53 & A54 & 51 & D53 \\
\hline C & 11 & B56 & 10 & A55 & 9 & D54 \\
\hline C & 57 & B57 & 11 & A56 & 10 & D55 \\
\hline $\mathrm{H}$ & 58 & B58 & 57 & A57 & 11 & D56 \\
\hline C & 58 & B59 & 57 & A58 & 11 & D57 \\
\hline C & 60 & B60 & 58 & A59 & 57 & D58 \\
\hline $\mathrm{H}$ & 61 & B61 & 60 & A60 & 58 & D59 \\
\hline C & 61 & B62 & 60 & A61 & 58 & D60 \\
\hline $\mathrm{H}$ & 63 & B63 & 61 & A62 & 60 & D61 \\
\hline C & 63 & B64 & 61 & A63 & 60 & D62 \\
\hline C & 11 & B65 & 10 & A64 & 9 & D63 \\
\hline C & 66 & B66 & 11 & A65 & 10 & D64 \\
\hline C & 11 & B67 & 10 & A66 & 9 & D65 \\
\hline C & 60 & B68 & 58 & A67 & 57 & D66 \\
\hline C & 69 & B69 & 60 & A68 & 58 & D67 \\
\hline C & 70 & B70 & 69 & A69 & 60 & D68 \\
\hline $\mathrm{H}$ & 71 & B71 & 70 & A70 & 69 & D69 \\
\hline C & 71 & B72 & 70 & A71 & 69 & D70 \\
\hline C & 73 & B73 & 71 & A72 & 70 & D71 \\
\hline $\mathrm{H}$ & 74 & B74 & 73 & A73 & 71 & D72 \\
\hline C & 74 & B75 & 73 & A74 & 71 & D73 \\
\hline C & 60 & B76 & 58 & A75 & 57 & D74 \\
\hline C & 77 & B77 & 60 & A76 & 58 & D75 \\
\hline C & 78 & B78 & 77 & A77 & 60 & D76 \\
\hline $\mathrm{H}$ & 79 & B79 & 78 & A78 & 77 & D77 \\
\hline C & 79 & B80 & 78 & A79 & 77 & D78 \\
\hline C & 81 & B81 & 79 & A80 & 78 & D79 \\
\hline $\mathrm{H}$ & 82 & B82 & 81 & A81 & 79 & D80 \\
\hline
\end{tabular}




\begin{tabular}{|c|c|c|c|c|c|c|}
\hline C & 82 & B83 & 81 & A82 & 79 & D81 \\
\hline $\mathrm{H}$ & 73 & B84 & 71 & A83 & 70 & D82 \\
\hline $\mathrm{H}$ & 70 & B85 & 69 & A84 & 60 & D83 \\
\hline $\mathrm{H}$ & 76 & B86 & 74 & A85 & 73 & D84 \\
\hline $\mathrm{H}$ & 81 & B87 & 79 & A86 & 78 & D85 \\
\hline $\mathrm{H}$ & 84 & B88 & 82 & A87 & 81 & D86 \\
\hline $\mathrm{H}$ & 78 & B89 & 77 & A88 & 60 & D87 \\
\hline $\mathrm{H}$ & 68 & B90 & 11 & A89 & 10 & D88 \\
\hline $\mathrm{H}$ & 68 & B91 & 11 & A90 & 10 & D89 \\
\hline $\mathrm{H}$ & 25 & B92 & 23 & A91 & 22 & D90 \\
\hline $\mathrm{H}$ & 22 & B93 & 21 & A92 & 4 & D91 \\
\hline $\mathrm{H}$ & 28 & B94 & 26 & A93 & 25 & D92 \\
\hline $\mathrm{H}$ & 20 & B95 & 18 & A94 & 17 & D93 \\
\hline $\mathrm{H}$ & 14 & B96 & 13 & A95 & 4 & D94 \\
\hline $\mathrm{H}$ & 17 & B97 & 15 & A96 & 14 & D95 \\
\hline $\mathrm{H}$ & 53 & B98 & 51 & A97 & 50 & D96 \\
\hline $\mathrm{H}$ & 50 & B99 & 49 & A98 & 32 & D97 \\
\hline $\mathrm{H}$ & 56 & B100 & 54 & A99 & 53 & D98 \\
\hline $\mathrm{H}$ & 40 & B101 & 29 & A100 & 12 & D99 \\
\hline $\mathrm{H}$ & 40 & B102 & 29 & A101 & 12 & D100 \\
\hline $\mathrm{H}$ & 12 & B103 & 1 & A102 & 2 & D101 \\
\hline $\mathrm{H}$ & 12 & B104 & 1 & A103 & 2 & D102 \\
\hline $\mathrm{H}$ & 45 & B105 & 43 & A104 & 42 & D103 \\
\hline $\mathrm{H}$ & 48 & B106 & 46 & A105 & 45 & D104 \\
\hline $\mathrm{H}$ & 42 & B107 & 41 & A106 & 32 & D105 \\
\hline$N$ & 41 & B108 & 32 & A107 & 30 & D106 \\
\hline $\mathrm{N}$ & 13 & B109 & 4 & A108 & 2 & D107 \\
\hline $\mathrm{N}$ & 69 & B110 & 60 & A109 & 58 & D108 \\
\hline
\end{tabular}

Variables:

B1

B2

B3

B4

B5

B6

B7

B8

B9

B10

B11

B12

B13

B14

1.38601
1.08615
1.4079
1.40656
1.08522
1.39355
1.08565
1.3984
1.46849
1.39382
1.5154
2.46139
1.40466
1.39349

1.38601

B15

B16

B17

B18

B19

B20

B21

B22

B23

B24

B25

B26

B27

B28
1.087

1.39653

1.39652

1.087

1.39353

2.46174

1.40451

1.39356

1.08699

1.39652

1.39654

1.08698

1.39345

4.68236 


\begin{tabular}{|c|c|c|c|}
\hline B29 & 1.38601 & B73 & 1.39652 \\
\hline B30 & 1.08615 & B74 & 1.087 \\
\hline B31 & 1.4079 & B75 & 1.39353 \\
\hline B32 & 1.40656 & B76 & 2.46174 \\
\hline B33 & 1.08522 & B77 & 1.40451 \\
\hline B34 & 1.39355 & B78 & 1.39355 \\
\hline B35 & 1.08565 & B79 & 1.08699 \\
\hline B36 & 1.3984 & B80 & 1.39652 \\
\hline B37 & 1.46849 & B81 & 1.39654 \\
\hline B38 & 1.39382 & B82 & 1.08698 \\
\hline B39 & 1.51541 & B83 & 1.39345 \\
\hline B40 & 2.46139 & B84 & 1.08626 \\
\hline B41 & 1.40466 & B85 & 1.08533 \\
\hline B42 & 1.39349 & B86 & 1.08536 \\
\hline B43 & 1.087 & B87 & 1.08628 \\
\hline B44 & 1.39653 & B88 & 1.08538 \\
\hline B45 & 1.39652 & B89 & 1.08539 \\
\hline B46 & 1.087 & B90 & 1.09956 \\
\hline B47 & 1.39353 & B91 & 1.09962 \\
\hline B48 & 2.46174 & B92 & 1.08628 \\
\hline B49 & 1.40451 & B93 & 1.08539 \\
\hline B50 & 1.39356 & B94 & 1.08538 \\
\hline B51 & 1.08699 & B95 & 1.08536 \\
\hline B52 & 1.39652 & B96 & 1.08533 \\
\hline B53 & 1.39654 & B97 & 1.08626 \\
\hline B54 & 1.08698 & B98 & 1.08628 \\
\hline B55 & 1.39345 & B99 & 1.08539 \\
\hline B56 & 2.36876 & B100 & 1.08538 \\
\hline B57 & 1.38601 & B101 & 1.09962 \\
\hline B58 & 1.08615 & B102 & 1.09956 \\
\hline B59 & 1.4079 & B103 & 1.09956 \\
\hline B60 & 1.40656 & B104 & 1.09962 \\
\hline B61 & 1.08522 & B105 & 1.08626 \\
\hline B62 & 1.39355 & B106 & 1.08536 \\
\hline B63 & 1.08565 & B107 & 1.08533 \\
\hline B64 & 1.3984 & B108 & 1.42125 \\
\hline B65 & 1.41691 & B109 & 1.42125 \\
\hline B66 & 1.39381 & B110 & 1.42125 \\
\hline B67 & 1.5143 & $\mathrm{~A} 1$ & 121.05796 \\
\hline B68 & 2.46139 & A2 & 119.40746 \\
\hline B69 & 1.40466 & A3 & 119.36711 \\
\hline B70 & 1.39349 & A4 & 119.01383 \\
\hline B71 & 1.087 & A5 & 121.05515 \\
\hline B72 & 1.39653 & A6 & 119.3309 \\
\hline
\end{tabular}




\begin{tabular}{|c|c|c|c|}
\hline A7 & 119.5623 & A51 & 120.62104 \\
\hline A8 & 131.90795 & A52 & 119.19715 \\
\hline A9 & 131.63595 & A53 & 120.10553 \\
\hline A10 & 128.7487 & A54 & 120.60896 \\
\hline A11 & 96.83959 & A55 & 168.5496 \\
\hline A12 & 96.57347 & A56 & 167.29396 \\
\hline A13 & 120.33278 & A57 & 121.05791 \\
\hline A14 & 119.27288 & A58 & 119.40748 \\
\hline A15 & 120.61968 & A59 & 119.36714 \\
\hline A16 & 119.18077 & A60 & 119.01385 \\
\hline A17 & 120.09859 & A61 & 121.05512 \\
\hline A18 & 120.63172 & A62 & 119.33086 \\
\hline A19 & 139.05568 & A63 & 119.56227 \\
\hline A20 & 139.51062 & A64 & 120.0376 \\
\hline A21 & 120.30978 & A65 & 119.96231 \\
\hline A22 & 119.27284 & A66 & 129.96337 \\
\hline A23 & 120.62104 & A67 & 96.8396 \\
\hline A24 & 119.19713 & A68 & 96.57348 \\
\hline A25 & 120.10556 & A69 & 120.33279 \\
\hline A26 & 120.60899 & A70 & 119.27291 \\
\hline A27 & 145.37735 & A71 & 120.6197 \\
\hline A28 & 136.62694 & A72 & 119.18073 \\
\hline A29 & 121.05794 & A73 & 120.09857 \\
\hline A30 & 119.40747 & A74 & 120.63175 \\
\hline A31 & 119.36714 & A75 & 139.05568 \\
\hline A32 & 119.01387 & A76 & 139.51064 \\
\hline A33 & 121.0551 & A77 & 120.30977 \\
\hline A34 & 119.33085 & A78 & 119.27279 \\
\hline A35 & 119.56231 & A79 & 120.62104 \\
\hline A36 & 131.90797 & A80 & 119.19717 \\
\hline A37 & 131.63592 & A81 & 120.10556 \\
\hline A38 & 94.62258 & A82 & 120.60897 \\
\hline A39 & 96.83962 & A83 & 120.41074 \\
\hline A40 & 96.57345 & A84 & 119.37529 \\
\hline A41 & 120.33278 & A85 & 120.27183 \\
\hline A42 & 119.2729 & A86 & 120.40094 \\
\hline A43 & 120.6197 & A87 & 120.2998 \\
\hline A44 & 119.18072 & A88 & 119.40942 \\
\hline A45 & 120.09855 & A89 & 112.1394 \\
\hline A46 & 120.63176 & A90 & 112.18652 \\
\hline A47 & 139.05568 & A91 & 120.401 \\
\hline A48 & 139.51063 & A92 & 119.40949 \\
\hline A49 & 120.30976 & A93 & 120.29981 \\
\hline A50 & 119.27281 & A94 & 120.27181 \\
\hline
\end{tabular}




\begin{tabular}{|c|c|c|c|}
\hline A95 & 119.37531 & D30 & 0.48688 \\
\hline A96 & 120.41076 & D31 & -179.56955 \\
\hline A97 & 120.40096 & D32 & 0.35794 \\
\hline A98 & 119.40952 & D33 & -179.9424 \\
\hline A99 & 120.29977 & D34 & -0.89006 \\
\hline A100 & 111.86744 & D35 & -179.63592 \\
\hline A101 & 111.73279 & D36 & 0.61588 \\
\hline A102 & 111.73281 & D37 & -0.17025 \\
\hline A103 & 111.86749 & D38 & -159.46559 \\
\hline A104 & 120.41075 & D39 & 70.82007 \\
\hline A105 & 120.27182 & D40 & -160.07633 \\
\hline A106 & 119.37531 & D41 & -179.97508 \\
\hline A107 & 30.02597 & D42 & -0.8803 \\
\hline A108 & 30.02599 & D43 & 0.39302 \\
\hline A109 & 30.026 & D44 & 179.56549 \\
\hline D1 & 179.96448 & D45 & 0.48959 \\
\hline D2 & 0.48688 & D46 & 149.53653 \\
\hline D3 & -179.56955 & D47 & 125.39473 \\
\hline D4 & 0.35794 & D48 & 149.33451 \\
\hline D5 & -179.9424 & D49 & -179.97748 \\
\hline D6 & -0.89006 & D50 & -0.87798 \\
\hline D7 & -179.63592 & D51 & 0.42373 \\
\hline D8 & 0.61588 & D52 & 179.55598 \\
\hline D9 & -179.93463 & D53 & 0.43366 \\
\hline D10 & -159.46559 & D54 & 0.72654 \\
\hline D11 & 70.82009 & D55 & -2.88902 \\
\hline D12 & -160.07635 & D56 & 1.93052 \\
\hline D13 & -179.97513 & D57 & -178.105 \\
\hline D14 & -0.88034 & D58 & 0.48688 \\
\hline D15 & 0.39303 & D59 & -179.56955 \\
\hline D16 & 179.56546 & D60 & 0.35794 \\
\hline D17 & 0.4896 & D61 & -179.9424 \\
\hline D18 & 149.53653 & D62 & -0.89006 \\
\hline D19 & 125.39477 & D63 & -179.98759 \\
\hline D20 & 149.33453 & D64 & -0.13001 \\
\hline D21 & -179.97747 & D65 & 0.23889 \\
\hline D22 & -0.87808 & D66 & -159.46559 \\
\hline D23 & 0.42381 & D67 & 70.8201 \\
\hline D24 & 179.55597 & D68 & -160.07632 \\
\hline D25 & 0.4336 & D69 & -179.97515 \\
\hline D26 & 179.70169 & D70 & -0.88034 \\
\hline D27 & -179.68766 & D71 & 0.39305 \\
\hline D28 & 179.48411 & D72 & 179.56554 \\
\hline D29 & -0.5514 & D73 & 0.48954 \\
\hline
\end{tabular}




$\begin{array}{lrrr}\text { D74 } & 149.53654 & \text { D92 } & 179.17581 \\ \text { D75 } & 125.39476 & \text { D93 } & 179.08178 \\ \text { D76 } & 149.33451 & \text { D94 } & 19.94747 \\ \text { D77 } & -179.97748 & \text { D95 } & -179.56796 \\ \text { D78 } & -0.87805 & \text { D96 } & -179.5661 \\ \text { D79 } & 0.42378 & \text { D97 } & -30.66303 \\ \text { D80 } & 179.55593 & \text { D98 } & 179.17585 \\ \text { D81 } & 0.43362 & \text { D99 } & -120.8174 \\ \text { D82 } & -179.56796 & \text { D100 } & 120.25264 \\ \text { D83 } & 19.94748 & \text { D101 } & -60.17229 \\ \text { D84 } & 179.08181 & \text { D102 } & 58.75766 \\ \text { D85 } & -179.56613 & \text { D103 } & -179.56795 \\ \text { D86 } & 179.17582 & \text { D104 } & 179.08177 \\ \text { D87 } & -30.66308 & \text { D105 } & 19.94749 \\ \text { D88 } & 59.65132 & \text { D106 } & -143.91136 \\ \text { D89 } & -59.77773 & \text { D107 } & -143.91138 \\ \text { D90 } & -179.56608 & \text { D108 } & -143.91141 \\ \text { D91 } & -30.66312 & & \end{array}$

(26) Gaussian 03, Revision B.05, M. J. Frisch, G. W. Trucks, H. B. Schlegel, G. E. Scuseria, M. A. Robb, J. R. Cheeseman, J. A. Montgomery, Jr., T. Vreven, K. N. Kudin, J. C. Burant, J. M. Millam, S. S. Iyengar, J. Tomasi, V. Barone, B. Mennucci, M. Cossi, G. Scalmani, N. Rega, G. A. Petersson, H. Nakatsuji, M. Hada, M. Ehara, K. Toyota, R. Fukuda, J. Hasegawa, M. Ishida, T. Nakajima, Y. Honda, O. Kitao, H. Nakai, M. Klene, X. Li, J. E. Knox, H. P. Hratchian, J. B. Cross, C. Adamo, J. Jaramillo, R. Gomperts, R. E. Stratmann, O. Yazyev, A. J. Austin, R. Cammi, C. Pomelli, J. W. Ochterski, P. Y. Ayala, K. Morokuma, G. A. Voth, P. Salvador, J. J. Dannenberg, V. G. Zakrzewski, S. Dapprich, A. D. Daniels, M. C. Strain, O. Farkas, D. K. Malick, A. D. Rabuck, K. Raghavachari, J. B. Foresman, J. V. Ortiz, Q. Cui, A. G. Baboul, S. Clifford, J. Cioslowski, B. B. Stefanov, G. Liu, A. Liashenko, P. Piskorz, I. Komaromi, R. L. Martin, D. J. Fox, T. Keith, M. A. Al-Laham, C. Y. Peng, A. Nanayakkara, Challacombe, P. M. W. Gill, B. Johnson, W. Chen, M. W. Wong, C. Gonzalez, and J. A. Pople, Gaussian, Inc., Pittsburgh PA, 2003. 
7. ${ }^{13} \mathrm{C}$ NMR
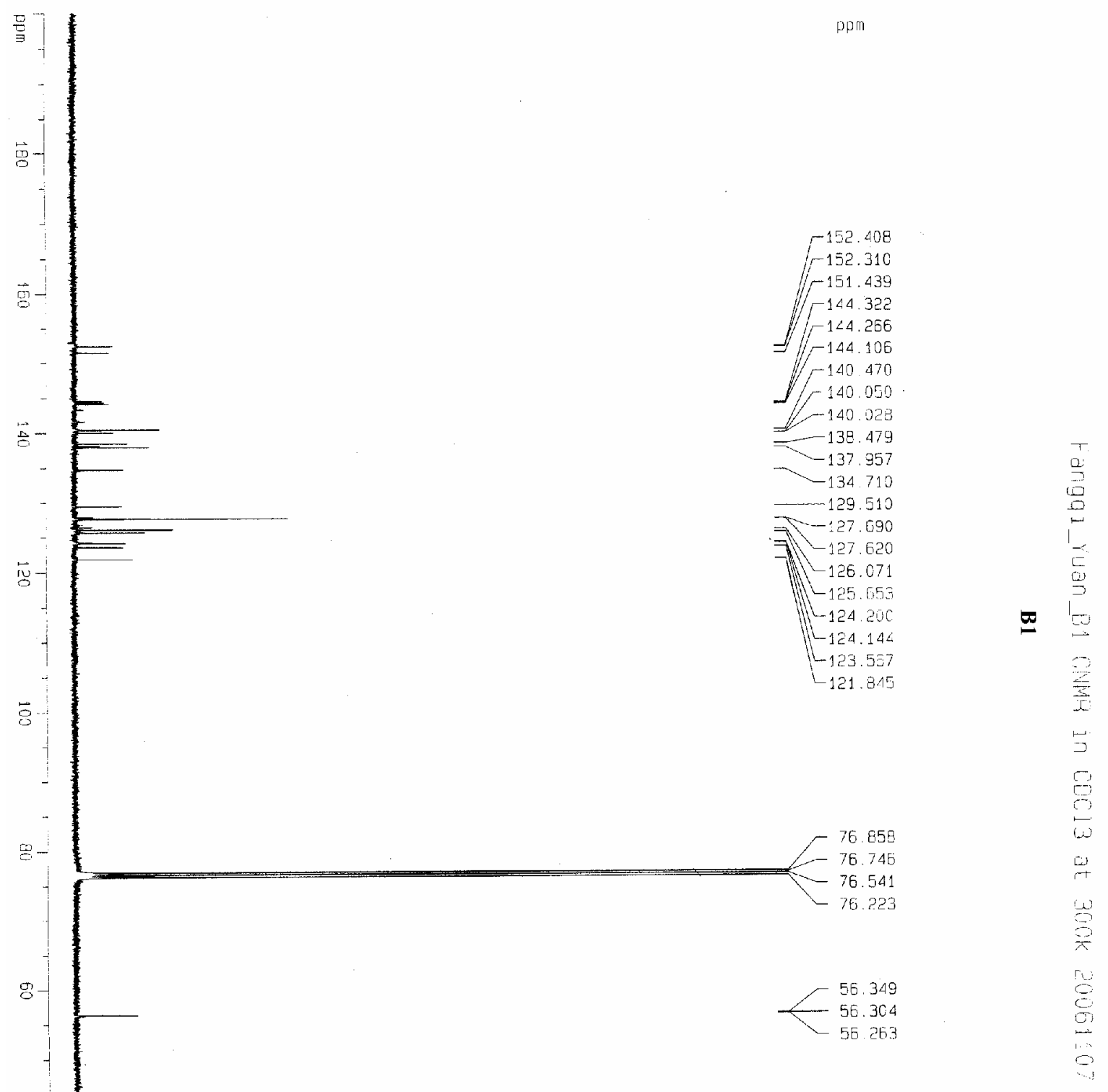

S46 


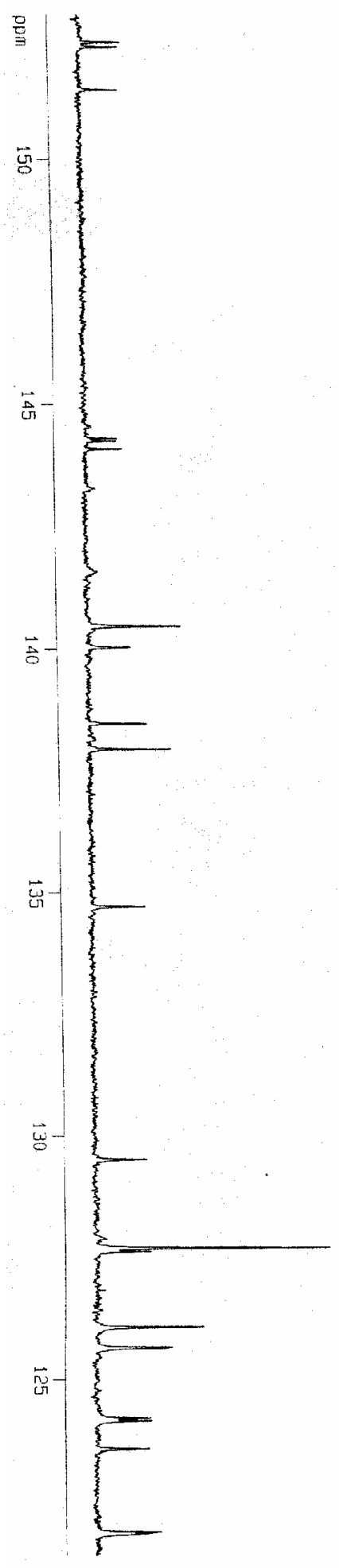

ppm

$-152.408$

$-152.310$

$-151.439$

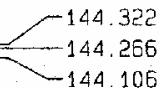

-140.470
-140.050

0
0
0

$-138.479$

137.957

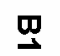

E

$\frac{5}{5}$

五

$-134.710$

9

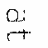

定

$-129.510$

0
8
0
0

$-\quad-127.690$
-127.620

$-126.071$

$-125.653$

$-124.200$

$-124.144$

$-123.567$

$-121.845$ 


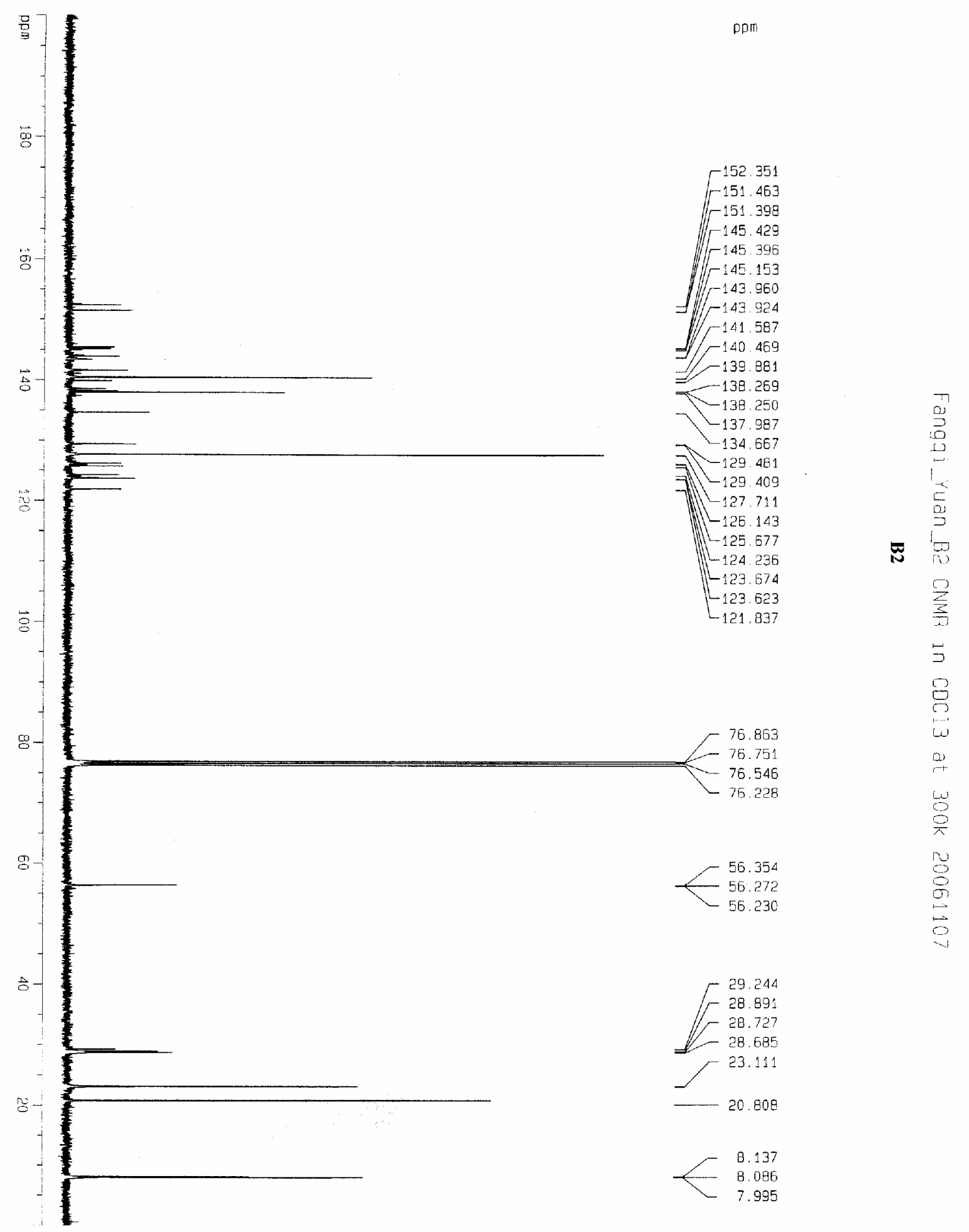




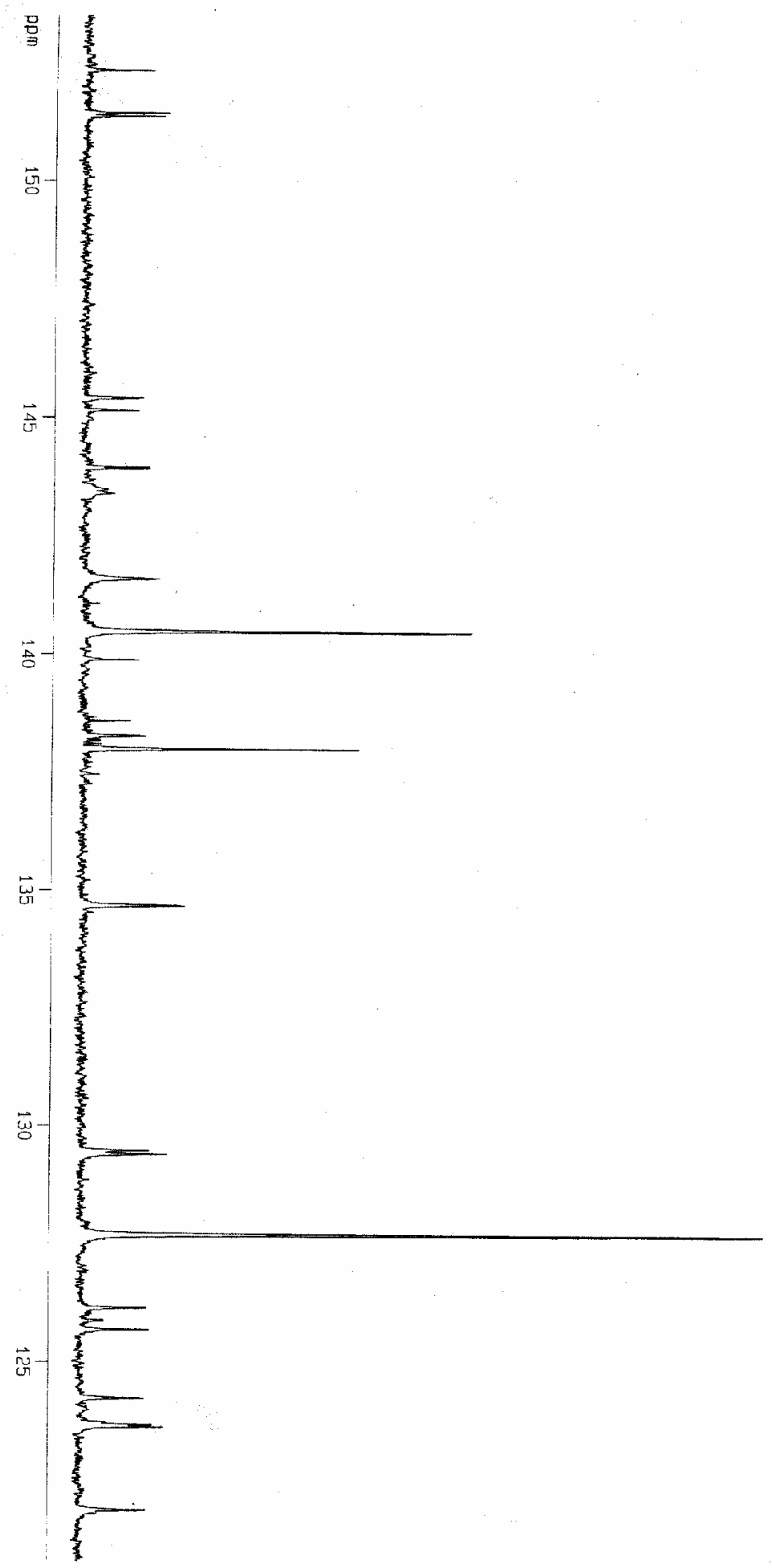

ppm

152. 351

$-151.463$

$-151.398$

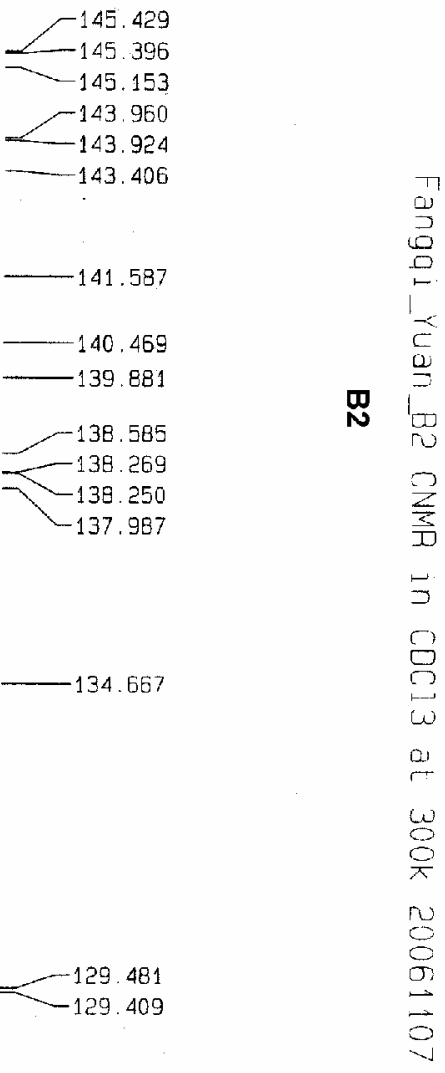

$-127.711$

$-126.143$

125.878

$-125.677$

$-124.236$

123.674
123.623

$-121.837$ 


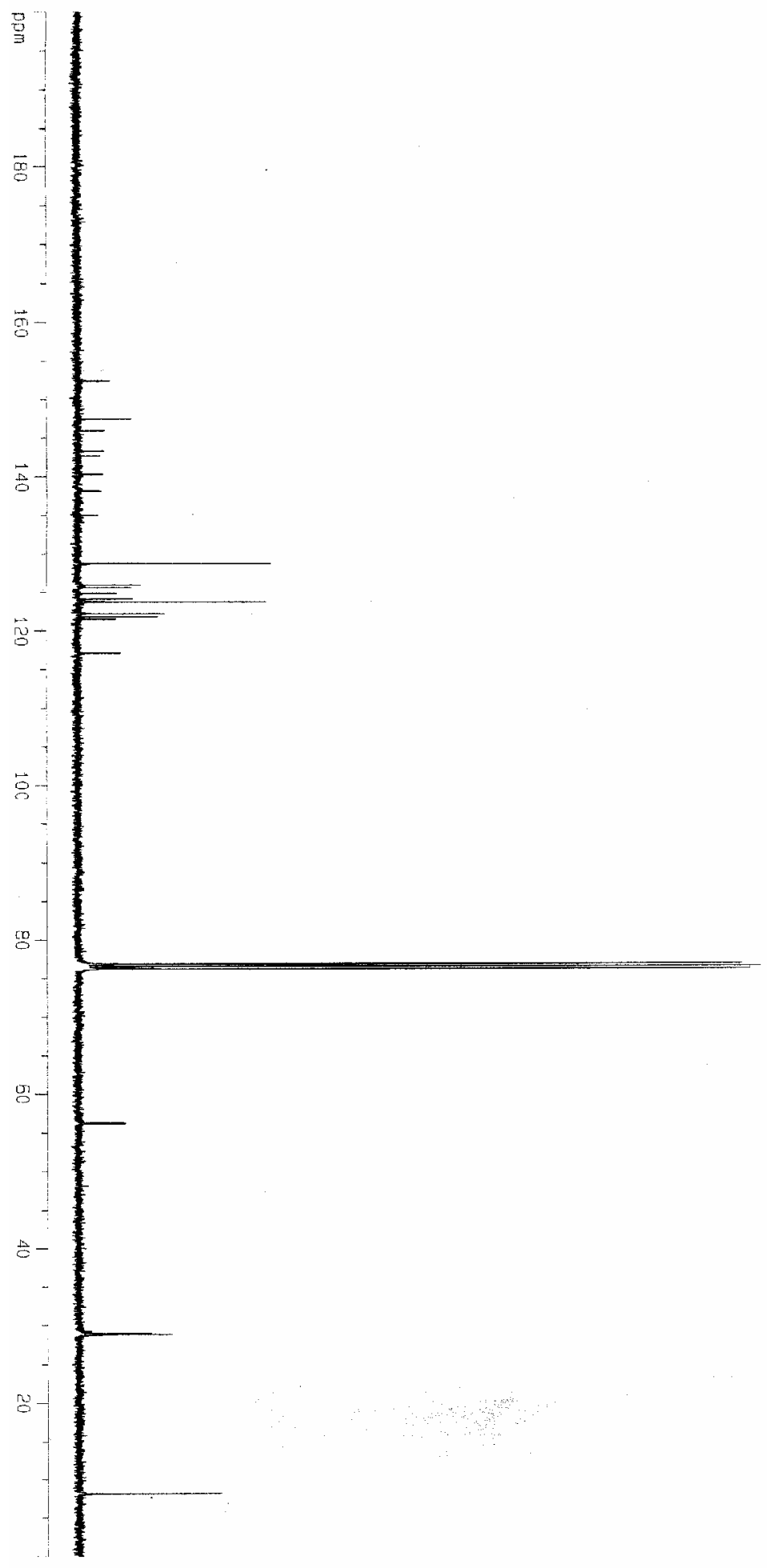

ofm
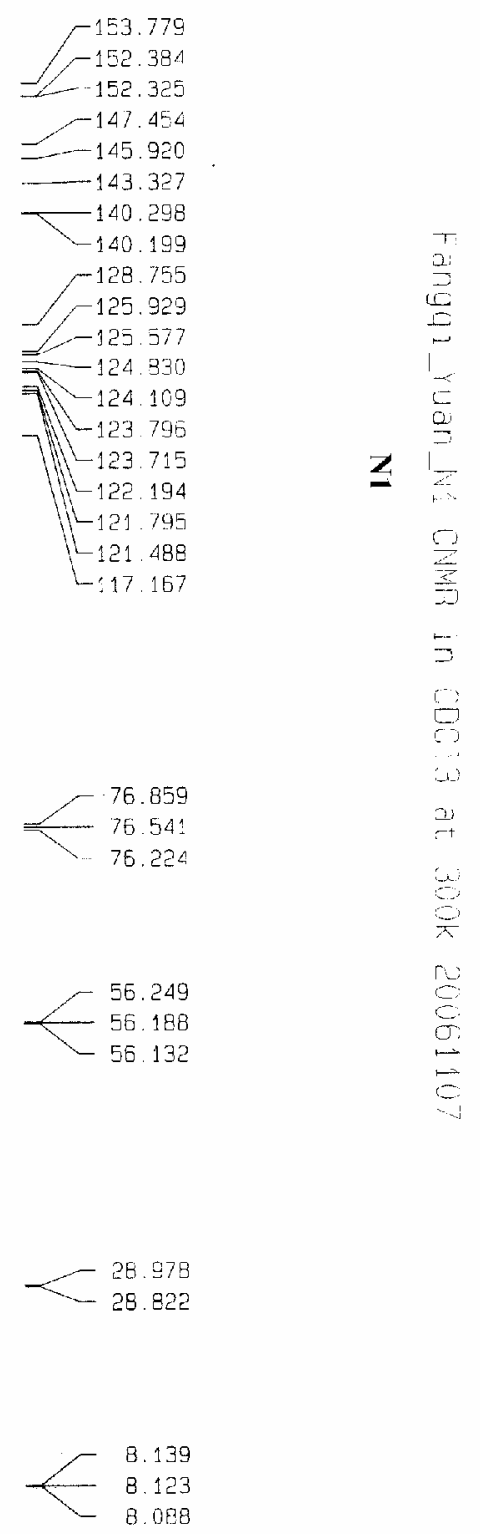

S50 

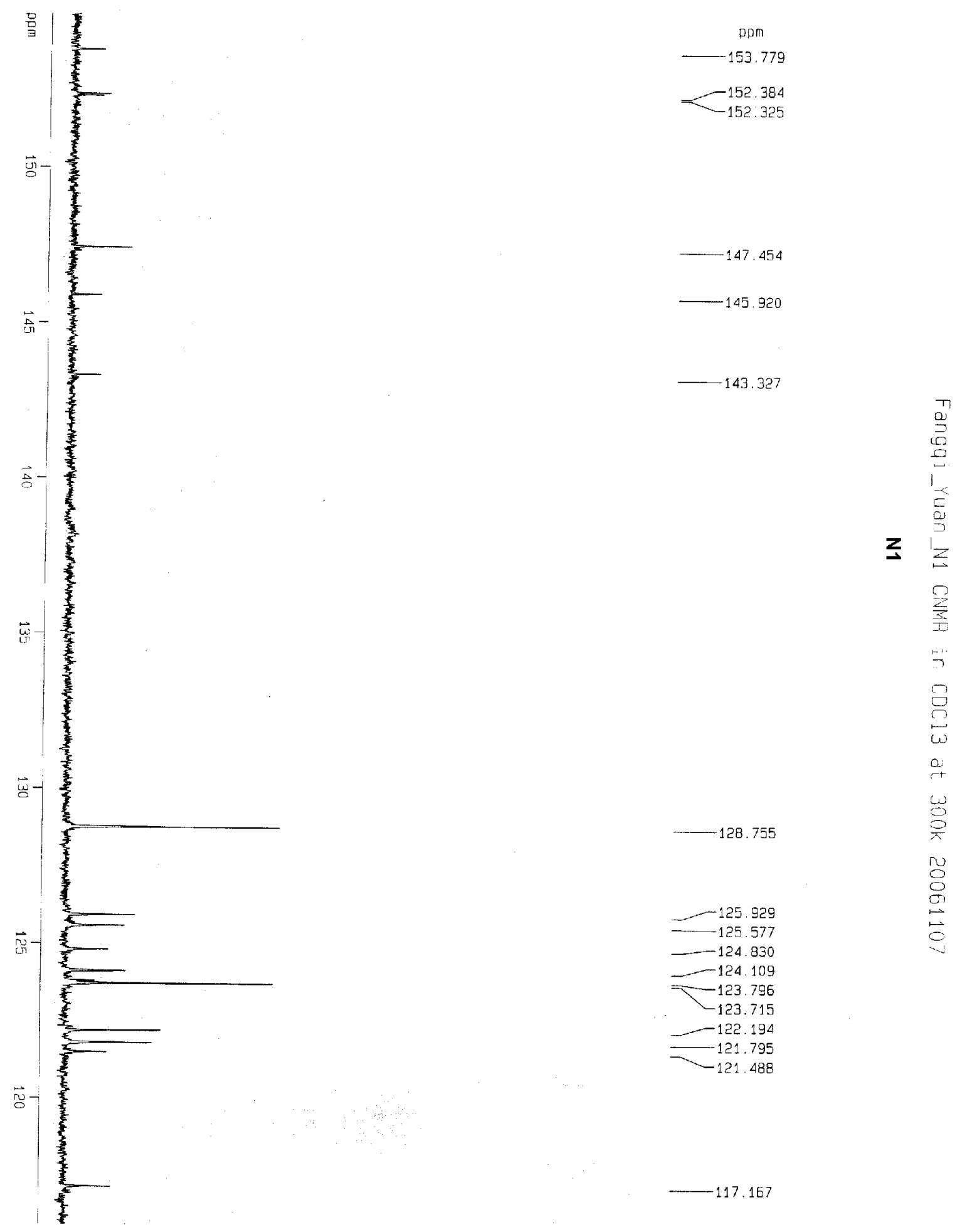


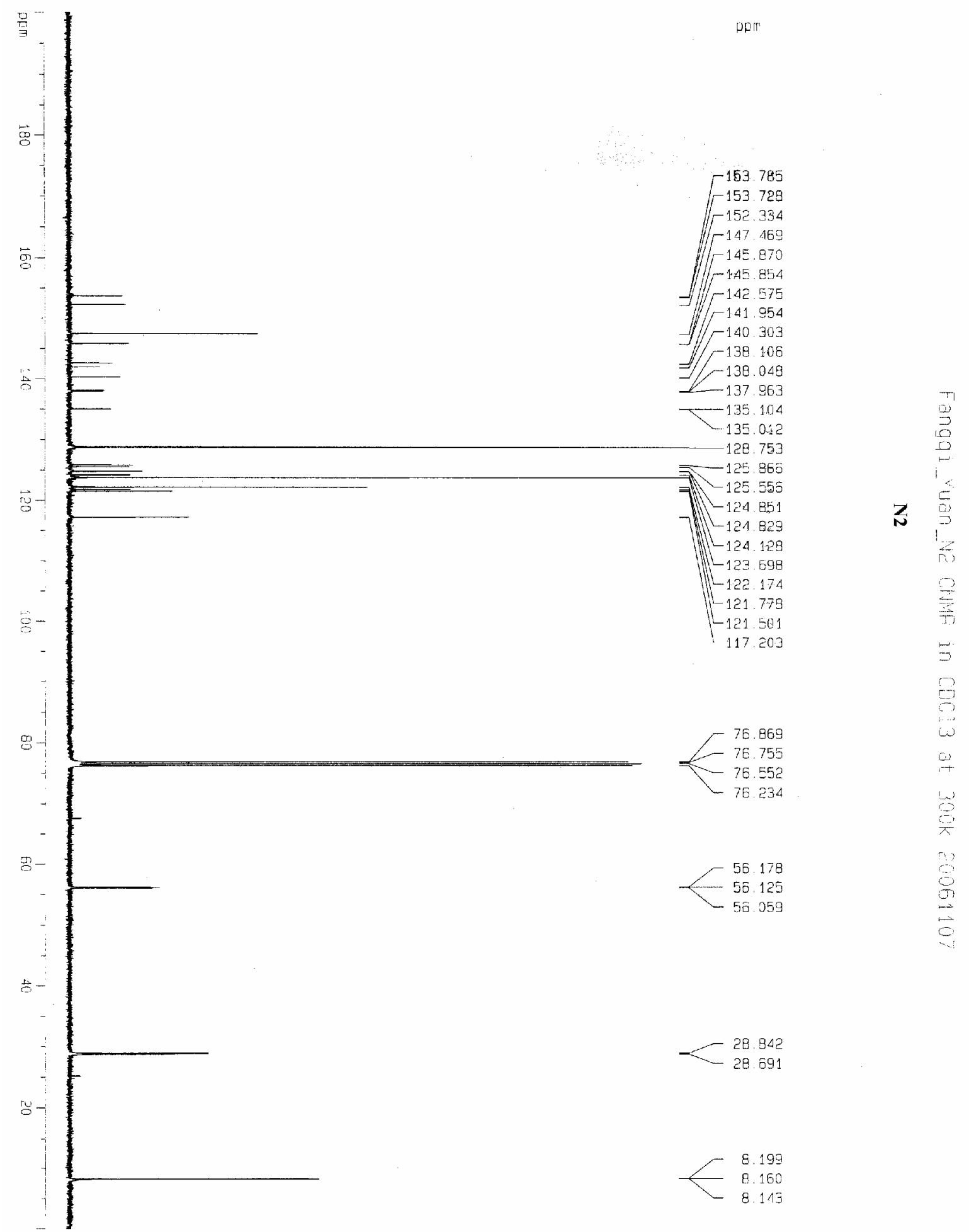



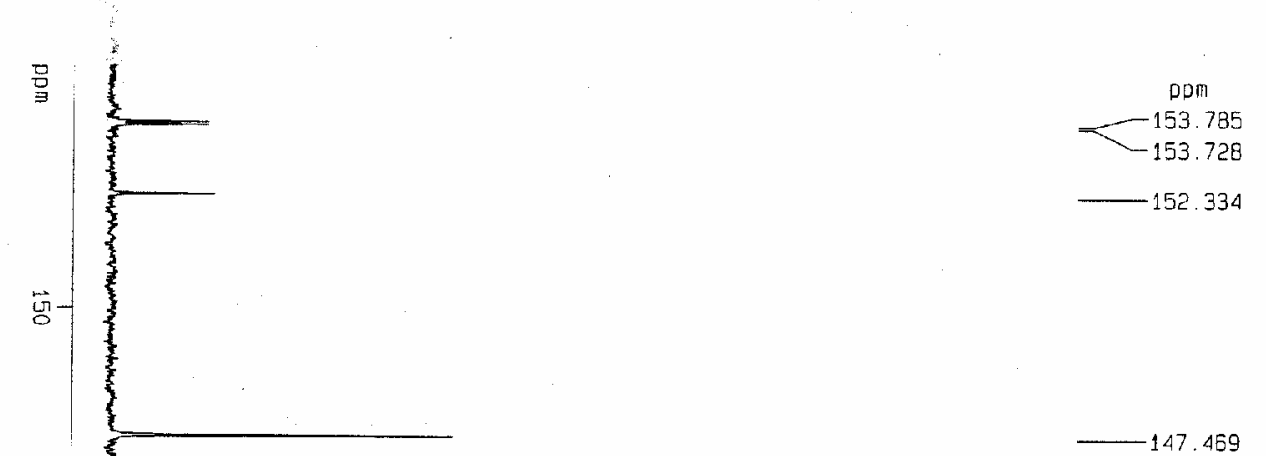

$-145.870$

.

홍-
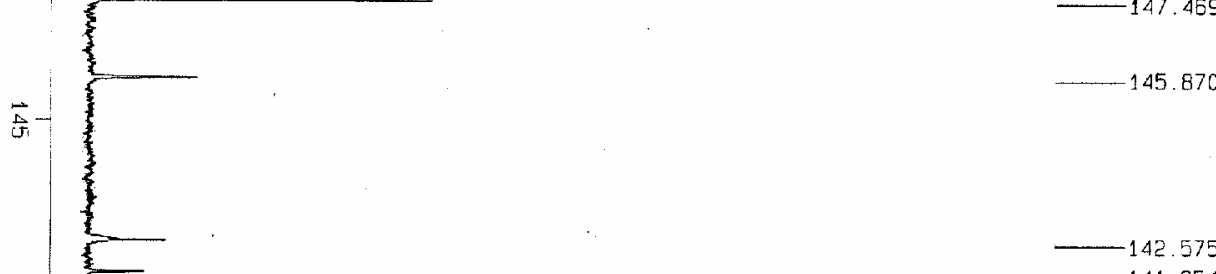

41.954

空-

$-140.303$

$-138.106$

138.048

$-137.963$

2

部

$-135.104$

$\frac{9}{3}$

$=-135.012$

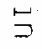

8

$\omega$

a

崖

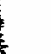

$-128.753$

巳

菏-

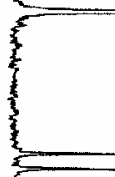

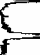

$-125.556$

$-124.851$

$-124.829$

$-124.128$

$-123.698$

$-122.174$

$=121.779$

$\sqrt{\frac{3}{3}}$

$-117.203$ 


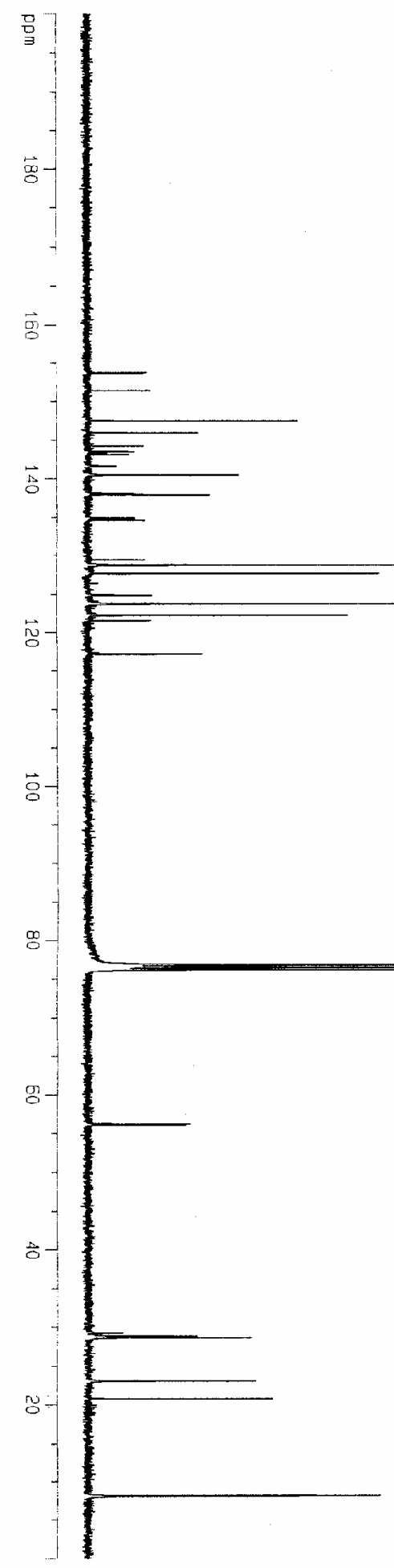

$-153.811$

$-153.669$

$-151.386$

$-147.464$

$-145.943$

-144.225

$\sqrt{143.428}$

II -140.446

1/ 140.446

] $/ \Gamma^{138.069}$

$-137.913$

$-134.955$

134.806

$-134.613$

Th 129.411

$7-128.739$

27.679

$-124.909$

$-124.860$

$-123.712$

L123.579

는.122.190

L121.538

L121.477

$L_{117.203}$

$-76.842$ 

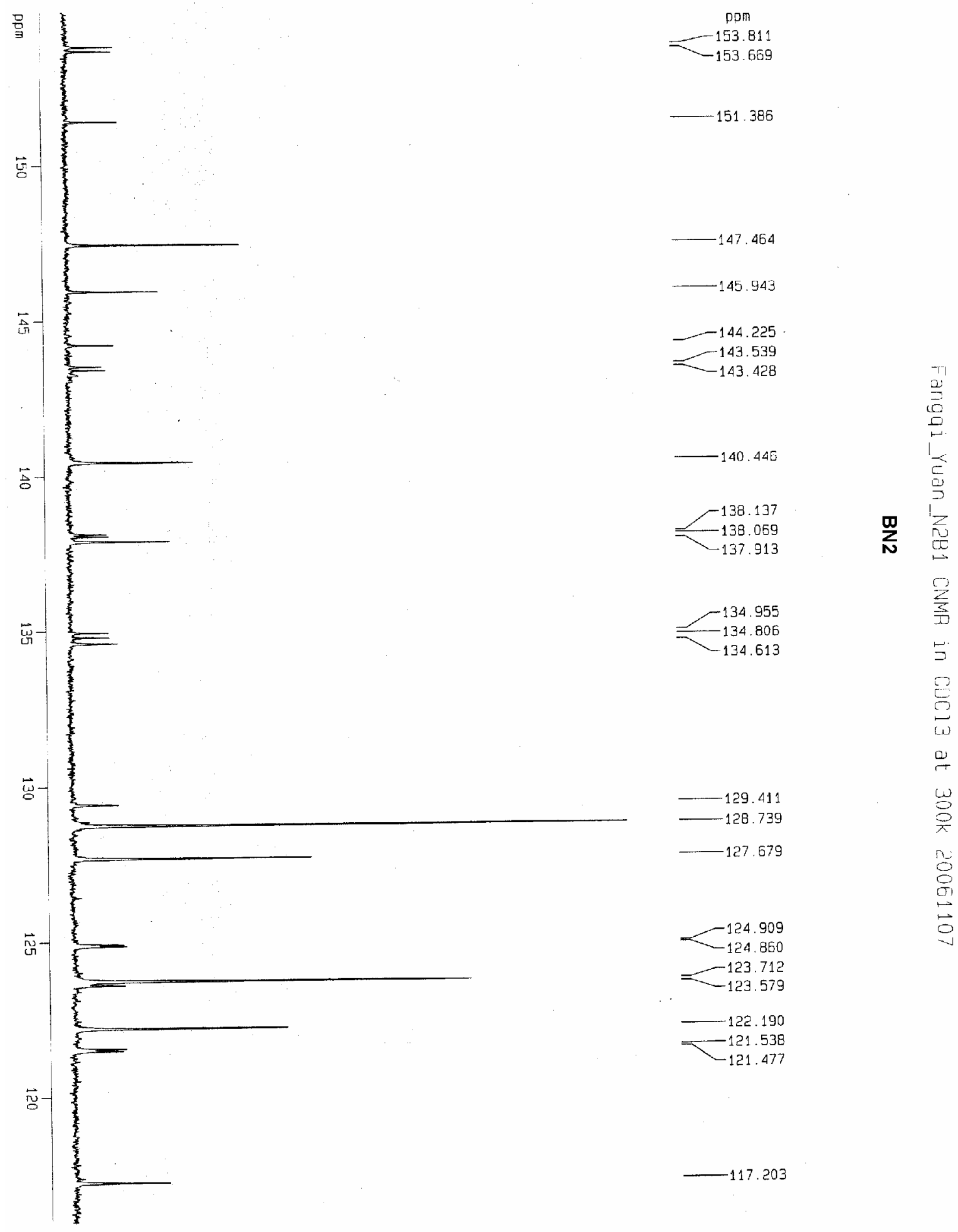

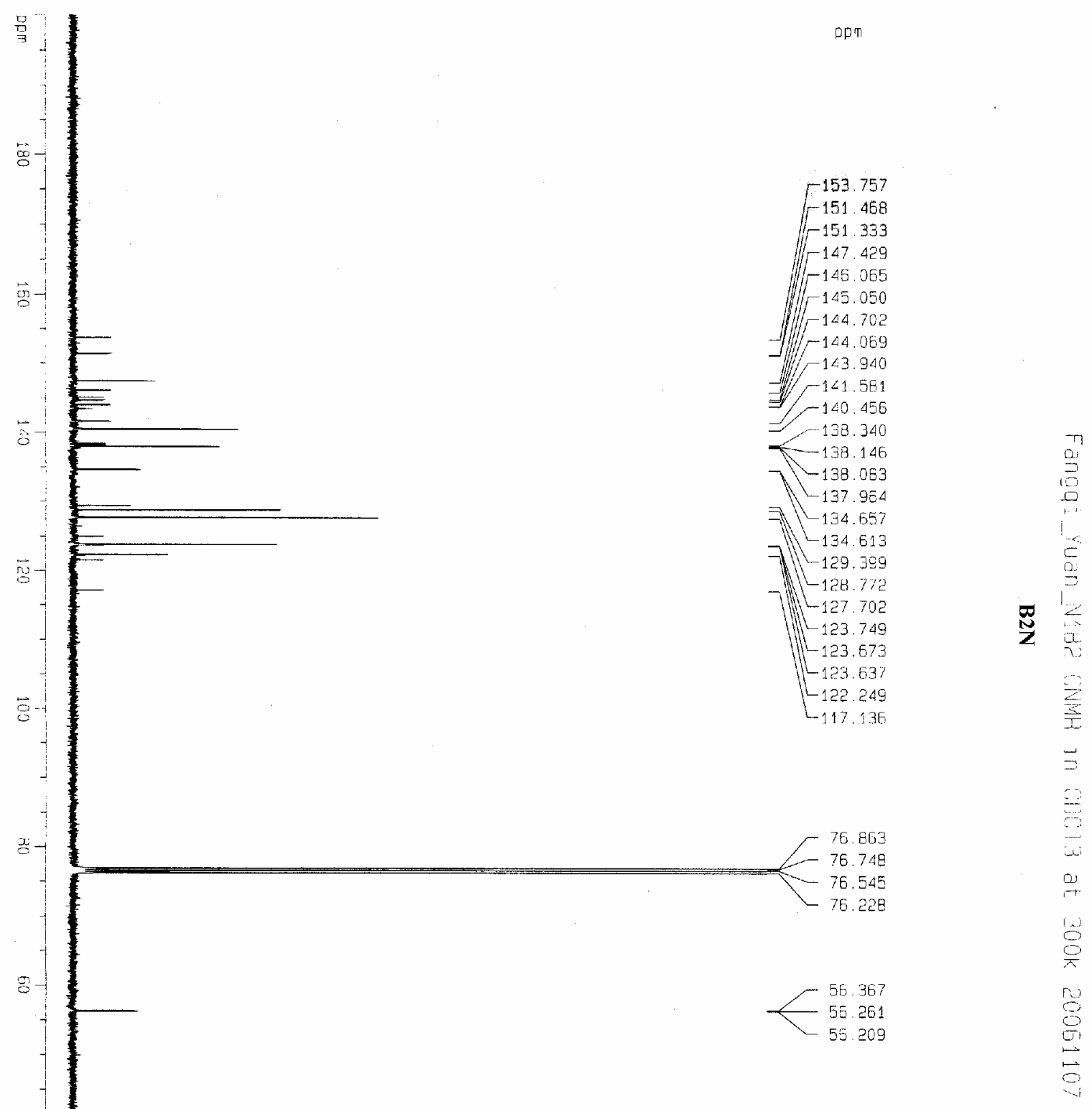

S56 

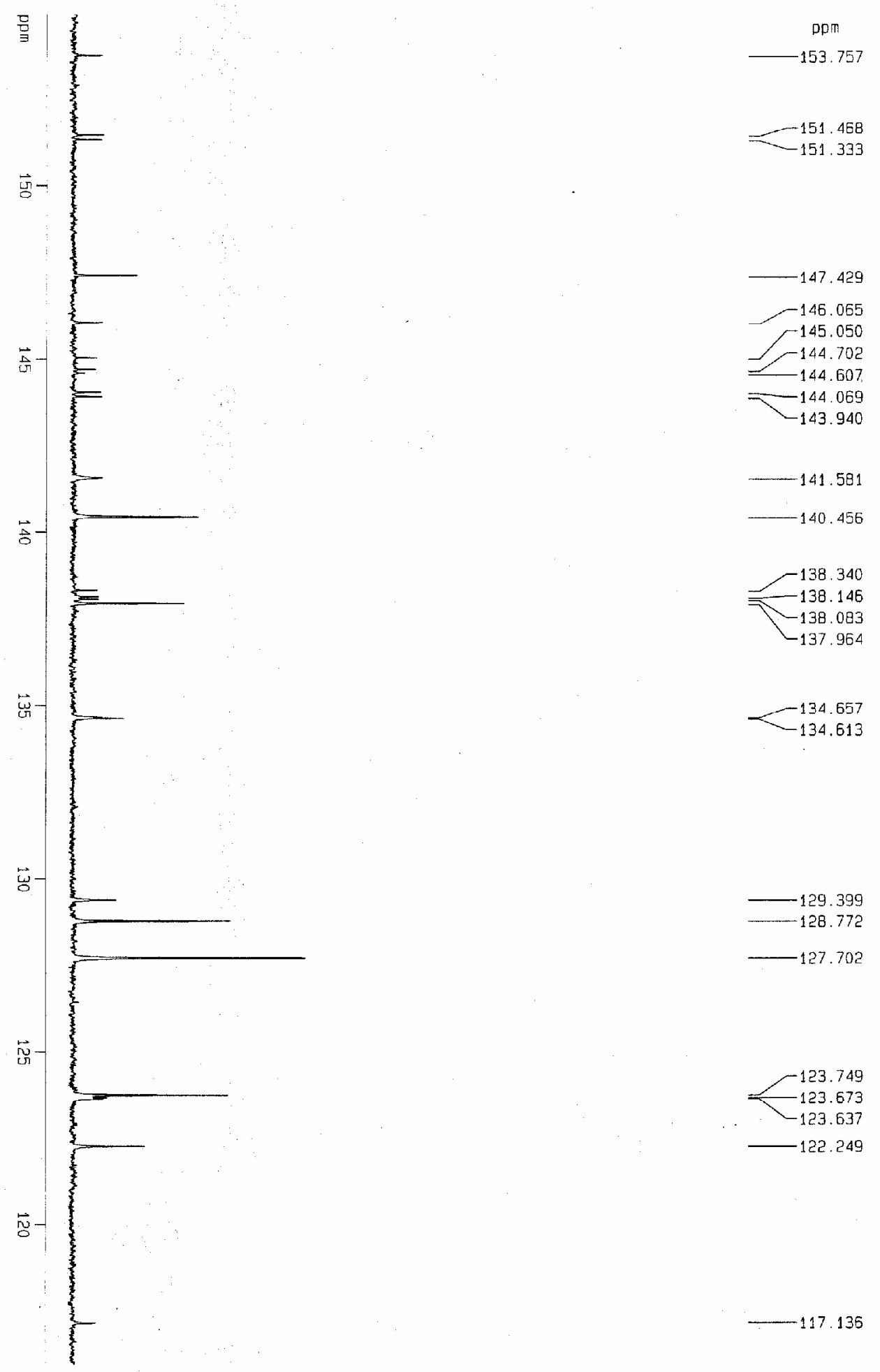

思壳

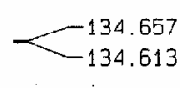

吝

5

号

$\stackrel{\square}{\sim}$

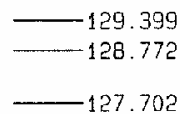

(5)

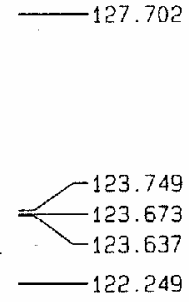

117.136
$-\quad$ 